\title{
ON THE RABINOWITZ FLOER HOMOLOGY OF TWISTED COTANGENT BUNDLES
}

\author{
WILL J. MERRY
}

\begin{abstract}
Let $(M, g)$ be a closed connected orientable Riemannian manifold of dimension $n \geq 2$. Let $\omega:=\omega_{0}+\pi^{*} \sigma$ denote a twisted symplectic form on $T^{*} M$, where $\sigma \in \Omega^{2}(M)$ is a closed 2-form and $\omega_{0}$ is the canonical symplectic structure $d p \wedge d q$ on $T^{*} M$. Suppose that $\sigma$ is weakly exact and its pullback to the universal cover $\widetilde{M}$ admits a bounded primitive. Let $H: T^{*} M \rightarrow \mathbb{R}$ be a Hamiltonian of the form $(q, p) \mapsto \frac{1}{2}|p|^{2}+U(q)$ for $U \in C^{\infty}(M, \mathbb{R})$. Let $\Sigma_{k}:=H^{-1}(k)$, and suppose that $k>$ $c(g, \sigma, U)$, where $c(g, \sigma, U)$ denotes the Mañé critical value. In this paper we compute the Rabinowitz Floer homology of such hypersurfaces.

Under the stronger condition that $k>c_{0}(g, \sigma, U)$, where $c_{0}(g, \sigma, U)$ denotes the strict Mañé critical value, Abbondandolo and Schwarz [4] recently computed the Rabinowitz Floer homology of such hypersurfaces, by means of a short exact sequence of chain complexes involving the Rabinowitz Floer chain complex and the Morse (co)chain complex associated to the free time action functional. We extend their results to the weaker case $k>c(g, \sigma, U)$, thus covering cases where $\sigma$ is not exact.

As a consequence, we deduce that the hypersurface $\Sigma_{k}$ is never (stably) displaceable for any $k>$ $c(g, \sigma, U)$. This removes the hypothesis of negative curvature in [20, Theorem 1.3] and thus answers a conjecture of Cieliebak, Frauenfelder and Paternain raised in [20]. Moreover, following [6 [5] we prove that for $k>c(g, \sigma, U)$, any $\psi \in \operatorname{Ham}_{c}\left(T^{*} M, \omega\right)$ has a leaf-wise intersection point in $\Sigma_{k}$, and that if in addition $\operatorname{dim} H_{*}\left(\Lambda M ; \mathbb{Z}_{2}\right)=\infty, \operatorname{dim} M \geq 2$, and the metric $g$ is chosen generically, then for a generic $\psi \in \operatorname{Ham}_{c}\left(T^{*} M, \omega\right)$ there exist infinitely many such leaf-wise intersection points.
\end{abstract}

\section{CONTENTS}

1. Introduction

2. Preliminaries

3. The free time action functional

4. The Rabinowitz action functional

5. The Abbondandolo-Schwarz short exact sequence

6. Non-displaceability and leaf-wise intersections above the critical value

References

\section{INTRODUCTION}

Let $(M, g)$ denote a closed connected orientable Riemannian manifold of dimension $n \geq 2$, with cotangent bundle $\pi: T^{*} M \rightarrow M$. Let $\omega_{0}=d \lambda_{0}$ denote the canonical symplectic form $d p \wedge d q$ on $T^{*} M$, where $\lambda_{0}$ is the Liouville 1 -form. Let $\widetilde{M}$ denote the universal cover of $M$. Let $\sigma \in \Omega^{2}(M)$ denote a closed weakly exact 2 -form, by this we mean that the pullback $\widetilde{\sigma} \in \Omega^{2}(\widetilde{M})$ is exact. We assume in addition that $\widetilde{\sigma}$ admits a bounded primitive. This means that there exists $\theta \in \Omega^{1}(\widetilde{M})$ with $d \theta=\widetilde{\sigma}$, and such that

$$
\|\theta\|_{\infty}:=\sup _{q \in \widetilde{M}}\left|\theta_{q}\right|<\infty,
$$

where $|\cdot|$ denotes the lift of the metric $g$ to $\widetilde{M}$. Let

$$
\omega:=\omega_{0}+\pi^{*} \sigma
$$


denote the twisted symplectic form determined by $\sigma$. We call the symplectic manifold $\left(T^{*} M, \omega\right)$ a twisted cotangent bundle.

Let $H_{g}: T^{*} M \rightarrow \mathbb{R}$ denote the standard "kinetic energy" Hamiltonian

$$
H_{g}(q, p):=\frac{1}{2}|p|^{2}
$$

Given a potential $U \in C^{\infty}(M, \mathbb{R})$, we study the autonomous Hamiltonian system defined by the convex mechanical Hamiltonian $H:=H_{g}+\pi^{*} U$. Let $X_{H}$ denote the symplectic gradient of $H$ with respect to the twisted symplectic form $\omega$, and let $\phi_{t}^{H}: T^{*} M \rightarrow T^{*} M$ denote the flow of $X_{H}$. The flow $\phi_{t}^{H}$ has a physical interpretation as the flow of a particle of unit mass and unit charge moving under the effect of an electric potential and a magnetic field, the former being represented by $U$ and the latter being represented by $\sigma$ (see for instance [10, 29]). The Lorentz force $Y: T M \rightarrow T M$ of $\sigma$ is the bundle map determined uniquely by

$$
\sigma_{q}(v, w)=\left\langle Y_{q}(v), w\right\rangle
$$

for $q \in M$ and $v, w \in T_{q} M$.

Given $k \in \mathbb{R}$, we let $\Sigma_{k}:=H^{-1}(k) \subseteq T^{*} M$. There are two particular "critical values" $c$ and $c_{0}$ of $k$, known as the Mañé critical values. They are such that the dynamics of the hypersurface $\Sigma_{k}$ differ dramatically depending on the relation of $k$ to these numbers. They satisfy $c<\infty$ if and only if $\widetilde{\sigma}$ admits a bounded primitive, and $c_{0}<\infty$ if and only if $\sigma$ is actually exact. If $\sigma$ is exact then whilst in a lot of cases one has $c=c_{0}$ (for instance, whenever $\pi_{1}(M)$ is amenable [26]), there may in general be a non-trivial interval $\left[c, c_{0}\right]$. In fact, this latter option happens quite frequently; see [20] for many explicit examples.

Our tool for investigating the hypersurfaces $\Sigma_{k}$ is Rabinowitz Floer homology, which was introduced by Cieliebak and Frauenfelder in [16], and then extended in various other directions by several other authors $([4,6,20,9,19,8,5,32])$. We refer the reader to the survey article [7] for a summary of the applications Rabinowitz Floer homology has generated so far. The present paper should be thought of as a supplement to [4]. Indeed, phrased in the language above, Theorem 2 of [4] deals with energy levels $k>c_{0}$ (in which case $\sigma$ is then necessarily exact). In this paper we study the weaker condition $k>c$. More precisely, we compute the Rabinowitz Floer homology (as defined in [20]) for any energy level $\Sigma_{k}$ with $k>c$. These computations are then used to answer a conjecture of Cieliebak, Frauenfelder and Paternain [20]; namely that for $k>c$ the hypersurface $\Sigma_{k}$ is never displaceable.

The starting point of Rabinowitz Floer homology is to work with a different action functional than the one normally used in Floer homology. This functional was originally introduced by Rabinowitz [45], and has the advantage that its critical points detect periodic orbits lying in a fixed energy level of the Hamiltonian. Let $\Lambda T^{*} M$ denote the free loop space of maps $x: S^{1} \rightarrow T^{*} M$ of Sobolev class $W^{1,2}$. Note that elements of $\Lambda T^{*} M$ are continuous. Given a free homotopy class $\alpha \in\left[S^{1}, M\right]$, let $\Lambda_{\alpha} T^{*} M$ denote the component of $\Lambda T^{*} M$ of loops whose projection to $M$ belong to $\alpha$. Fix a potential $U \in C^{\infty}(M, \mathbb{R})$ and put $H=H_{g}+\pi^{*} U$. Fix a regular energy value $k \in \mathbb{R}$ of $H$, and set $\Sigma_{k}:=H^{-1}(k)$. In order to introduce the Rabinowitz action functional, we begin by considering the 1-form $a_{H-k} \in \Omega^{1}\left(\Lambda T^{*} M \times \mathbb{R}\right)$ defined for $(x, \eta) \in \Lambda T^{*} M \times \mathbb{R}$ and $(\xi, b) \in T_{(x, \eta)}\left(\Lambda T^{*} M \times \mathbb{R}\right)$ by

$$
\left(a_{H-k}\right)_{(x, \eta)}(\xi, b):=\int_{S^{1}} \omega\left(\xi, \dot{x}-\eta X_{H}(x)\right) d t-b \int_{S^{1}}(H(x(t))-k) d t .
$$


The assumption that $\sigma$ is weakly exact implies the symplectic form $\omega$ is symplectically aspherical, that is, given any smooth function $f: S^{2} \rightarrow T^{*} M$ it holds that

$$
\int_{S^{2}} f^{*} \omega=0
$$

This implies that $a_{H-k}$ is exact on $\Lambda_{0} T^{*} M \times \mathbb{R}$, where $\Lambda_{0} T^{*} M \subseteq \Lambda T^{*} M$ denotes the component of $\Lambda T^{*} M$ of loops whose projection to $M$ is contractible. That is, there exists a function $A_{H-k}$ : $\Lambda_{0} T^{*} M \times \mathbb{R} \rightarrow \mathbb{R}$ called the Rabinowitz action functional with the property that

$$
\left.a_{H-k}\right|_{\Lambda_{0} T^{*} M \times \mathbb{R}}=d A_{H-k} .
$$

The functional $A_{H-k}$ is defined by

$$
A_{H-k}(x, \eta):=\int_{D^{2}} \bar{x}^{*} \omega-\eta \int_{S^{1}}(H(x(t))-k) d t,
$$

where $\bar{x} \in C^{0}\left(D^{2}, T^{*} M\right) \cap W^{1,2}\left(D^{2}, T^{*} M\right)$ is any map such that $\left.\bar{x}\right|_{\partial D^{2}}=x$. The symplectic asphericity condition implies that the value of $\int_{D^{2}} \bar{x}^{*} \omega$ is independent of the choice of filling disc $\bar{x}$. Our first observation is that the additional assumption that the lift $\widetilde{\sigma}$ of $\sigma$ to $\widetilde{M}$ admits a bounded primitive implies that the symplectic form $\omega$ is symplectically atoroidal, that is, given any smooth function $f: \mathbb{T}^{2} \rightarrow T^{*} M$ it holds that

$$
\int_{\mathbb{T}^{2}} f^{*} \omega=0
$$

(see Lemma2.3). In this case $a_{H-k}$ is actually exact on all of $\Lambda T^{*} M \times \mathbb{R}$. Indeed, for each $\alpha \in\left[S^{1}, M\right]$, fix a reference loop $x_{\alpha} \in \Lambda_{\alpha} T^{*} M$. Let $C:=S^{1} \times[0,1]$. Let $\bar{x} \in C^{0}\left(C, T^{*} M\right) \cap W^{1,2}\left(C, T^{*} M\right)$ denote any map such that $\bar{x}(\cdot, 0)=x$ and $\bar{x}(\cdot, 1)=x_{\alpha}$. Since $\omega$ is symplectically atoroidal, the value of $\int_{C} \bar{x}^{*} \omega$ is independent of the choice of $\bar{x}$. Thus we may define $A_{H-k}: \Lambda T^{*} M \times \mathbb{R} \rightarrow \mathbb{R}$ by

so that

$$
A_{H-k}(x, \eta):=\int_{C} \bar{x}^{*} \omega-\eta \int_{S^{1}}(H(x(t))-k) d t
$$

$$
a_{H-k}=d A_{H-k} \text {. }
$$

The critical points of $A_{H-k}$ are easily seen to satisfy:

$$
\begin{gathered}
\dot{x}=\eta X_{H}(x(t)) \quad \text { for all } t \in S^{1} ; \\
\int_{S^{1}}(H(x(t))-k) d t=0 .
\end{gathered}
$$

Since $H$ is invariant under its Hamiltonian flow, the second equation implies

$$
H(x(t))-k=0 \text { for all } t \in S^{1},
$$

that is,

$$
x\left(S^{1}\right) \subseteq \Sigma_{k} .
$$

Thus if Crit $\left(A_{H-k}\right)$ denotes the set of critical points of $A_{H-k}$, we can characterize $\operatorname{Crit}\left(A_{H-k}\right)$ by

$$
\begin{gathered}
\operatorname{Crit}\left(A_{H-k}\right)=\left\{(x, \eta) \in \Lambda T^{*} M \times \mathbb{R}: x \in C^{\infty}\left(S^{1}, T^{*} M\right)\right. \\
\left.\dot{x}(t)=\eta X_{H}^{\sigma}(x(t)), x\left(S^{1}\right) \subseteq \Sigma_{k}\right\} .
\end{gathered}
$$

For a generic choice of the metric $g$, the set Crit $\left(A_{H-k}\right)$ consists of a copy of the hypersurface $\Sigma_{k}$ (corresponding to the constant loops with $\eta=0$ ) and a discrete union of circles.

On the Lagrangian side we can play a similar game. Let $L_{g}: T M \rightarrow \mathbb{R}$ denote the standard "kinetic energy" Lagrangian defined by $L_{g}(q, v):=\frac{1}{2}|v|^{2}$, and given $U \in C^{\infty}(M, \mathbb{R})$ consider the Lagrangian $L:=L_{g}-\pi^{*} U$ (here we denote also by $\pi$ the footpoint map $T M \rightarrow M$ ). The Lagrangian $L$ is the 
Fenchel transform of the Hamiltonian $H=H_{g}+\pi^{*} U$ from above. Let $q_{\alpha}:=\pi \circ x_{\alpha}$, so that $q_{\alpha}$ is an element of the component $\Lambda_{\alpha} M$ corresponding to $\alpha$ of the free loop space $\Lambda M$. Given any $q \in \Lambda_{\alpha} M$, let $\bar{q} \in C^{0}(C, M) \cap W^{1,2}(C, M)$ denote any map such that $\bar{q}(\cdot, 0)=q$ and $\bar{q}(\cdot, 1)=q_{\alpha}$ (where $C=S^{1} \times[0,1]$ is as above). Then we define the free time action functional $S_{L+k}: \Lambda M \times \mathbb{R}^{+} \rightarrow \mathbb{R}$ by

$$
S_{L+k}(q, T):=T \int_{S^{1}}\left(L\left(q(t), \frac{\dot{q}(t)}{T}\right)+k\right) d t+\int_{C} \bar{q}^{*} \sigma
$$

If $\sigma$ is exact, this reduces to the definition of the standard free time action functional studied in [24, 22] (up to a constant).

If Crit $\left(S_{L+k}\right)$ denotes the set of critical points of $S_{L+k}$, then if $g$ is chosen genericaly the set $\operatorname{Crit}\left(S_{L+k}\right)$ consists of a discrete union of circles. If $L=L_{g}-\pi^{*} U$ and $H=H_{g}+\pi^{*} U$ then there is a close relationship between critical points of $S_{L+k}$ and critical points of $A_{H-k}$. Namely, each critical point $w=(q, T) \in \operatorname{Crit}\left(S_{L+k}\right)$ determines two critical points $Z^{ \pm}(w)=\left(x^{ \pm}, \pm T\right)$ of $A_{H-k}$. Here $x^{+}(t):=(q(t), \dot{q}(t))$ (where we have identified $T M$ with $T^{*} M$ via the Riemannian metric to see $\dot{q}(t)$ as an element of $\left.T_{q(t)}^{*} M\right)$ and $x^{-}(t):=x^{+}(-t)$. Then we have

$$
\left\{Z^{ \pm}(w): w \in \operatorname{Crit}\left(S_{L+k}\right)\right\}=\left\{(x, \eta) \in \operatorname{Crit}\left(A_{H-k}\right): \eta \neq 0\right\} .
$$

The "extra" critical points $(x, 0)$ of $A_{H-k}$ correspond to the so-called critical points at infinity of $S_{L+k}$, in the sense of Bahri [13]. Following [4], this motivates us to extend Crit $\left(S_{L+k}\right)$ to a new set

$$
\overline{\operatorname{Crit}}\left(S_{L+k}\right):=\operatorname{Crit}\left(S_{L+k}\right) \cup\{(q, 0): q \in M\} .
$$

For $k>c$, it turns out that one can do Morse theory with $S_{L+k}$. More precisely, after picking a Morse function $f: \overline{\operatorname{Crit}}\left(S_{L+k}\right) \rightarrow \mathbb{R}$, one can combine Frauenfelder's Morse-Bott homology with cascades [28, Appendix A] with Abbondandolo and Majer's infinite dimensional Morse theory [1] to construct a chain complex $C M_{*}\left(S_{L+k}, f\right)$ and a cochain complex $C M^{*}\left(S_{L+k}, f\right)$ whose associated Morse (co)homology $H M_{*}\left(S_{L+k}, f\right)$ and $H M^{*}\left(S_{L+k}, f\right)$ coincide with the singular (co)homology of $\Lambda M \times \mathbb{R}^{+}$.

The fact that there is such a strong relation between the critical points of $S_{L+k}$ and $A_{H-k}$ means that one is tempted to try and relate the Morse (co)homology of $S_{L+k}$ with the Rabinowitz Floer homology of $A_{H-k}$. This is precisely what Abbondandolo and Schwarz did, and in [4, Theorem 2] they construct (for $k>c_{0}$ ) a short exact sequence of chain complexes

$$
0 \rightarrow C M_{*}\left(S_{L+k}, f\right) \rightarrow R F_{*}\left(A_{H-k}, h\right) \rightarrow C M^{1-*}\left(S_{L+k},-f\right) \rightarrow 0 .
$$

Here $h: \operatorname{Crit}\left(A_{H-k}\right) \rightarrow \mathbb{R}$ denotes a Morse function on $\operatorname{Crit}\left(A_{H-k}\right)$ and $R F_{*}\left(A_{H-k}, h\right)$ denotes the Rabinowitz Floer chain complex of the pair $\left(A_{H-k}, h\right)$. We remark here that the Morse functions $f$ and $h$ must be related to each other in a fairly special way in order for such a short exact sequence to hold. Anyway, passing to the long exact sequence associated to this short exact of chain complexes and making the identification of the Morse (co)homology with the singular (co)homology of the loop space, this provides a way of computing the Rabinowitz Floer homology $R F H_{*}\left(A_{H-k}\right)$. Actually it must be said that this long exact sequence is a special case of a more general construction of Cieliebak, Frauenfelder and Oancea [19], which links Rabinowitz Floer homology with symplectic homology.

The aim of this paper is to show how the sequence (1.2) can be extended to the weaker case of $k>c$. In order to keep our exposition from being unnecessarily long, we only provide full details where there are substantial differences from [4]. Let us now summarize exactly what we do differently. On the Lagrangian side, more work must be done in order to define the Morse (co)complex; the key problem is to show that the Palais-Smale condition holds, which was shown in our previous work [38]. On the Hamiltonian side, we work directly with the Hamiltonians $H_{g}+\pi^{*} U$ that define the energy level $\Sigma_{k}$. 
This means that we cannot use the $L^{\infty}$ estimates on gradient flow lines of $A_{H-k}$ previously obtained in [16, 4, 20, 19]. Instead, we adapt the method of Abbondandolo and Schwarz in [3] to obtain our $L^{\infty}$ bounds. In fact, we are only able to obtain these $L^{\infty}$ bounds if we make an additional assumption on $\sigma$, namely that $\|\sigma\|_{\infty}$ is sufficiently small (cf. Remark 4.9, specifically (4.5)). However, a scaling argument, combined with invariance of the Rabinowitz Floer homology defined in [20] (see below) implies this is in fact no extra restriction at all.

A further difference is the question of grading; since we are working with the twisted symplectic form $\omega$, results such as Duistermaat's Morse index theorem [25] are not immediately available to us. Secondly the Hamiltonian $H$ is no longer a defining Hamiltonian (in the sense of [16]). This makes the computation of the Fredholm index of the operator obtained by linearizing the gradient of the Rabinowitz action functional along a flow line somewhat more complicated. Moreover unlike the corresponding situation in [4], the relationship between the Morse index of the fixed period action functional and the free time action functional is not so clear (cf. Theorem 3.5 and Remark 3.6). Full details of these index computations can be found in a supplementary paper joint with Gabriel P. Paternain [41].

Anyway, having proved such a short exact sequence (1.2), it is then clear that the Rabinowitz Floer homology $R F H_{*}\left(A_{H-k}\right)$ is non-zero whenever $k>c$. A key property of the Rabinowitz Floer homology $\operatorname{RFH}_{*}(\Sigma, V)$ constructed in [20], which is associated to a hypersurface $\Sigma$ of virtual restricted contact type in a geometrically bounded symplectically aspherical symplectic manifold $V$, is that if the hypersurface is displaceable then $R F H_{*}(\Sigma, V)$ vanishes. Assuming that our Rabinowitz Floer homology $R F H_{*}\left(A_{H-k}\right)$ is the same as the Rabinowitz Floer homology $R F H_{*}\left(\Sigma_{k}, T^{*} M\right)$ from [20], this would imply that $\Sigma_{k}$ is never displaceable for $k>c$. In Section 6 we prove that the two Rabinowitz Floer homologies are indeed isomorphic, and thus we arrive at the main result of this paper.

1.1. THEOREM. Let $(M, g)$ be a closed connected orientable Riemannian manifold and $\sigma \in \Omega^{2}(M)$ be a closed weakly exact 2 -form. Let $U \in C^{\infty}(M, \mathbb{R})$ and put $H:=H_{g}+\pi^{*} U$ and $\Sigma_{k}:=H^{-1}(k)$. Then if $k>c(g, \sigma, U)$ the Rabinowitz. Floer homology RF $H_{*}\left(\Sigma_{k}, T^{*} M\right)$ of [20] is defined and non-zero. In particular, $\Sigma_{k}$ is not displaceable.

1.2. REMARK. An alternative proof of Theorem 1.1] is given by Bae and Frauenfelder in [12]. Their idea is to show directly that the Rabinowitz Floer homology $R F H_{*}\left(\Sigma_{k}, T^{*} M ; \omega\right)$ as defined in [20] (where we temporarily add " $\omega$ " to the notation to indicate which symplectic form we are working with) is independent under certain perturbations of $\omega$. Using this, they prove that $R F H_{*}\left(\Sigma_{k}, T^{*} M ; \omega\right) \cong$ $R F H_{*}\left(\Sigma_{k}, T^{*} M ; \omega_{0}\right)$, from which they can deduce Theorem 1.1 from the corresponding results in [19, 4]. See also Remark 4.10 below.

1.3. REMARK. In fact, Theorem 1.1 proves that for $k>c$ the hypersurface $\Sigma_{k}$ is never stably displaceable. The concept of being stably displaceable is useful when the Euler characteristic $\chi(M)$ is non-zero. Indeed, when $\chi(M) \neq 0, \Sigma_{k}$ is never displaceable for topological reasons. However, it may be stably displaceable. To define stably displaceability, one considers the symplectic manifold $\left(T^{*} M \times T^{*} S^{1}, \omega \oplus \omega_{S^{1}}\right)$, where $\omega_{S^{1}}$ is the standard symplectic form on $T^{*} S^{1}$ (note that $\chi\left(M \times S^{1}\right)=0$ ). If $H=H_{g}+\pi^{*} U$ is a mechanical Hamiltonian on $T^{*} M$, consider the new Hamiltonian $\widehat{H}: T^{*}\left(M \times S^{1}\right) \rightarrow \mathbb{R}$ defined by

$$
\begin{aligned}
\widehat{H}\left(q, p, t, p_{t}\right): & =H(q, p)+\frac{1}{2}\left|p_{t}\right|^{2} \quad p \in T_{q}^{*} M, p_{t} \in T_{t}^{*} S^{1} \\
& =\frac{1}{2}|p|^{2}+U(q)+\frac{1}{2}\left|p_{t}\right|^{2} .
\end{aligned}
$$

\footnotetext{
${ }^{1}$ The hypersurface $\Sigma_{k}$ is virtually contact if $k>c$ [20] Lemma 5.1], so $R F H_{*}\left(\Sigma_{k}, T^{*} M\right)$ as defined in [20] is well defined.
} 
Let $\widehat{\Sigma}_{k}:=\widehat{H}^{-1}(k)$. Then by definition $\Sigma_{k}$ is stably displaceable if $\widehat{\Sigma}_{k}$ is displaceable. In order to see why our theorem implies that $\Sigma_{k}$ is never stably displaceable for $k>c$, one uses the following observation of Macarini and Paternain [36. Lemma 2.2]: if c denotes the Mañé critical value of $H$ and $\widehat{c}$ denotes the Mañé critical value of $\widehat{H}$ then $\widehat{2} \widehat{c}=c$. Thus if $k>c$ then also $k>\widehat{c}$, and so applying Theorem [1.1 to $\widehat{\Sigma}_{k}$ we see that $\widehat{\Sigma}_{k}$ is not displaceable, and hence $\Sigma_{k}$ is not stably displaceable.

1.4. RemARK. Strictly speaking, the Rabinowitz Floer homology $R F H_{*}\left(\Sigma_{k}, T^{*} M\right)$ as defined in [20] is only defined for contractible loops, as the observation that the twisted symplectic form $\omega$ is symplectically atoroidal was not used in that paper. However, if one uses this observation, the construction in [20] allows one to define $R F H_{*}\left(\Sigma_{k}, T^{*} M\right)$ for any free homotopy class of loops. The proof given in Section 6 shows that our $R_{F} H_{*}\left(A_{H-k}\right)$ agrees with this Rabinowitz Floer homology $R F H_{*}\left(\Sigma_{k}, T^{*} M\right)$ (in any free homotopy class). The reader however may prefer to read Section 6 as if we were only working with contractible loops (which is sufficient for the non-displaceability application we have in mind).

1.5. REMARK. In [39] we compute the Lagrangian Rabinowitz Floer homology of the hypersurface $\Sigma_{k}$, where for the Lagrangian submanifolds of $T^{*} M$ involved we take two cotangent fibres $T_{q_{0}}^{*} M$ and $T_{q_{1}}^{*} M$ (where possibly $q_{0}=q_{1}$ ). We show that a similar short exact sequence to (1.2) exists between the Lagrangian Rabinowitz Floer homology

$$
R F H_{*}\left(\Sigma_{k}, T_{q_{0}}^{*} M, T_{q_{1}}^{*} M, T^{*} M\right)
$$

and the Morse (co)homology of the free time action functional, this time defined on the path space $\Omega\left(M, q_{0}, q_{1}\right)$ of paths in $M$ from $q_{0}$ to $q_{1}$.

Having proved that for $k>c$ the Rabinowitz Floer homology $R F H_{*}\left(\Sigma_{k}, T^{*} M\right)$ is non-zero, one can prove a much stronger statement than non-displaceability, which we will now explain. Let $\operatorname{Ham}_{c}\left(T^{*} M, \omega\right)$ denote the set of compactly supported Hamiltonian diffeomorphisms of the symplectic manifold $\left(T^{*} M, \omega\right)$, that is

$$
\operatorname{Ham}_{c}\left(T^{*} M, \omega\right):=\left\{\phi_{1}^{F}: F \in C_{c}^{\infty}\left(S^{1} \times T^{*} M, \mathbb{R}\right)\right\},
$$

where $\phi_{t}^{F}$ is the flow of $X_{F}$; the latter being the time-dependent symplectic gradient of $F$ with respect to $\omega$.

Fix $H=H_{g}+\pi^{*} U$ and put $\Sigma_{k}:=H^{-1}(k)$. Given $x \in \Sigma_{k}$, let us write $\mathcal{L}_{x}$ for the leaf of the characteristic foliation of $\Sigma_{k}$ passing through $x$, that is,

$$
\mathcal{L}_{x}:=\left\{\phi_{t}^{H}(x): t \in \mathbb{R}\right\},
$$

so that $\Sigma_{k}$ is foliated by the leaves $\left\{\mathcal{L}_{x}: x \in \Sigma_{k}\right\}$. Given $\psi \in \operatorname{Ham}_{c}\left(T^{*} M, \omega\right)$, a point $x \in \Sigma_{k}$ is called a leaf-wise intersection point for $\psi$ if $\psi(x) \in \mathcal{L}_{x}$. By following through the proofs in [6, 5] we can prove the following result.

1.6. THEOREM. Let $(M, g)$ be a closed connected orientable Riemannian manifold and $\sigma \in \Omega^{2}(M)$ be a closed weakly exact 2-form. Let $U \in C^{\infty}(M, \mathbb{R})$ and put $H:=H_{g}+\pi^{*} U$. Choose $k>c(g, \sigma, U)$ and put $\Sigma_{k}:=H^{-1}(k)$. Then for any $\psi \in \operatorname{Ham}_{c}\left(T^{*} M, \omega\right)$ there exists a leaf-wise intersection point for $\psi$ in $\Sigma_{k}$. Moreover, if $\operatorname{dim} H_{*}\left(\Lambda M ; \mathbb{Z}_{2}\right)=\infty$ and $g$ is chosen generically, then for a generic $\psi \in \operatorname{Ham}_{c}\left(T^{*} M, \omega\right)$ there exist infinitely many leaf-wise intersection points for $\psi$ in $\Sigma_{k}$.

We conclude this introduction with a remark about how the results of this paper extend to more general Hamiltonian systems.

\footnotetext{
${ }^{2}$ Actually [36 Lemma 2.2] works with the strict Mañé critical values $c_{0}$ and $\widehat{c}_{0}$, but exactly the same proof (working on $\tilde{M}$ instead of $M$ ) shows that $c=\widehat{c}$.
} 
1.7. REMARK. In fact, all of the results in the present paper are valid under more general hypotheses, as we now explain. Recall that an autonomous Hamiltonian $K \in C^{\infty}\left(T^{*} M, \mathbb{R}\right)$ is called Tonelli if $K$ is fibrewise strictly convex and superlinear. In other words, the second differential $d^{2}\left(\left.K\right|_{T_{q}^{*} M}\right)$ of $K$ restricted to each tangent space $T_{q}^{*} M$ is positive definite, and

$$
\lim _{|p| \rightarrow \infty} \frac{K(q, p)}{|p|}=\infty
$$

uniformly for $q \in M$. As with mechanical Hamiltonians, given a Tonelli Hamiltonian $K$ and a weakly exact 2-form $\sigma$, there exists a critical value $c(K, \sigma)$ called the Mañé critical value. As before, $c(K, \sigma)<\infty$ if and only if $\widetilde{\sigma}$ admits a bounded primitive. Let us say that a closed connected orientable hypersurface $\Sigma \subseteq T^{*} M$ is a Mañé supercritical hypersurface if there exists a Tonelli Hamiltonian $K$ such that $\Sigma=K^{-1}(k)$ for some $k>c(K, \sigma)$.

Both Theorem 1.1 and Theorem 1.6 extend to Mañé supercritical hypersurfaces. Namely: the Rabinowitz Floer homology of any Mañé supercritical hypersurface is defined and non-zero. In particular, Mañé supercritical hypersurfaces are never displaceable. Secondly, given any Mañé supercritical hypersurface $\Sigma$ and any $\psi \in \operatorname{Ham}_{c}\left(T^{*} M, \omega\right)$ there exists a leaf-wise intersection point for $\psi$ in $\Sigma$. Moreover, if $\operatorname{dim} H_{*}\left(\Lambda M ; \mathbb{Z}_{2}\right)=\infty$ and $\Sigma$ is non-degenerate (which holds generically), then for a generic $\psi \in \operatorname{Ham}_{c}\left(T^{*} M, \omega\right)$ there exist infinitely many leaf-wise intersection points for $\psi$ in $\Sigma$.

More details about these results can be found in [40].

Acknowledgments. I would like to thank my Ph.D. adviser Gabriel P. Paternain for many helpful discussions. I am also extremely grateful to Alberto Abbondandolo, Peter Albers and Urs Frauenfelder for several stimulating remarks and insightful suggestions, and for pointing out errors in previous drafts of this work.

\section{PRELIMINARIES}

We denote by $\overline{\mathbb{R}}$ the extended real line $\overline{\mathbb{R}}:=\mathbb{R} \cup\{ \pm \infty\}$, with the differentiable structure induced by the bijection $[-\pi / 2, \pi / 2] \rightarrow \overline{\mathbb{R}}$ given by

$$
s \mapsto \begin{cases}\tan s & s \in(-\pi / 2, \pi / 2) \\ \pm \infty & s= \pm \pi / 2 .\end{cases}
$$

We denote by $\mathbb{R}^{+}, \mathbb{R}_{0}^{+}$the spaces $(0, \infty)$ and $[0, \infty)$, with similar conventions for $\mathbb{R}^{-}, \mathbb{R}_{0}^{-}$. We will often identify $S^{1}$ with $\mathbb{R} / \mathbb{Z}$. We adopt throughout the convenient convention that any manifold asserted to have negative dimension is in fact, empty. Another convention we use throughout is: given a function $f(s, t)$ of two variables $s, t$ (usually $(s, t) \in \mathbb{R} \times \mathbb{T}$ ) we let $f^{\prime}:=\partial_{s} f$ and $\dot{f}:=\partial_{t} f$. Throughout the paper we will freely and ambiguously use the isometry $T M \cong T^{*} M, v \mapsto\langle v, \cdot\rangle$, determined by the Riemannian metric $g$, to identify points in $T_{q} M$ with points in $T_{q}^{*} M$.

\section{All the sign conventions used in this paper match those of [4].}

\subsection{The loop spaces.}

Let $W^{1,2}([0,1], M)$ denote the Hilbert manifold of paths $q:[0,1] \rightarrow M$ of Sobolev class $W^{1,2}$. Note that elements of $W^{1,2}([0,1], M)$ are continuous. Let $\Lambda M$ denote the submanifold consisting of loops $q: S^{1} \rightarrow M$ of Sobolev class $W^{1,2}$. Note that $\Lambda M$ is homotopy equivalent to both $C^{0}\left(S^{1}, M\right)$ and $C^{\infty}\left(S^{1}, M\right)$. We can identify $T_{q} \Lambda M$ with $W^{1,2}\left(S^{1}, q^{*} T M\right)$, that is, the sections $\zeta: S^{1} \rightarrow q^{*} T M$ of class $W^{1,2}$. Given a free homotopy class $\alpha \in\left[S^{1}, M\right]$, let $\Lambda_{\alpha} M \subseteq \Lambda M$ denote the connected component of $\Lambda M$ consisting of the loops $q \in \Lambda M$ belonging to the free homotopy class $\alpha$. Given $\alpha \in\left[S^{1}, M\right]$, we write $-\alpha$ for the free homotopy class that contains the loops $q^{-}(t):=q(-t)$ for $q \in \Lambda_{\alpha} M$. 
Similarly we let $W^{1,2}\left([0,1], T^{*} M\right)$ denote the Hilbert manifold of paths $x:[0,1] \rightarrow T^{*} M$ of Sobolev class $W^{1,2}$. Note that elements of $W^{1,2}\left([0,1], T^{*} M\right)$ are continuous. Denote by $\Lambda T^{*} M$ the submanifold of loops $x: S^{1} \rightarrow T^{*} M$ of Sobolev class $W^{1,2}$. Note that $\Lambda T^{*} M$ is homotopy equivalent to both $C^{0}\left(S^{1}, T^{*} M\right)$ and $C^{\infty}\left(S^{1}, T^{*} M\right)$. The tangent space $T_{x} \Lambda T^{*} M$ can be identified with $W^{1,2}\left(S^{1}, x^{*} T^{*} M\right)$, that is, the sections $\xi: S^{1} \rightarrow x^{*} T T^{*} M$ of class $W^{1,2}$. Given $\alpha \in\left[S^{1}, M\right]$, we let $\Lambda_{\alpha} T M$ denote the set of loops $x \in \Lambda T^{*} M$ whose projection $\pi \circ x$ lies in $\Lambda_{\alpha} M$.

Using the metric $g=\langle\cdot, \cdot\rangle$ on $M$ we obtain a metric $\langle\langle\cdot, \cdot\rangle\rangle_{g}$ on $\Lambda M \times \mathbb{R}^{+}$via

$$
\langle\langle(\zeta, b),(\vartheta, e)\rangle\rangle_{g}:=\int_{S^{1}}\left\{\langle\zeta, \vartheta\rangle+\left\langle\nabla_{t} \zeta, \nabla_{t} \vartheta\right\rangle\right\} d t+b e,
$$

where $\nabla$ denotes the Levi-Civita connection of $(M, g)$.

Let $\mathcal{J}$ denote the space of 1-periodic almost complex structures on $T^{*} M$ with finite $L^{\infty}$ norm, and equip $\mathcal{J}$ with the $L^{\infty}$ norm. The metric $g$ determines a special autonomous almost complex structure $J_{g} \in \mathcal{J}$ called the metric almost complex structure. To define the metric almost complex structure, we first recall that the metric $g$ determines a direct summand $T^{h} T^{*} M$ of the vertical tangent bundle $T^{v} T^{*} M:=$ ker $d \pi$, together with an isomorphism

$$
T_{x} T^{*} M=T_{x}^{h} T^{*} M \oplus T_{x}^{v} T^{*} M \cong T_{q} M \oplus T_{q}^{*} M, \quad x=(q, p) \in T^{*} M .
$$

The metric almost complex structure $J_{g}$ is defined in terms of this splitting by

$$
J_{g}:=\left(\begin{array}{cc}
0 & -\mathbb{1} \\
\mathbb{1} & 0
\end{array}\right) .
$$

Let $\mathcal{J}(\omega)$ denote the space of 1-periodic almost complex structures on $T^{*} M$ that are $\omega$-compatible and satisfy $\|J\|_{\infty}<\infty$. In general $J_{g} \notin \mathcal{J}(\omega)$. However if $B_{r}\left(J_{g}\right)$ denotes the open ball of radius $r>0$ about the metric almost complex structure $J_{g}$ in $\mathcal{J}$ then [34, Proposition 4.1] implies that there exists a constant $\varepsilon_{0}=\varepsilon_{0}(g)>0$ (which depends continuously on $g$ ) such that if $r>\varepsilon_{0}\|\sigma\|_{\infty}$ then

$$
\mathcal{J}(\omega) \cap B_{r}\left(J_{g}\right) \neq \emptyset \text { if } r>\varepsilon_{0}\|\sigma\|_{\infty} .
$$

This will be important in the proof of Theorem 4.14, see also Remark4.9 Given $J \in \mathcal{J}(\omega)$ we obtain a 1-periodic Riemannian metric $\langle\cdot, \cdot\rangle_{J}=\omega(J \cdot, \cdot)$ on $T^{*} M$. We will write $\langle\langle\cdot, \cdot\rangle\rangle_{J}$ for the $L^{2}$-metric on $\Lambda T^{*} M \times \mathbb{R}$ defined by

$$
\langle\langle(\xi, b),(\rho, e)\rangle\rangle_{J}:=\int_{S^{1}}\langle\xi, \rho\rangle_{J}+b e .
$$

Finally let us remark that the first Chern class $c_{1}\left(T^{*} M, J\right)=0$ for any $J \in \mathcal{J}(\omega)$; one way to see this is that the twisted symplectic manifold $\left(T^{*} M, \omega\right)$ admits a Lagrangian distribution $T^{v} T^{*} M$ (see for example [47, Example 2.10]).

\subsection{Mañé's critical values.}

We now recall the definition of the two critical values $c$ and $c_{0}$ associated to the triple $(g, \sigma, U)$, introduced by Mañé in [35], which play a decisive role in all that follows. General references for the results stated below are [23, Proposition 2-1.1] or [15, Appendix A].

Fix $U \in C^{\infty}(M, \mathbb{R})$, and let $H: T^{*} M \rightarrow \mathbb{R}$ be defined by $H:=H_{g}+\pi^{*} U$. Given $k \in \mathbb{R}$, let $\Sigma_{k}:=H^{-1}(k)$. Define the Mañé critical value associated to the metric $g$, the weakly exact 2 -form $\sigma$

\footnotetext{
${ }^{3}$ In fact, [34, Proposition 4.1] shows that for $r>\varepsilon_{0}\|\sigma\|_{\infty}$ we may even find geometrically bounded almost complex structures in $\mathcal{J}(\omega) \cap B_{r}\left(J_{g}\right)$; see Remark6.1
} 
and the potential $U$ by:

$$
c=c(g, \sigma, U):=\inf _{\theta} \sup _{q \in \widetilde{M}} \widetilde{H}\left(q, \theta_{q}\right),
$$

where the infimum is taken over all 1-forms $\theta$ on $\widetilde{M}$ with $d \theta=\widetilde{\sigma}$, and $\widetilde{H}$ is the lift of $H$ to $T^{*} \widetilde{M}$. Thus $c(g, \sigma, U)<\infty$ if and only if $\widetilde{\sigma}$ admits a bounded primitive.

If $\sigma$ is exact, define the strict Mañé critical value $c_{0}=c_{0}(g, \sigma, U)$ by

$$
c_{0}=c_{0}(g, \sigma, U):=\inf _{\theta} \sup _{q \in M} H\left(q, \theta_{q}\right)<\infty
$$

that is, the same definition only working directly on $T^{*} M$ rather than lifting to $T^{*} \widetilde{M}$. If $\sigma$ is not exact, set $c_{0}(g, \sigma, U):=\infty$. Note in all cases we have

$$
c \leq c_{0} \leq \infty .
$$

The critical value can also be defined in Lagrangian terms. Let $L:=L_{g}-\pi^{*} U$ denote the Fenchel dual Lagrangian to $H$, and let $\widetilde{L}$ denote the lift of $L$ to $T \widetilde{M}$. Fix a primitive $\theta$ of $\widetilde{\sigma}$, and think of $\theta$ as a smooth function on $T \widetilde{M}$. Now consider the Lagrangian $\widetilde{L}+\theta$. The action $\mathbb{A}_{\widetilde{L}+\theta}(\gamma)$ on an absolutely continuous curve $\gamma:[a, b] \rightarrow \widetilde{M}$ is defined by

$$
\mathbb{A}_{\widetilde{L}+\theta}(\gamma):=\int_{a}^{b}(\widetilde{L}+\theta)(\gamma(t), \dot{\gamma}(t)) d t=\int_{a}^{b} \widetilde{L}(\gamma(t), \dot{\gamma}(t))+\theta_{\gamma(t)}(\dot{\gamma}(t)) d t,
$$

and an alternative definition of $c$ is the following:

$$
c:=\inf \left\{k \in \mathbb{R}: \mathbb{A}_{\widetilde{L}+\theta+k}(\gamma) \geq 0 \forall \text { a.c. closed curves defined on }[0, T], \forall T \in \mathbb{R}\right\} .
$$

If $\sigma$ is exact then we can pick a primitive $\theta$ of $\sigma$ and consider the same definition on $T M$. In this case we have:

$c:=\inf \left\{k \in \mathbb{R}: \mathbb{A}_{L+\theta+k}(\gamma) \geq 0 \forall\right.$ a.c. closed homotopically trivial curves defined on $\left.[0, T], \forall T \in \mathbb{R}\right\} ;$

$c_{0}:=\inf \left\{k \in \mathbb{R}: \mathbb{A}_{L+\theta+k}(\gamma) \geq 0 \forall\right.$ a.c. closed homologically trivial curves defined on $\left.[0, T], \forall T \in \mathbb{R}\right\}$.

It is immediate from (2.6) that

$$
c(g, \sigma, U) \geq \max _{q \in M} U(q) .
$$

Let us also denote by

$$
e_{0}=e_{0}(g, \sigma, U):=\inf \left\{k \in \mathbb{R}: \pi\left(\Sigma_{k}\right)=M\right\} .
$$

For $k>e_{0}$ the intersection of $\Sigma_{k}$ with any fibre $T_{q}^{*} M$ is diffeomorphic to a sphere $S^{n-1}$. We always have $c \geq e_{0}$, and in a lot of cases the strict inequality $c>e_{0}$ holds (see [44, Theorem 1.3]). In all cases if $k>c$ then $k$ is necessarily a regular value of $H$.

Denote by $\mathcal{R}(M)$ the set of all (smooth) Riemannian metrics $g$ on $M$, and denote by $\Omega_{\mathrm{we}}^{2}(M)$ the set of closed weakly exact 2-forms on $M$.

\subsection{Definition. Denote by}

$$
\mathcal{O} \subseteq \mathcal{R}(M) \times \Omega_{\mathrm{we}}^{2}(M) \times C^{\infty}(M, \mathbb{R}) \times \mathbb{R}
$$

the set of quadruples $(g, \sigma, U, k)$ such that

$$
k>c(g, \sigma, U) .
$$

It will be important later on to know how the critical value scales when we scale $\sigma$. Specifically, let us note the following lemma, whose proof is immediate from (2.5) and (2.6). 
2.2. Lemma. Given $s \in[0,1]$ it holds that

$$
\begin{gathered}
c\left(g, s \sigma, s^{2} U\right)=s^{2} c(g, \sigma, U) ; \\
c_{0}\left(g, s \sigma, s^{2} U\right)=s^{2} c(g, \sigma, U) .
\end{gathered}
$$

\subsection{Symplectic atoroidality.}

We remind the reader that $\sigma \in \Omega^{2}(M)$ is a weakly exact 2 -form whose pullback $\widetilde{\sigma} \in \Omega^{2}(\widetilde{M})$ admits a bounded primitive $\theta$. In this subsection we state and prove the key observation mentioned in the introduction that implies that the symplectic form $\omega$ is symplectically atoroidal. A similar idea appeared in Niche [42], although there the additional assumption was made that $M$ admits a metric of negative curvature. Here we require only the weaker assumption that $\widetilde{\sigma}$ is weakly exact and admits a bounded primitive].

The key lemma we use is the following, which originally appeared in [38, Lemma 2.2]. In the statement, $\mathbb{T}^{2}$ denotes the 2 -torus.

2.3. LEMMA. For any smooth map $f: \mathbb{T}^{2} \rightarrow M, f^{*} \sigma$ is exact.

Proof. Consider $G:=f_{*}\left(\pi_{1}\left(\mathbb{T}^{2}\right)\right) \leq \pi_{1}(M)$. Then $G$ is amenable, since $\pi_{1}\left(\mathbb{T}^{2}\right)=\mathbb{Z}^{2}$, which is amenable. Then [43, Lemma 5.3] tells us that since $\|\theta\|_{\infty}<\infty$ we can replace $\theta$ by a $G$-invariant primitive $\theta^{\prime}$ of $\widetilde{\sigma}$, which descends to define a primitive $\theta^{\prime \prime} \in \Omega^{1}\left(\mathbb{T}^{2}\right)$ of $f^{*} \sigma$.

Given a free homotopy class $\alpha \in\left[S^{1}, M\right]$, fix a reference loop $x_{\alpha}=\left(q_{\alpha}, p_{\alpha}\right) \in \Lambda_{\alpha} T^{*} M$. It will be convenient to insist that $x_{-\alpha}(t)=x_{\alpha}(-t)$, and that $x_{0}$ has image in one fibre, that is, $q_{0}$ is constant. Let $C:=S^{1} \times[0,1]$. Let $\bar{x} \in C^{0}\left(C, T^{*} M\right) \cap W^{1,2}\left(C, T^{*} M\right)$ denote any map such that $\bar{x}(\cdot, 0)=x$ and $\bar{x}(\cdot, 1)=x_{\alpha}$. Then thanks to the previous lemma the integral $\int_{C} \bar{x}^{*} \pi^{*} \sigma$ is is independent of the choice of $\bar{x}$. Similarly given any $q \in \Lambda_{\alpha} M$, let $\bar{q} \in C^{0}(C, M) \cap W^{1,2}(C, M)$ denote any map such that $\bar{q}(\cdot, 0)=q$ and $\bar{q}(\cdot, 1)=q_{\alpha}$. Then the integral $\int_{C} \bar{q}^{*} \sigma$ is independent of the choice of $\bar{q}$. Note that in particular if $q=\pi \circ x$ then

$$
\int_{C} \bar{x}^{*} \pi^{*} \sigma=\int_{C} \bar{q}^{*} \sigma
$$

and hence, recalling that $\lambda_{0}=p d q$ is the Liouville 1-form on $T^{*} M$, it holds that

$$
\int_{C} \bar{x}^{*} \omega=\int_{S^{1}} x^{*} \lambda_{0}+\int_{C} \bar{x}^{*} \pi^{*} \sigma=\int_{S^{1}} x^{*} \lambda_{0}+\int_{C} \bar{q}^{*} \sigma .
$$

\section{THE FREE TIME ACTION FUNCTIONAL}

\subsection{The definition of $S_{L+k}$.}

The first functional we work with is defined on $\Lambda M \times \mathbb{R}^{+}$. Given a potential $U \in C^{\infty}(M, \mathbb{R})$ and $k \in \mathbb{R}$ let

$$
L(q, v):=\frac{1}{2}|v|^{2}-U(q)
$$

and define the free time action functional $S_{L+k}: \Lambda M \times \mathbb{R}^{+} \rightarrow \mathbb{R}$ by

$$
S_{L+k}(q, T):=T \int_{S^{1}}\left(L\left(q(t), \frac{\dot{q}(t)}{T}\right)+k\right) d t+\int_{C} \bar{q}^{*} \sigma .
$$

This is well defined by the observations in the previous section. Moreover $S_{L+k} \in C^{2}\left(\Lambda M \times \mathbb{R}^{+}, \mathbb{R}\right)$; see [38, p195]. Let Crit $\left(S_{L+k}\right)$ denote the set of critical points of $S_{L+k}$, and given $\alpha \in\left[S^{1}, M\right]$,

\footnotetext{
${ }^{4}$ This really is a weaker assumption; if $M$ admits a metric of negative curvature then any closed 2-form in $M$ has bounded primitives in $\widetilde{M}$ [30], whilst the converse is clearly not true.
} 
let $\operatorname{Crit}\left(S_{L+k} ; \alpha\right)$ denote $\operatorname{Crit}\left(S_{L+k}\right) \cap\left(\Lambda_{\alpha} M \times \mathbb{R}^{+}\right)$. Given an interval $(a, b) \subseteq \mathbb{R}$, denote by Crit $^{(a, b)}\left(S_{L+k}\right)$ the set $\operatorname{Crit}\left(S_{L+k}\right) \cap S_{L+k}^{-1}((a, b))$. This functional was introduced in [38], and is a way of defining the free time action functional previously studied in [24, 22] when the magnetic form $\sigma$ is not exact.

It will be convenient to study what is essentially the lift of $S_{L+k}$ to the universal cover $\widetilde{M}$. Let $\widetilde{U}$ denote a lift of $U$ to $\widetilde{M}$. Let $\widetilde{E}: T \widetilde{M} \rightarrow \mathbb{R}$ denote the energy of the Lagrangian $\widetilde{L}$ :

$$
\widetilde{E}(q, v):=\frac{\partial \widetilde{L}}{\partial v}(q, v)(v)-\widetilde{L}(q, v) .
$$

Fix a primitive $\theta$ of the lifted form $\widetilde{\sigma}$ on $\widetilde{M}$ with $\|\theta\|_{\infty}<\infty$, and consider again the Lagrangian $\widetilde{L}+\theta: T \widetilde{M} \rightarrow \mathbb{R}$. Define

$$
\mathbb{S}_{\widetilde{L}+\theta+k}: W^{1,2}([0,1], \widetilde{M}) \times \mathbb{R}^{+} \rightarrow \mathbb{R}
$$

by

$$
\mathbb{S}_{\widetilde{L}+\theta+k}(q, T):=T \int_{0}^{1}\left(\widetilde{L}\left(q(t), \frac{\dot{q}(t)}{T}\right)+\frac{1}{T} \theta_{q(t)}(\dot{q}(t))+k\right) d t .
$$

In other words, $\mathbb{S}_{\widetilde{L}+\theta+k}$ is the standard free time action functional of the Lagrangian $\widetilde{L}+\theta$ and the energy level $k$. The free time action functional has been studied extensively in [24, 22]. We wish to relate the functional $\mathbb{S}_{\widetilde{L}+\theta+k}$ to that of $S_{L+k}$. For each $\alpha \in\left[S^{1}, M\right]$, fix a lift $\widetilde{q}_{\alpha}:[0,1] \rightarrow \widetilde{M}$ of our reference loops $q_{\alpha} \in \Lambda_{\alpha} M$. Define

$$
I(\alpha, \theta):=\int_{0}^{1}\left(\widetilde{q}_{\alpha}^{-}\right)^{*} \theta
$$

(where $\widetilde{q}_{\alpha}^{-}(t):=\widetilde{q}_{\alpha}(-t)$ ). Note that as $q_{0}$ is constant, $I(0, \theta)=0$. It is shown in [38, p8] that given $q \in \Lambda_{\alpha} M$ and $\widetilde{q}$ a lift of $q$ and $\bar{q}: C \rightarrow M$ a map as above that

$$
\int_{C} \bar{q}^{*} \sigma=\int_{0}^{1} \widetilde{q}^{*} \theta+I(\alpha, \theta)
$$

from which it follows that

$$
S_{L+k}(q, T)=\mathbb{S}_{\widetilde{L}+\theta+k}(\widetilde{q}, T)+I(\alpha, \theta) .
$$

Since $\|\theta\|_{\infty}<\infty$, we can find constants $e_{1}, e_{2}, f_{1}, f_{2}, g_{1}, g_{2}>0$ such that for all $(q, v) \in T \widetilde{M}$ it holds that

$$
\begin{gathered}
f_{1}|v|^{2}+f_{2} \geq(\widetilde{L}+\theta)(q, v) \geq e_{1}|v|^{2}-e_{2} ; \\
\widetilde{E}(q, v) \geq g_{1}|v|^{2}-g_{2} .
\end{gathered}
$$

Given any $(q, T) \in \Lambda_{\alpha} M \times \mathbb{R}^{+}$, let $\widetilde{q}:[0,1] \rightarrow \widetilde{M}$ denote a lift of $q$ and define $\gamma:[0, T] \rightarrow \widetilde{M}$ by $\gamma(t):=\widetilde{q}(t / T)$. One computes

$$
\begin{aligned}
\frac{\partial S_{L+k}}{\partial T}(q, T) & =\frac{1}{T} \int_{0}^{T}(k-\widetilde{E}(\gamma, \dot{\gamma})) d t \\
& \left.\leq \frac{1}{T} \int_{0}^{T}\left(k-\frac{g_{1}}{f_{1}} \widetilde{(L}+\theta\right)(\gamma, \dot{\gamma})+\frac{g_{1} f_{2}}{f_{1}}+g_{2}\right) d t \\
& =\frac{g_{1} f_{2}}{f_{1}}+g_{2}+\left(1+\frac{g_{1}}{f_{1}}\right) k-\frac{g_{1}}{f_{1} T} \mathbb{S}_{\widetilde{L}+\theta+k}(\widetilde{q}, T) \\
& =\frac{g_{1} f_{2}}{f_{1}}+g_{2}+\left(1+\frac{g_{1}}{f_{1}}\right) k-\frac{g_{1}}{f_{1} T} S_{L+k}(q, T)+\frac{g_{1} I(\alpha, \theta)}{f_{1} T} .
\end{aligned}
$$


In particular, in the case $\alpha=0$, since $I(0, \theta)=0$ we have proved the following lemma.

3.1. LEMMA. There exists $h_{0}>0$ such that if $(q, T) \in \Lambda_{0} M \times \mathbb{R}^{+}$and

$$
S_{L+k}(q, T)>h_{0} T
$$

then

$$
\frac{\partial S_{L+k}}{\partial T}(q, T)<0
$$

Let us recall a few definitions. If $S: \mathcal{M} \rightarrow \mathbb{R}$ is a $C^{2}$ functional on a Hilbert manifold $\mathcal{M}$ equipped with a Riemannian metric $G$, we say that $S$ satisfies (PS) $)_{a}$, that is, Palais-Smale condition at the level $a \in \mathbb{R}$, if any sequence $\left(x_{i}\right) \subseteq \mathcal{M}$ such that $S\left(x_{i}\right) \rightarrow a$ and $\left\|\nabla S\left(x_{i}\right)\right\| \rightarrow 0$ admits a convergent subsequence (where the gradient $\nabla S$ is taken with respect to $G$ ). Let $\Psi_{\tau}$ denote the local flow defined by the vector field $-\nabla S$, and let $\left(\tau_{-}(x), \tau_{+}(x)\right) \subseteq \overline{\mathbb{R}}$ denote the maximal interval of existence of the flow line $\tau \mapsto \Psi_{\tau}(x)$.

The next result is the key to defining the Morse (co)complex of $S_{L+k}$ (compare [4, Proposition 11.1, Proposition 11.2]). Recall the definition of the set $\mathcal{O}$ from Definition 2.1.

\subsection{THEOREM. (Properties of $S_{L+k}$ for $k>c(g, \sigma, U)$ )}

Fix $(g, \sigma, U, k) \in \mathcal{O}$. Let $\Psi_{\tau}$ denote the local flow of $-\nabla S_{L+k}$, where the gradient is taken with respect to the metric $\langle\langle\cdot, \cdot\rangle\rangle_{g}$ from (2.1). Then:

(1) $S_{L+k}$ is bounded below on $\Lambda M \times \mathbb{R}^{+}$and strictly positive on $\Lambda_{0} M \times \mathbb{R}^{+}$. Moreover

$$
\inf _{\Lambda_{0} M \times \mathbb{R}^{+}} S_{L+k}=0, \inf _{\operatorname{Crit}\left(S_{L+k} ; 0\right)} S_{L+k}>0 .
$$

(2) If $\alpha \in\left[S^{1}, M\right]$ is a non-trivial free homotopy class then $\tau_{+}(q, T)=\infty$ for all $(q, T) \in$ $\Lambda_{\alpha} M \times \mathbb{R}^{+}$. If $(q, T) \in \Lambda_{0} M \times \mathbb{R}^{+}$and $\tau_{+}(q, T)<\infty$ then if $\left(q_{\tau}, T_{\tau}\right):=\Psi_{\tau}(q, T)$ then $S_{L+k}\left(q_{\tau}, T_{\tau}\right) \rightarrow 0, T_{\tau} \rightarrow 0$ and $q_{\tau}$ converges to a constant loop as $\tau \uparrow \tau_{+}(q, T)$. In particular this happens if

$$
S_{L+k}(q, T)<\inf _{\operatorname{Crit}\left(S_{L+k} ; 0\right)} S_{L+k} .
$$

(3) If $\alpha \in\left[S^{1}, M\right]$ is a non-trivial free homotopy class then $\tau_{-}(q, T)=-\infty$ for all $(q, T) \in$ $\Lambda_{\alpha} M \times \mathbb{R}^{+}$.

(4) There exists $h_{1}>0$ with the following properties: given $S>0$ define

$$
\mathcal{A}(S):=\left\{\left.S_{L+k}\right|_{\Lambda_{0} M \times \mathbb{R}^{+}}<S\right\} \cap\left\{T<h_{1} S\right\}
$$

Then $\mathcal{A}(S) \cap \operatorname{Crit}\left(S_{L+k}\right)=\emptyset$ for all $S>0$, and for any $S>0$, if $(q, T) \in \mathcal{A}(S)$ then $\Psi_{\tau}(q, T) \in \mathcal{A}(S)$ for all $\tau \in\left(\tau_{-}(q, T), 0\right]$. Finally if $(q, T) \in \Lambda_{0} M \times \mathbb{R}^{+}$is such that $\tau_{-}(q, T)>-\infty$ and $S_{L+k}(q, T) \geq S$ then there exists $\tau<0$ such that $\Psi_{\tau}(q, T) \in \mathcal{A}(S)$.

Proof. The fact that $S_{L+k}$ is bounded below is proved in [38, Lemma 4.2]. The fact that $S_{L+k}$ is strictly positive on $\Lambda_{0} M \times \mathbb{R}^{+}$follows from the fact that given $(q, T) \in \Lambda_{0} M \times \mathbb{R}^{+}$we have

$$
S_{L+k}(q, T)=\mathbb{S}_{\widetilde{L}+\theta+k}(\widetilde{q}, T)=\mathbb{S}_{\widetilde{L}+\theta+c}(\widetilde{q}, T)+(k-c) T \geq 0+(k-c) T .
$$

If $q$ is a constant loop then $\lim _{T \rightarrow 0} S_{L+k}(q, T)=0$, and hence the infimum of $S_{L+k}$ on $\Lambda_{0} M \times \mathbb{R}^{+}$is zero. To see that the infimum of $S_{L+k}$ on $\operatorname{Crit}\left(S_{L+k} ; 0\right)$ is strictly positive, we use Lemma 4.1 , to be proved in the next section, which says that $(q, T) \in \operatorname{Crit}\left(S_{L+k}\right)$ if and only if $(x, T) \in \operatorname{Crit}\left(A_{H-k}\right)$, where $x=(q, \dot{q}) \in \Lambda T^{*} M$. Since $\Sigma_{k}:=H^{-1}(k)$ is compact and $k$ is a regular value of $H$, the period of its Hamiltonian orbits is bounded away from zero, and thus

$$
\inf \left\{\eta>0:(x, \eta) \in \operatorname{Crit}\left(A_{H-k}\right)\right\}>0 .
$$

\footnotetext{
${ }^{5}$ Strictly speaking, all the proofs in [38] are given only in the special case $U \equiv 0$, but there are no changes in this case.
} 
Thus the infimum of $S_{L+k}$ on $\operatorname{Crit}\left(S_{L+k} ; 0\right)$ is strictly positive. This proves (1).

Statement (2) is proved in [38, Theorem 3.2, Lemma 4.4]. Since $S_{L+k}$ is bounded below, if $(q, T) \in$ $\Lambda M \times \mathbb{R}^{+}$is such that $\tau_{+}(q, T)<\infty$ then if $\left(q_{\tau}, T_{\tau}\right):=\Psi_{\tau}(q, T)$, we must have $\lim _{\tau \uparrow \tau_{+}(q, T)} T_{\tau}=0$ (see for instance [37, Proposition 8.4]). This can only happen if $(q, T) \in \Lambda_{0} M \times \mathbb{R}^{+}$, since if $q$ is non-contractible then $T$ is bounded away from zero ([38, Lemma 4.3]). If $(q, T) \in \Lambda_{0} M \times \mathbb{R}^{+}$then we have

$$
\begin{aligned}
\frac{\partial T_{\tau}}{\partial \tau} & =\left\langle\left\langle\frac{\partial}{\partial \tau} \Psi_{\tau}(q, T),\left(0, \frac{\partial}{\partial T}\right)\right\rangle\right\rangle_{g} \\
& =-\frac{\partial S_{L+k}}{\partial T}\left(q_{\tau}, T_{\tau}\right),
\end{aligned}
$$

and thus Lemma 3.1 tells us that if $S_{L+k}\left(q_{\tau}, T_{\tau}\right)>h_{0} T_{\tau}$ then $\frac{\partial T_{\tau}}{\partial \tau}>0$. Thus the decreasing function $\tau \mapsto S_{L+k}\left(q_{\tau}, T_{\tau}\right)$ must converge to zero. Using (3.4) it is easy to see that the fact that both $S_{L+k}\left(q_{\tau}, T_{\tau}\right)$ and $T_{\tau}$ tend to zero implies that $\int_{S^{1}}\left|\dot{q}_{\tau}(t)\right|^{2} d t$ also tends to zero as $\tau \uparrow \tau_{+}(q, T)$. This proves (3). The proof of (4) follows in exactly the same way (see [4, Proposition 11.2]).

\subsection{Fixing the period.}

It will be useful to consider the fixed period action functional. Given $T \in \mathbb{R}^{+}$let us denote by $S_{L+k}^{T}: \Lambda M \rightarrow \mathbb{R}$ the functional defined by

$$
S_{L+k}^{T}(q):=S_{L+k}(q, T) .
$$

Note that

$$
d_{q} S_{L+k}^{T}(\zeta)=d_{(q, T)} S_{L+k}(\zeta, 0)
$$

Thus if $(q, T) \in \operatorname{Crit}\left(S_{L+k}\right)$ then $q \in \operatorname{Crit}\left(S_{L+k}^{T}\right)$.

\subsection{The Morse index and the non-degeneracy assumption.}

By definition, the Morse index $i(q, T)$ of a critical point $(q, T) \in \operatorname{Crit}\left(S_{L+k}\right)$ is the maximal dimension of a subspace $W \subseteq W^{1,2}\left(S^{1}, q^{*} T M\right) \times \mathbb{R}$ on which the Hessian $\nabla_{(q, T)}^{2} S_{L+k}$ of of $S_{L+k}$ at $(q, T)$ is negative definite. It is well known that for the Lagrangians $L=L_{g}-\pi^{*} U$ that we work with the Morse index $i(q, T)$ is always finite [25, Section 1]. Similarly let $i_{T}(q)$ denote the Morse index of a critical point $q \in \operatorname{Crit}\left(S_{L+k}^{T}\right)$, that is, the dimension of a maximal subspace of $W^{1,2}\left(S^{1}, q^{*} T M\right)$ on which the Hessian $\nabla_{q}^{2} S_{L+k}^{T}$ of the $S_{L+k}$ at $(q, T)$ (this time taken with respect to the $W^{1,2}$ metric on $\Lambda M)$ is negative definite.

3.3. Definition. Let us say that a critical point $(q, T) \in \operatorname{Crit}\left(S_{L+k}\right)$ is non-degenerate if the kernel of $\nabla_{q}^{2} S_{L+k}^{T}$ is one-dimensional, spanned by the vector $\dot{q} \in T_{q} \Lambda M$.

Suppose $(q, T)$ is a non-degenerate critical point. One consequence of this assumption (cf. the discussion at the start of Section 4.3) is the existence of an orbit cylinder about $(q, T)$. That is, there exists $\varepsilon>0$ and a unique smooth (in $s$ ) family $\left(q_{s}, T_{s}\right) \in \operatorname{Crit}\left(S_{L+k+s}\right)$ for $s \in(-\varepsilon, \varepsilon)$, where $\left(q_{0}, T_{0}\right)=(q, T)$. Moreover $\frac{\partial T_{s}}{\partial s}(0) \neq 0$. Given such a non-degenerate critical point $(q, T)$, we may therefore define

$$
\chi(q, T):=\operatorname{sign}\left(-\frac{\partial T_{s}}{\partial s}(0)\right) \in\{-1,1\} .
$$

Recall that a function $S: \mathcal{M} \rightarrow \mathbb{R}$ on on a Hilbert manifold $\mathcal{M}$ equipped with a Riemannian metric $G$ is called Morse-Bott if the set $\operatorname{Crit}(S)$ of its critical points is a submanifold of $\mathcal{M}$ (possibly with components of differing dimensions) and such that for each $x \in \operatorname{Crit}(S)$, the Hessian $\nabla_{x}^{2} S$ of $S$ (defined with respect to $G$ ) is a Fredholm operator and satisfies

$$
\text { ker } \nabla_{x}^{2} S=T_{x} \operatorname{Crit}(S) \text {. }
$$


Denote by

$$
\mathcal{O}_{\text {reg }} \subseteq \mathcal{O}
$$

the set of quadruples $(g, \sigma, U, k) \in \mathcal{O}$ with the property that if $L:=L_{g}-\pi^{*} U$ then every critical point of $S_{L+k}$ is non-degenerate. In this case $S_{L+k}$ is a Morse-Bott function, and Crit $\left(S_{L+k}\right)$ consists of a discrete union of circles. The following theorem can be proved by adapting the proofs of [16, Theorem B1] (see also the Corrigendum [18]) together with a version of the Klingenberg-Takens theorem [33] for magnetic flows. Full details can be found in [40].

3.4. Theorem. Suppose $(g, \sigma, U, k) \in \mathcal{O}$. Then given any $\varepsilon>0$ there exists $g^{\prime} \in \mathcal{R}(M)$ with $\left\|g-g^{\prime}\right\|_{\infty}<\varepsilon$ such that $\left(g^{\prime}, \sigma, U, k\right) \in \mathcal{O}_{\text {reg. }}$

The following theorem is proved in [41].

3.5. Theorem. Assume $(g, \sigma, U, k) \in \mathcal{O}_{\text {reg }}$, and set $L:=L_{g}-\pi^{*} U$. Let $(q, T) \in \operatorname{Crit}\left(S_{L+k}\right)$. Then

$$
i(q, T)=i_{T}(q)+\frac{1}{2}-\frac{1}{2} \chi(q, T) .
$$

3.6. REMARK. In [4, Section 10] Abbondandolo and Schwarz work with a Lagrangian which is the Fenchel transform of a Hamiltonian which is homogeneous of degree 2 in a neighborhood of $\Sigma_{k}$. In this case one can show $\chi(q, T)=+1$ for every critical point $(q, T)$, and hence the Morse index of the free time action functional always agrees with the corresponding index of the fixed period action functional. In the more general situation that we are interested in here however it is possible that there exist critical points $(q, T)$ with $\chi(q, T)=-1$. In [41] we provide an example of an exact magnetic Lagrangian $L: T S^{2} \rightarrow \mathbb{R}$ for which there exists a non-degenerate critical point $(q, T)$ of $S_{L+k}$ for $k>c$ such that $\chi(q, T)=-1$.

\subsection{The Morse (co)chain complex.}

In this section we construct the Morse co(chain) complex and state the Morse homology theorem, which says that the corresponding Morse (co)homology coincides with the singular (co)homology of the free loop space $\Lambda M$. Fix $(g, \sigma, U, k) \in \mathcal{O}_{\text {reg }}$, and put $L:=L_{g}-\pi^{*} U$.

It will be convenient to put

$$
\overline{\operatorname{Crit}}\left(S_{L+k}\right):=\operatorname{Crit}\left(S_{L+k}\right) \cup(M \times\{0\}),
$$

where points in $M$ should be thought of as constant loops in $\Lambda M$. We refer to elements of the set $\overline{\operatorname{Crit}}\left(S_{L+k}\right) \backslash \operatorname{Crit}\left(S_{L+k}\right)$ as critical points at infinity 6 .

We will need three pieces of auxiliary data to define the Morse (co)complex. Firstly, let $G$ denote a metric on $\Lambda M \times \mathbb{R}^{+}$that is a generic perturbation of the metric $\langle\langle\cdot, \cdot\rangle\rangle_{g}$ (in particular $G$ should be uniformly equivalent to $\langle\langle\cdot, \cdot\rangle\rangle_{g}$ ). Write $\Psi_{\tau}$ for the flow of $-\nabla S_{L+k}$, now taken with respect to the metric $G$. Secondly, let $f: \overline{\operatorname{Crit}}\left(S_{L+k}\right) \rightarrow \mathbb{R}$ denote a Morse function on $\overline{\operatorname{Crit}}\left(S_{L+k}\right)$, and write $\overline{\operatorname{Crit}}(f) \subseteq \overline{\operatorname{Crit}}\left(S_{L+k}\right)$ for the set of critical points of $f$, and $\operatorname{Crit}(f):=\operatorname{Crit}\left(S_{L+k}\right) \cap \overline{\operatorname{Crit}}(f)$. Thirdly, let $g_{0}$ denote a Riemannian metric on $\overline{\operatorname{Crit}}\left(S_{L+k}\right)$ such that the flow $\phi_{t}^{-\nabla f}$ of $-\nabla f$ is Morse-Smale. The Morse-Smale assumption implies that for every pair $w_{-}, w_{+}$of critical points of $f$ the unstable manifold $W^{u}\left(w_{-} ;-\nabla f\right)$ intersects the stable manifold $W^{s}\left(w_{+} ;-\nabla f\right)$ transversely. Denote by $i_{f}(z):=\operatorname{dim} W^{u}(w ;-\nabla f)$ the Morse index of a critical point $z \in \overline{\operatorname{Crit}}(f)$.

Finally for $w \in \overline{\operatorname{Crit}}(f)$ write

$$
\widehat{i}_{f}(w):=i(w)+i_{f}(w)
$$

where by definition we put $i(w)=0$ for $w \in \overline{\operatorname{Crit}}\left(S_{L+k}\right) \backslash \operatorname{Crit}\left(S_{L+k}\right)$. Let

$$
\overline{\operatorname{Crit}}_{i}(f):=\left\{w \in \overline{\operatorname{Crit}}(f): \widehat{i}_{f}(w)=i\right\} .
$$

\footnotetext{
${ }^{6}$ In a lot of ways this is a poor choice of name, as these critical points lie at $T=0$, not at $T=\infty$ !
} 
Given $w_{-}, w_{+} \in \overline{\operatorname{Crit}}(f)$, denote by

$$
\widetilde{\mathcal{W}}_{0}\left(w_{-}, w_{+}\right):=W^{u}\left(w_{-} ;-\nabla f\right) \cap W^{s}\left(w_{+} ;-\nabla f\right) .
$$

Let

$$
\mathcal{W}_{0}\left(w_{-}, w_{+}\right):=\widetilde{\mathcal{W}}_{0}\left(w_{-}, w_{+}\right) / \mathbb{R}
$$

denote the quotient of $\widetilde{\mathcal{W}}_{0}\left(w_{-}, w_{+}\right)$by the obvious free $\mathbb{R}$-action (if $w_{-}=w_{+}, \mathcal{W}_{0}\left(w_{-}, w_{+}\right)=\emptyset$ ).

Suppose now that $w_{-} \in \operatorname{Crit}(f)$, that is, $w_{-}$is not a critical point at infinity. If $m \in \mathbb{N}$ and $w_{+} \in \overline{\operatorname{Crit}}(f)$, let $\widetilde{\mathcal{W}}_{m}\left(w_{-}, w_{+}\right)$denote the set of tuples $\boldsymbol{w}=\left(w_{1}, \ldots, w_{m}\right)$ where each $w_{i} \in(\Lambda M \times$ $\left.\mathbb{R}^{+}\right) \backslash \operatorname{Crit}\left(S_{L+k}\right)$ is such that

$$
\Psi_{-\infty}\left(w_{1}\right) \in W^{u}\left(w_{-} ;-\nabla f\right), \ldots, \Psi_{\infty}\left(w_{m}\right) \in W^{s}\left(w_{+} ;-\nabla f\right),
$$

and such that

$$
\Psi_{-\infty}\left(w_{i+1}\right) \in \phi_{\mathbb{R}^{+}}^{-\nabla f}\left(\Psi_{\infty}\left(w_{i}\right)\right) .
$$

Note that if $m \geq 1$ then $\widetilde{\mathcal{W}}_{m}\left(w^{-}, w^{+}\right)$admits a free action of $\mathbb{R}^{m}$ via

$$
\left(w_{1}, \ldots, w_{m}\right) \mapsto\left(\Psi_{s_{1}}\left(w_{1}\right), \ldots, \Psi_{s_{m}}\left(w_{m}\right)\right), \quad\left(s_{1}, \ldots, s_{m}\right) \in \mathbb{R}^{m} .
$$

We denote by $\mathcal{W}_{m}\left(w_{-}, w_{+}\right)$the quotient of $\widetilde{\mathcal{W}}_{m}\left(w_{-}, w_{+}\right)$by this action. Put

$$
\mathcal{W}\left(w_{-}, w_{+}\right):=\bigcup_{m \in \mathbb{N} \cup\{0\}} \mathcal{W}_{m}\left(w_{-}, w_{+}\right) .
$$

Finally if $w_{-} \in \overline{\operatorname{Crit}}(f) \backslash \operatorname{Crit}(f)$ is a critical point at infinity, set

$$
\widetilde{\mathcal{W}}_{m}\left(w_{-}, w_{+}\right)=\mathcal{W}_{m}\left(w_{-}, w_{+}\right):=\emptyset
$$

for all $m \in \mathbb{N}$ and $w_{+} \in \overline{\operatorname{Crit}}(f)$, so that $\mathcal{W}\left(w_{-}, w_{+}\right)=\mathcal{W}_{0}\left(w_{-}, w_{+}\right)$.

The next theorem, together with Theorem 3.8 below, follows from Theorem 3.2 exactly as in [4, Section 11]. See also [28, Appendix A] for more information.

3.7. THEOREM. For a generic choice of $G$ and $g_{0}$ the $\operatorname{set} \mathcal{W}\left(w_{-}, w_{+}\right)$is a finite dimensional smooth manifold of dimension

$$
\operatorname{dim} \mathcal{W}\left(w_{-}, w_{+}\right)=\widehat{i}_{f}\left(w_{-}\right)-\widehat{i}_{f}\left(w_{+}\right)-1
$$

Moreover if $\widehat{i}_{f}\left(w_{-}\right)-\widehat{i}_{f}\left(w_{+}\right)=1$ then $\mathcal{W}\left(w_{-}, w_{+}\right)$is compact, and hence a finite set.

If $\widehat{i}_{f}\left(w_{-}\right)-\widehat{i}_{f}\left(w_{+}\right)=1$ we may therefore define

$$
n_{\text {Morse }}\left(w_{-}, w_{+}\right):=\# \mathcal{W}\left(w_{-}, w_{+}\right), \quad \text { taken } \bmod 2 .
$$

Put

$$
C M_{i}\left(S_{L+k}, f\right):=\bigoplus_{w \in \overline{\operatorname{Crit}}_{i}(f)} \mathbb{Z}_{2} w, \quad C M^{i}\left(S_{L+k}, f\right):=\prod_{w \in \overline{\operatorname{Crit}}_{i}(f)} \mathbb{Z}_{2} w .
$$

Define

$$
\partial^{\text {Morse }}=\partial^{\text {Morse }}\left(G, g_{0}\right): C M_{i}\left(S_{L+k}, f\right) \rightarrow C M_{i-1}\left(S_{L+k}, f\right)
$$

by

$$
\partial^{\text {Morse }} w=\sum_{w^{\prime} \in \overline{\operatorname{Crit}}_{i-1}(f)} n_{\text {Morse }}\left(w, w^{\prime}\right) w^{\prime}
$$

Define

$$
\delta^{\text {Morse }}=\delta^{\text {Morse }}\left(G, g_{0}\right): C M^{i}\left(S_{L+k}, f\right) \rightarrow C M^{i+1}\left(S_{L+k}, f\right)
$$


by

$$
\delta^{\text {Morse }} w:=\sum_{w^{\prime} \in \operatorname{Crit}_{i+1}(f)} n_{\text {Morse }}\left(w^{\prime}, w\right) w^{\prime} .
$$

The next result is the Morse homology theorem.

3.8. Theorem. Let $G$ and $g_{0}$ be as Theorem 3.7 Then it holds that $\partial^{\text {Morse }} \circ \partial^{\text {Morse }}=0$ and also that $\delta^{\text {Morse }} \circ \delta^{\text {Morse }}=0$. Thus $\left\{C M_{*}\left(S_{L+k}, f\right), \partial^{\text {Morse }}\left(G, g_{0}\right)\right\}$ and $\left\{C M^{*}\left(S_{L+k}, f\right), \delta^{\text {Morse }}\left(G, g_{0}\right)\right\}$ form a chain (respectively cochain) complex. The isomorphism class of these complexes is independent of the choice of $f$, $G$ and $g_{0}$. The associated (co)homology, known as the Morse (co)homology of $S_{L+k}$ is isomorphic to the singular (co)homology of $\Lambda M \times \mathbb{R}^{+}$:

$$
H M_{*}\left(S_{L+k}\right) \cong H_{*}\left(\Lambda M \times \mathbb{R}^{+} ; \mathbb{Z}_{2}\right), \quad H M^{*}\left(S_{L+k}\right) \cong H^{*}\left(\Lambda M \times \mathbb{R}^{+} ; \mathbb{Z}_{2}\right)
$$

Moreover this isomorphism respects the splitting $\Lambda M=\bigoplus_{\alpha \in\left[S^{1}, M\right]} \Lambda_{\alpha} M$ : if $C M_{*}\left(S_{L+k}, f ; \alpha\right)$ denotes the subcomplex of $C M_{*}\left(S_{L+k}, f\right)$ generated by the critical points $w \in \overline{\operatorname{Crit}}(f) \cap \overline{\operatorname{Crit}}\left(S_{L+k} ; \alpha\right)$ then the homology $H M_{*}\left(S_{L+k} ; \alpha\right)$ of this subcomplex is isomorphic to $H_{*}\left(\Lambda_{\alpha} M \times \mathbb{R}^{+} ; \mathbb{Z}_{2}\right)$ under the isomorphism of the previous theorem. The same statements holds for cohomology: $H M^{*}\left(S_{L+k} ; \alpha\right) \cong$ $H^{*}\left(\Lambda_{\alpha} M \times \mathbb{R}^{+}\right)$.

\section{THE RABINOWITZ ACTION FUNCTIONAL}

In this section we finally define the Rabinowitz action functional, and its associated Rabinowitz Floer homology.

\subsection{Definition of the Rabinowitz action functional.}

Fix an autonomous potential $U \in C^{\infty}(M, \mathbb{R})$, and put $H=H_{g}+\pi^{*} U$. Fix a regular value $k \in \mathbb{R}$ of $H$, and put $\Sigma_{k}:=H^{-1}(k)$. We define the Rabinowitz action functional $A_{H-k}: \Lambda T^{*} M \times \mathbb{R} \rightarrow \mathbb{R}$ by

$$
\begin{aligned}
A_{H-k}(x, \eta): & =\int_{C} \bar{x}^{*} \omega-\eta \int_{S^{1}}(H(x(t))-k) d t \\
& =\int_{S^{1}} x^{*} \lambda_{0}+\int_{C} \bar{x}^{*} \pi^{*} \sigma-\eta \int_{S^{1}}(H(x(t))-k) d t
\end{aligned}
$$

(see Section 2.3 for the definition of the term $\int_{C} \bar{x}^{*} \omega$; the latter equality follows from (2.9)). Denote by $\operatorname{Crit}\left(A_{H-k}\right)$ the set of critical points of $A_{H-k}$, and given $\alpha \in\left[S^{1}, M\right]$, let $\operatorname{Crit}\left(A_{H-k} ; \alpha\right):=$ $\operatorname{Crit}\left(A_{H-k}\right) \cap\left(\Lambda_{\alpha} T^{*} M \times \mathbb{R}\right)$. Given an interval $(a, b) \subseteq \mathbb{R}$, denote by $\mathrm{Crit}^{(a, b)}\left(A_{H-k}\right)$ the set $\operatorname{Crit}\left(A_{H-k}\right) \cap A_{H-k}^{-1}((a, b))$.

The critical points of $A_{H-k}$ are easily seen to satisfy:

$$
\begin{gathered}
\dot{x}=\eta X_{H}(x(t)) \quad \text { for all } t \in S^{1} ; \\
\int_{S^{1}}(H(x(t))-k) d t=0 .
\end{gathered}
$$

Since $H$ is invariant under its Hamiltonian flow, the second equation implies

$$
H(x(t))-k=0 \text { for all } t \in S^{1},
$$

that is,

$$
x\left(S^{1}\right) \subseteq \Sigma_{k} .
$$

Thus we can characterize $\operatorname{Crit}\left(A_{H-k}\right)$ by

$$
\begin{gathered}
\operatorname{Crit}\left(A_{H-k}\right)=\left\{(x, \eta) \in \Lambda T^{*} M \times \mathbb{R}: x \in C^{\infty}\left(S^{1}, T^{*} M\right)\right. \\
\left.\dot{x}(t)=\eta X_{H}(x(t)), x\left(S^{1}\right) \subseteq \Sigma_{k}\right\} .
\end{gathered}
$$


The circle $S^{1}$ acts on $\Lambda T^{*} M$ via rotation:

$$
r_{*}(x)(t):=x(r+t), \quad r \in S^{1}, x \in \Lambda T^{*} M .
$$

This action extends to an action on $\Lambda T^{*} M \times \mathbb{R}$ by ignoring the $\mathbb{R}$-factor. Since $H$ is autonomous, the Rabinowitz action functional $A_{H-k}$ is invariant under this action. In particular, its critical set $\operatorname{Crit}\left(A_{H-k}\right)$ is invariant.

Thus the elements of $\operatorname{Crit}\left(A_{H-k}\right)$ come in two flavours. Firstly, for each periodic orbit $y: \mathbb{R} / T \mathbb{Z} \rightarrow$ $\Sigma_{k}$ of $X_{H}$ on $\Sigma_{k}$ with minimal period $T>0$, and for each $m \in \mathbb{Z} \backslash\{0\}$, we have a copy of $S^{1}$ :

$$
\left\{\left(r_{*}(y)(m T t), m T\right): r \in S^{1}\right\}
$$

contained in $\operatorname{Crit}\left(A_{H-k}\right)$. Secondly, $\operatorname{Crit}\left(A_{H-k}\right)$ contains the set $\left\{(x, 0): x \in \Sigma_{k}\right\}$, where a point in $\Sigma_{k}$ should be interpreted as a constant loop in $\Lambda T^{*} M$.

Let us fix a 1-periodic almost complex structure $J \in \mathcal{J}(\omega)$. We denote by $\nabla A_{H-k}$ the $L^{2}$-gradient of $A_{H-k}$ with respect to the $L^{2}$-metric $\langle\langle\cdot, \cdot\rangle\rangle_{J}$ :

$$
\nabla A_{H-k}(x, \eta)=\left(\begin{array}{c}
J(t, x)\left(\dot{x}-\eta X_{H}(x)\right. \\
-\int_{S^{1}}(H(x(t))-k) d t
\end{array}\right) .
$$

\subsection{Comparing the functionals $S_{L+k}$ and $A_{H-k}$.}

Let $H$ be as above and set $L:=L_{g}-\pi^{*} U$. The following lemma outlines the relationship between the critical points of $S_{L+k}$ and $A_{H-k}$. The proof is identical to the analogous statements in [4, Section 5], and will be omitted.

4.1. LEMMA. (Properties of $S_{L+k}$ and $A_{H-k}$ )

(1) Given $w=(q, T) \in \operatorname{Crit}\left(S_{L+k} ; \alpha\right)$, define

$$
Z^{+}(w):=(x, T) \in \Lambda_{\alpha} T^{*} M \times \mathbb{R}, \text { where } x(t):=(q(t), \dot{q}(t)),
$$

and define

$$
Z^{-}(w):=\left(x^{-},-T\right) \in \Lambda_{-\alpha} T^{*} M \times \mathbb{R}, \text { where } x^{-}(t):=x(-t) .
$$

Then $Z^{+}(w) \in \operatorname{Crit}\left(A_{H-k} ; \alpha\right)$ and $Z^{-}(w) \in \operatorname{Crit}\left(A_{H-k} ;-\alpha\right)$, and moreover the map

$$
\operatorname{Crit}\left(S_{L+k}\right) \times\{-1,1\} \rightarrow\left\{(x, \eta) \in \operatorname{Crit}\left(A_{H-k}\right): \eta \neq 0\right\}
$$

given by

is a bijection, and

$$
(w, \pm 1) \mapsto Z^{ \pm}(w)
$$

$$
A_{H-k}\left(Z^{ \pm}(w)\right)= \pm S_{L+k}(w) .
$$

(2) Given any $(x, \eta) \in \Lambda T^{*} M \times \mathbb{R}$ with $\eta>0$, if $q:=\pi \circ x$ then

$$
A_{H-k}(x, \eta) \leq S_{L+k}(q, \eta),
$$

with equality if and only if $x=(q, \dot{q})$. If $x^{-}(t):=x(-t)$ then

$$
A_{H-k}\left(x^{-},-\eta\right) \geq-S_{L+k}(q, \eta)
$$

with equality if and only if $x=(q, \dot{q})$.

(3) Let $w \in \operatorname{Crit}\left(S_{L+k}\right)$. Then for all $(\xi, b) \in T_{Z^{+}(w)}\left(\Lambda T^{*} M \times \mathbb{R}\right)$ it holds that

$$
d_{Z^{+}(w)}^{2} A_{H-k}((\xi, b),(\xi, b)) \leq d_{w}^{2} S_{L+k}((d \pi(\xi), b),(d \pi(\xi), b)),
$$

and similarly for all $(\xi, b) \in T_{Z^{-}(w)}\left(\Lambda T^{*} M \times \mathbb{R}\right)$ it holds that

$$
d_{Z^{-}(w)}^{2} A_{H-k}((\xi, b),(\xi, b)) \geq-d_{w}^{2} S_{L+k}\left(\left(d \pi(\xi)^{-},-b\right),\left(d \pi(\xi)^{-},-b\right)\right),
$$

where $d \pi(\xi)^{-}(t):=d \pi(\xi)(-t)$. 
(4) Given $w \in \operatorname{Crit}\left(S_{L+k}\right)$, a pair $(\xi, b)$ lies in the kernel of the Hessian of $A_{H-k}$ at $Z^{+}(w)$ if and only if the pair $(d \pi(\xi), b)$ lies in the kernel of the Hessian of $S_{L+k}$ at $w$, and similarly $(\xi, b)$ lies in the kernel of the Hessian of $A_{H-k}$ at $Z^{-}(w)$ if and only if the pair $\left(d \pi(\xi)^{-},-b\right)$ lies in the kernel of the Hessian of $S_{L+k}$ at $w$.

As an immediate corollary of the preceding lemma and the definition of $\mathcal{O}_{\text {reg }}$ (cf. Theorem 3.4) we obtain the following statement.

4.2. Corollary. If $(g, \sigma, U, k) \in \mathcal{O}_{\text {reg }}$ and $H:=H_{g}+\pi^{*} U$ then every periodic orbit of $H$ lying in $\Sigma_{k}$ is strongly transversely non-degenerate. In other words, if $y: \mathbb{R} / T \mathbb{Z} \rightarrow \Sigma_{k}$ is a periodic orbit of $X_{H}$ then the nullity of $y, \nu(y)$ satisfies

$$
\nu(y):=\operatorname{dim} \operatorname{ker}\left(d_{y(0)} \phi_{T}^{H}-\mathbb{1}\right)=1 .
$$

This implies that the Rabinowitz action functional $A_{H-k}$ is Morse-Bott, and $\operatorname{Crit}\left(A_{H-k}\right)$ consists of a copy of $\Sigma_{k} \times\{0\}$ together with a discrete union of circles.

Proof. It remains only to check that $A_{H-k}$ is Morse-Bott at the constant orbits $(x, 0) \in \Sigma_{k} \times\{0\} \subseteq$ $\operatorname{Crit}\left(A_{H-k}\right)$. A short computation tells us that $(\xi, b)$ lies in the kernel of the Hessian of $A_{H-k}$ at $(x, 0)$ if and only if

$$
\begin{gathered}
-\nabla_{t} \xi(t)+b X_{H}(x)=0 \\
\int_{S^{1}} d_{x} H(\xi(t)) d t=0 .
\end{gathered}
$$

Integrating the first equation and using the fact that $\xi$ is a loop and $X_{H}(x) \neq 0$ (as $k$ is a regular value of $H$ and $x \in \Sigma_{k}$ ), we see that $b=0$. Thus $\xi(t) \equiv \xi(0)$ is constant, and the second equation then says that $\xi(0) \in \operatorname{ker} d_{x} H=T_{x} \Sigma_{k}$. Thus $A_{H-k}$ is Morse-Bott at the constant orbits.

\subsection{Grading the Rabinowitz Floer complex.}

Fix $(g, \sigma, U, k) \in \mathcal{O}_{\text {reg. }}$. Let $H=H_{g}+\pi^{*} U$. Suppose $y: \mathbb{R} / T \mathbb{Z} \rightarrow \Sigma_{k}$ is a periodic orbit of $X_{H}$. Our non-degeneracy assumption on $y$ implies that there exists $\varepsilon>0$ together with a smooth (in $s$ ) family $y_{s}: \mathbb{R} / T_{s} \mathbb{Z} \rightarrow T^{*} M$ for $s \in(-\varepsilon, \varepsilon)$ of $T_{s}$-periodic orbits of $X_{H}$ with $y_{0}=y$ and $H\left(y_{s}\right) \equiv k+s$. Such a family $\left(y_{s}\right)$ is known as an orbit cylinder about $y$, and the family $\left(y_{s}\right)$ is unique. Actually the existence of such an orbit cylinder requires only that $y$ has exactly two Floquet multipliers equal to one (see for instance [31, Proposition 4.2]). Our non-degeneracy assumption is strictly stronger than this: it implies in addition that $\frac{\partial T_{s}}{\partial s}(0) \neq 0$. Indeed, let $N$ denote a hypersurface inside of $\Sigma_{k}$ which is transverse to $y(\mathbb{R} / T \mathbb{Z})$ at the point $y(0)$, with $T_{y(0)} N$ equal to the symplectic orthogonal to the tangent space of the orbit cylinder. Let $P_{y}: \mathcal{U} \rightarrow \mathcal{V}$ denote the associated Poincaré map, where $\mathcal{U}$ and $\mathcal{V}$ are neighborhoods of $y(0)$. $P$ is a diffeomorphism that fixes $y(0)$. Then there exists a unique symplectic splitting of $T_{y(0)} T^{*} M$ such that $d_{y(0)} \phi_{T}^{H}$ is given by

$$
d_{y(0)} \phi_{T}^{H}=\left(\begin{array}{ccc}
1 & -\frac{\partial T_{s}}{\partial s}(0) & 0 \\
0 & 1 & 0 \\
0 & 0 & d_{y(0)} P_{y}
\end{array}\right) .
$$

Here $\mathbb{1}-d_{y(0)} P_{z}$ is invertible. The assumption that $\nu(y)=1$ therefore implies that $\frac{\partial T_{s}}{\partial s}(0) \neq 0$. Let us define

$$
\chi(y):=\operatorname{sign}\left(-\frac{\partial T_{s}}{\partial s}(0)\right) .
$$


Now suppose $(x, \eta) \in \operatorname{Crit}\left(A_{H-k}\right)$ with $\eta>0$. Let $y: \mathbb{R} / \eta \mathbb{Z} \rightarrow \Sigma_{k}$ be defined by $y(t):=x(t / \eta)$. Define

$$
\chi(x, \eta):=\chi(y)
$$

If $(x, \eta) \in \operatorname{Crit}\left(A_{H-k}\right)$ with $\eta<0$ define

$$
\chi(x, \eta):=-\chi\left(x^{-},-\eta\right)
$$

where $x^{-}(t):=x(-t)$ (note that $\left(x^{-},-\eta\right) \in \operatorname{Crit}\left(A_{H-k}\right)$, so this makes sense).

Thus

$$
\chi(q, T)=\chi\left(Z^{+}(q, T)\right)=-\chi\left(Z^{-}(q, T)\right) .
$$

for any $(q, T) \in \operatorname{Crit}\left(S_{L+k}\right)$, where $\chi(q, T)$ is defined as in $(3.5)$.

We define a grading $\mu: \operatorname{Crit}\left(A_{H-k}\right) \rightarrow \mathbb{Z}$ on $\operatorname{Crit}\left(A_{H-k}\right)$ as follows.

4.3. Definition. Given $(x, \eta) \in \operatorname{Crit}\left(A_{H-k}\right)$ with $\eta \neq 0$ define $y: \mathbb{R} /|\eta| \mathbb{Z} \rightarrow \Sigma_{k}$ by $y(t):=$ $x(t /|\eta|)$. Then $y$ is an $|\eta|$-periodic orbit of $\operatorname{sign}(\eta) H$. Let us denote by $\mu_{\mathrm{CZ}}(y)$ the Conley-Zehnder index of y. See [46] for the definition of the Conley-Zehnder index in the degenerate case that we are using (note however that our sign conventions match those of [2] not [46]). Define

$$
\mu(x, \eta):= \begin{cases}\mu_{\mathrm{CZ}}(y)-\frac{1}{2} \chi(x, \eta) & \eta \neq 0 \\ -n+1 & \eta=0 .\end{cases}
$$

We wish to compare $\mu\left(Z^{ \pm}(q, T)\right)$ with $i(q, T)$ for $(q, T) \in \operatorname{Crit}\left(S_{L+k}\right)$. We will need an extension of the Morse index theorem of Duistermaat [25] to the twisted symplectic form $\omega$ :

4.4. TheOREm. Let $(q, T) \in \operatorname{Crit}\left(S_{L+k}\right)$. Let $y: \mathbb{R} / T \mathbb{Z} \rightarrow \Sigma$ be defined by $y(t):=Z^{+}(q, T)(t / T)$. Then

$$
\mu_{\mathrm{CZ}}(y)-\frac{1}{2}=i_{T}(q) \text {. }
$$

Proof. We deduce this from the equivalent statement for the standard symplectic form $\omega_{0}$ (specifically, from [2, Corollary 4.2]) by arguing as follows: take a tubular neighborhood $W$ of $q\left(S^{1}\right)$ in $M$. Since $H^{2}(W)=0,\left.\sigma\right|_{W}=d \theta$ for some $\theta \in \Omega^{1}(W)$. The flow $\left.\phi_{t}^{H}\right|_{W}$ is conjugated to the flow $\psi_{t}^{H_{\theta}}$ : $T^{*} W \rightarrow T^{*} W$, where $H_{\theta}(q, p)=H\left(q, p-\theta_{q}\right)$ and $\psi_{t}^{H_{\theta}}$ denotes the flow of the symplectic gradient of $H_{\theta}$ with respect to the standard symplectic form $\omega_{0}$. Since both the Maslov index and the Morse index are local invariants, the theorem now follows directly from [2, Corollary 4.2].

4.5. REMARK. In [40] we provide a direct proof of Theorem 4.4] based on Weber's proof [48, Theorem 1.3] of the corresponding statement for the standard symplectic form.

The next corollary is an immediate consequence of Theorems 3.5 and 4.4, and the definition of the Conley-Zehnder index.

4.6. Corollary. Let $(q, T) \in \operatorname{Crit}\left(S_{L+k}\right)$. Then

$$
\mu\left(Z^{ \pm}(q, T)\right)= \pm i(q, T) .
$$

\subsection{The moduli spaces of Rabinowitz Floer homology.}

Throughout this subsection assume $(g, \sigma, U, k) \in \mathcal{O}_{\text {reg }}$ is fixed (recall by assumption this means $k>c(g, \sigma, U)$, cf. Definition 2.1), and put $H=H_{g}+\pi^{*} U$. Fix $J \in \mathcal{J}(\omega)$. We are interested in maps $u: \mathbb{R} \rightarrow \Lambda T^{*} M \times \mathbb{R}$ that satisfy the Rabinowitz Floer equation:

$$
u^{\prime}(s)+\nabla A_{H-k}(u(s))=0
$$

together with the asymptotic conditions

$$
\lim _{s \rightarrow \pm \infty} u(s) \in \operatorname{Crit}\left(A_{H-k}\right) .
$$


It is well known that any such map $u$ is smooth, and extends to a map (also denoted by) $u: \overline{\mathbb{R}} \rightarrow$ $C^{\infty}\left(S^{1}, T^{*} M\right) \times \mathbb{R}$. We shall often regard such a map $u$ as an element of $C^{\infty}\left(\mathbb{R} \times S^{1}, T^{*} M\right) \times$ $C^{\infty}(\mathbb{R}, \mathbb{R})$. If we write $u(s, t)=(x(s, t), \eta(s))$ then (4.3) implies that $x$ and $\eta$ solve the coupled equations

$$
\begin{gathered}
x^{\prime}+J(t, x)\left(\dot{x}-\eta X_{H}(x)\right)=0 ; \\
\eta^{\prime}-\int_{S^{1}}(H(x(t))-k) d t=0 .
\end{gathered}
$$

Choose a Morse function $h: \operatorname{Crit}\left(A_{H-k}\right) \rightarrow \mathbb{R}$ and a Riemannian metric $g_{1}$ on $\operatorname{Crit}\left(A_{H-k}\right)$ such that the negative gradient flow $\phi_{t}^{-\nabla h}$ of $-\nabla h$ is Morse-Smale. Denote by $\operatorname{Crit}(h) \subseteq \operatorname{Crit}\left(A_{H-k}\right)$ the set of critical points of $h$. The Morse-Smale assumption implies that for every pair $z_{-}, z_{+}$of critical points of $h$ the unstable manifold $W^{u}\left(z_{-} ;-\nabla h\right)$ intersects the stable manifold $W^{s}\left(z_{+} ;-\nabla h\right)$ transversely. Denote by $i_{h}(z):=\operatorname{dim} W^{u}(z ;-\nabla h)$ the Morse index of a critical point $z \in \operatorname{Crit}(h)$. We define a new grading $\widehat{\mu}_{h}: \operatorname{Crit}(h) \rightarrow \mathbb{Z}$ by putting

$$
\widehat{\mu}_{h}(z):=\mu(z)+i_{h}(z) .
$$

Suppose $z_{ \pm}=\left(x_{ \pm}, \eta_{ \pm}\right) \in \operatorname{Crit}(h)$ are critical points of $h$. Denote by

$$
\widetilde{\mathcal{M}}_{0}\left(z_{-}, z_{+}\right):=W^{u}\left(z_{-} ;-\nabla h\right) \cap W^{s}\left(z_{+} ;-\nabla h\right) .
$$

Let

$$
\mathcal{M}_{0}\left(z_{-}, z_{+}\right):=\widetilde{\mathcal{M}}_{0}\left(z_{-}, z_{+}\right) / \mathbb{R}
$$

denote the quotient of $\widetilde{\mathcal{M}}_{0}\left(z_{-}, z_{+}\right)$by the obvious free $\mathbb{R}$-action (if $z_{-}=z_{+}, \mathcal{M}_{0}\left(z_{-}, z_{+}\right)=\emptyset$ ). Given $m \in \mathbb{N}$, let

$$
\widetilde{\mathcal{M}}_{m}\left(z_{-}, z_{+}\right)
$$

denote the set of tuples of maps $\boldsymbol{u}=\left(u_{1}, \ldots, u_{m}\right)$ such that each $u_{i}: \mathbb{R} \rightarrow C^{\infty}\left(S^{1}, T^{*} M\right) \times \mathbb{R}$ satisfies the Rabinowitz Floer equation (4.3) and is non-stationary (here a stationary solution is one that does not depend on $s$ ) and such that

$$
\begin{gathered}
u_{1}(-\infty) \in W^{u}\left(z_{-} ;-\nabla h\right), \ldots, u_{m}(\infty) \in W^{s}\left(z_{+} ;-\nabla h\right) ; \\
u_{i+1}(-\infty) \in \phi_{\mathbb{R}^{+}}^{-\nabla h}\left(u_{i}(\infty)\right) .
\end{gathered}
$$

Note that if $m \geq 1$ then $\widetilde{\mathcal{M}}_{m}\left(z_{-}, z_{+}\right)$admits a free action of $\mathbb{R}^{m}$ via

$$
\left(u_{1}(s), \ldots, u_{m}(s)\right) \mapsto\left(u_{1}\left(s+s_{1}\right), \ldots, u_{m}\left(s+s_{m}\right)\right), \quad\left(s_{1}, \ldots, s_{m}\right) \in \mathbb{R}^{m} .
$$

We denote by $\mathcal{M}_{m}\left(z_{-}, z_{+}\right)$the quotient of $\widetilde{\mathcal{M}}_{m}\left(z_{-}, z_{+}\right)$by this action. Put

$$
\mathcal{M}\left(z_{-}, z_{+}\right):=\bigcup_{m \in \mathbb{N} \cup\{0\}} \mathcal{M}_{m}\left(z_{-}, z_{+}\right) .
$$

Since $A_{H-k}$ is strictly decreasing on non-stationary solutions of the Rabinowitz Floer equation, if $z_{-}$and $z_{+}$belong to the same connected component of $\operatorname{Crit}\left(A_{H-k}\right)$ then $\mathcal{M}_{m}\left(z_{-}, z_{+}\right)=\emptyset$ for all $m \geq 1$, and if $\mathcal{M}_{m}\left(z_{-}, z_{+}\right) \neq \emptyset$ for some $m \geq 1$, then $A_{H-k}\left(z_{-}\right)>A_{H-k}\left(z_{+}\right)$and $\mathcal{M}_{0}\left(z_{-}, z_{+}\right)=\emptyset$.

The central result we need to construct the Rabinowitz Floer complex is the following:

4.7. THEOREM. There exists $\varepsilon_{1}>0$ such that if $J \in \mathcal{J}(\omega) \cap B_{\varepsilon_{1}}\left(J_{g}\right)$ is a generically chosen almost complex structure and $g_{1}$ is a generically chosen Morse-Smale metric for $h$ then the moduli spaces $\mathcal{M}\left(z_{-}, z_{+}\right)$are all finite dimensional smooth manifolds, and their components of dimension zero are compact. Moreover we have

$$
\operatorname{dim} \mathcal{M}\left(z_{-}, z_{+}\right)=\widehat{\mu}_{h}\left(z_{-}\right)-\widehat{\mu}_{h}\left(z_{+}\right)-1 .
$$

The proof of the theorem has four ingredients: 
(1) Exhibit $\mathcal{M}\left(z_{-}, z_{+}\right)$as the zero set of a certain section of a Banach bundle.

(2) Show that the linearization of this operator is Fredholm, and compute its index.

(3) Show that for generic $J, g_{1}$ the linearization is surjective.

(4) Exhibit uniform $C_{\mathrm{loc}}^{\infty}$ bounds for gradient flow lines.

We refer to one of the many references (perhaps the two most relevant are [28, Appendix A] and [3, Section 3]) as to why solving these four problems does indeed lead to a proof of the theorem. Problem (1) was solved in [28, Appendix A]. Problem (2) was solved for defining Hamiltonians and restricted contact type hypersurfaces in [16, Section 4]. In our situation there is an additional complication in computing the indices (stemming from the correction term $-\frac{1}{2} \chi(z)$ ). Full details of the computation of the index are contained in [41]. Alternatively one could probably use the methods of [14, Section 3.2].

Problem (3) can be solved using the methods in [27] combined with the Morse-Bott formalism of [28, Theorem A.14]. Problem (4) was solved for Hamiltonians that are constant outside a compact set in [16, Section 3] and extended to Hamiltonians that are linear at infinity [19, Section 5] and then Hamiltonians which grow quadratically and radially at infinity [4, Section 2]. None of these are applicable for the Hamiltonians $H_{g}+\pi^{*} U$ that we consider, and hence we will give a complete proof of this below. Our methods are essentially those of [3]. Since $\left.\omega\right|_{\pi_{2}(M)}=0$ and $c_{1}\left(T^{*} M, \omega\right)=0$, in order to get $C_{\mathrm{loc}}^{\infty}$ bounds on gradient flow lines of the Rabinowitz Floer equation it is sufficient to obtain $L^{\infty}$ bounds (in short, this is because the so-called 'bubbling' phenomenon cannot occur). Obtaining these $L^{\infty}$ estimates is the subject of Subsection 4.6 below.

4.8. REMARK. It is perhaps useful to explain exactly where our various hypotheses are used. The fact that $(g, \sigma, U, k) \in \mathcal{O}$ (i.e. $k>c(g, \sigma, U))$ is used in order in order to obtain $L^{\infty}$ bounds on the $\eta$-component of gradient flows lines $u \in \mathcal{M}\left(z_{-}, z_{+}\right)$. The bound on the $x$-component requires two assumptions: firstly that the $\eta$-component is uniformly bounded, and secondly that $J \in B_{\varepsilon_{1}}\left(J_{g}\right)$. Finally, the assumption $(g, \sigma, U, k) \in \mathcal{O}_{\text {reg }}$ is used in order to compute the index of the operator defining the moduli space $\mathcal{M}\left(z_{-}, z_{+}\right)$- recall that our grading $\mu$ explicitly used the existence of an orbit cylinder, which need not exist if only $(g, \sigma, U, k) \in \mathcal{O}$.

4.9. REMARK. The constant $\varepsilon_{1}>0$ appearing in the statement of Theorem 4.7 is a universal constant (cf. Theorem 4.13 below). In order for the statement of Theorem 4.7 not to be completely vacuous one of course needs to know that such almost complex structures exist. This can be guaranteed by assuming $\|\sigma\|$ is sufficiently small. Indeed, suppose $\sigma$ satisfies

$$
\|\sigma\|_{\infty} \leq \frac{\varepsilon_{1}}{2 \varepsilon_{0}}
$$

Then by (2.3) we have

$$
B_{\varepsilon_{1} / 2}\left(J_{\sigma}\right) \subseteq B_{\varepsilon_{1}}\left(J_{g}\right)
$$

\subsection{Constructing the chain complex.}

Deferring the proof of Problem (4), we first explain the construction of Rabinowitz Floer chain complex. Assume that the hypotheses of Theorem 4.7 are satisfied. Denote by $R F\left(A_{H-k}, h\right)$ the $\mathbb{Z}_{2}$-vector space generated by all formal sums

$$
\sum_{z \in V} z
$$

where $V \subseteq \operatorname{Crit}(h)$ is a (possibly infinite) subset of Crit $(h)$ satisfying the Novikov finiteness condition that for all $a \in \mathbb{R}$ one has

$$
\#\left\{z \in V: A_{H-k}(z)<a\right\}<\infty .
$$

Let us write $\operatorname{Crit}_{i}(h) \subseteq \operatorname{Crit}(h)$ for the set of critical points $z$ of $h$ with $\widehat{\mu}_{h}(z)=i$. The vector space $R F\left(A_{H-k}, h\right)$ is given a $\mathbb{Z}$-grading by the index $\widehat{\mu}_{h}$ : an element $\sum_{z \in V} z \in R F\left(A_{H-k}, h\right)$ belongs to 
$R F_{i}\left(A_{H-k}, h\right)$ if $V \subseteq \operatorname{Crit}_{i}(h)$. Similarly, given an interval $(a, b) \subseteq \overline{\mathbb{R}}$, denote by $R F^{(a, b)}\left(A_{H-k}, h\right)$ the $\mathbb{Z}_{2}$-vector space of all formal sums

$$
\sum_{z \in V} z
$$

where $V \subseteq \mathrm{Crit}^{(a, b)}(h)$ is a (possibly infinite) subset of Crit ${ }^{(a, b)}(h)$ satisfying the finiteness condition above (note that if $a$ and $b$ are finite then such a set $V$ is necessarily finite and the Novikov finiteness condition is automatic).

If $z_{ \pm} \in \operatorname{Crit}(h)$ satisfy $\widehat{\mu}_{h}\left(z_{-}\right)-\widehat{\mu}_{h}\left(z_{+}\right)=1$ then Theorem 4.7 tells us that $\mathcal{M}\left(z_{-}, z_{+}\right)$is a finite set. We can therefore define $n_{\text {Rab }}\left(z_{-}, z_{+}\right)$by

$$
n_{\mathrm{Rab}}\left(z_{-}, z_{+}\right):=\# \mathcal{M}\left(z_{-}, z_{+}\right), \text {taken } \bmod 2 .
$$

Then we define

$$
\partial^{\mathrm{Rab}}=\partial^{\mathrm{Rab}}\left(J, g_{1}\right): R F_{i}\left(A_{H-k}, h\right) \rightarrow R F_{i-1}\left(A_{H-k}, h\right)
$$

by

$$
\partial^{\mathrm{Rab}} z:=\sum_{z^{\prime} \in \mathrm{Crit}_{i-1}(h)} n_{\mathrm{Rab}}\left(z, z^{\prime}\right) z^{\prime},
$$

and extending by linearity. A standard gluing argument tells us that $\partial^{\mathrm{Rab}} \circ \partial^{\mathrm{Rab}}=0$, and therefore we conclude that $\left\{R F_{*}\left(A_{H-k}, h\right), \partial^{\mathrm{Rab}}\left(J, g_{1}\right)\right\}$ is a chain complex of Abelian groups. The boundary map $\partial^{\mathrm{Rab}}$ respects the $\mathbb{R}$-filtration determined by $A_{H-k}$ : if $(a, b) \subseteq \overline{\mathbb{R}}$ then

$$
\partial^{\operatorname{Rab}}\left(R F_{i}^{(a, b)}\left(A_{H-k}, h\right)\right) \subseteq R F_{i-1}^{(a, b)}\left(A_{H-k}, h\right),
$$

and so $\left\{R F_{*}^{(a, b)}\left(A_{H-k}, h\right), \partial^{\mathrm{Rab}}\left(J, g_{1}\right)\right\}$ is a subcomplex. Finally it is clear that $\partial^{\mathrm{Rab}}$ also respects the splitting $\Lambda T^{*} M \oplus \mathbb{R}=\bigoplus_{\alpha \in\left[S^{1}, M\right]} \Lambda_{\alpha} T^{*} M \times \mathbb{R}$ : if $R F_{*}\left(A_{H-k}, h ; \alpha\right)$ denotes the subspace of $R F_{*}\left(A_{H-k}, h\right)$ generated by the elements of $\operatorname{Crit}(h) \cap \operatorname{Crit}\left(A_{H-k} ; \alpha\right)$ then $R F_{*}\left(A_{H-k}, h ; \alpha\right)$ is a subcomplex.

We write $R F H_{*}\left(A_{H-k}\right)$ for the homology of $\left\{R F_{*}\left(A_{H-k}, a\right), \partial^{\mathrm{Rab}}\left(J, g_{1}\right)\right\}$ and call it the Rabinowitz Floer homology of $A_{H-k}$. Similarly we write $R F H_{*}\left(A_{H-k} ; \alpha\right)$ (resp. $R F H_{*}^{(a, b)}\left(A_{H-k}\right)$ ) for the homology of the subcomplex $R F_{*}\left(A_{H-k}, h ; \alpha\right)$ (resp. $R F_{*}^{(a, b)}\left(A_{H-k}, h\right)$ ). Standard arguments show that $R F H_{*}\left(A_{H-k}\right)$ is independent of the data $\left(h, J, g_{1}\right)$.

4.10. REMARK. In fact, if $\left(g_{s}, \sigma_{s}, U_{s}, k_{s}\right)_{s \in[0,1]} \subseteq \mathcal{O}$ is a smooth family that satisfies $\left(g_{s}, \sigma_{s}, U_{s}, k_{s}\right) \in$ $\mathcal{O}_{\text {reg }}$ for generic $s \in[0,1]$ and in particular for $s=0,1$ then if $H_{s}(q, p):=\frac{1}{2}|p|_{g_{s}}^{2}+U_{s}(q)$ and $\omega_{s}:=\omega_{0}+\pi^{*} \sigma_{s}$ then $R F H_{*}\left(A_{H_{0}-k_{0}} ; \omega_{0}\right) \cong R F H_{*}\left(A_{H_{1}-k_{1}} ; \omega_{1}\right)$. One can prove this directly using the methods of [3, Section 1.8] and [12]. However we can deduce this indirectly via Theorem 1.1.(b) in [20] and Theorem 1.4 in [12], by making use of Proposition 6.2 below, which states that the Rabinowitz Floer homology $R F H_{*}\left(A_{H-k}\right)$ is the same as the Rabinowitz Floer homology $R F H_{*}\left(\Sigma_{k}, T^{*} M\right)$ from [20].

As a consequence we are free to define the Rabinowitz Floer homology $R F H_{*}\left(A_{H-k}\right)$ for the Hamiltonian $H=H_{g}+\pi^{*} U$ if only $(g, \sigma, U, k) \in \mathcal{O}$ (rather than $(g, \sigma, U, k) \in \mathcal{O}_{\text {reg }}$ ). Indeed, by Theorem 3.4 we can find a metric $g^{\prime}$ lying arbitrarily close to $g$ such that $(g, \sigma, U, k) \in \mathcal{O}_{\text {reg. }}$. Set $H^{\prime}:=H_{g^{\prime}}+\pi^{*} U$ and define $R F H_{*}\left(A_{H-k}\right):=R F H_{*}\left(A_{H^{\prime}-k}\right)$. This is well defined, as if $g^{\prime \prime}$ is another such metric and $H^{\prime \prime}:=H_{g^{\prime \prime}}+\pi^{*} U$ then the previous paragraph implies $R F H_{*}\left(A_{H^{\prime}-k}\right) \cong R F H_{*}\left(A_{H^{\prime \prime}-k}\right)$.

\subsection{The $L^{\infty}$ estimates.}

In this subsection we prove the two theorems on $L^{\infty}$ estimates for solutions of the Rabinowitz Floer equation alluded to above, as well as a third $L^{\infty}$ estimate for gradient flow lines defined on halfcylinders that will be needed in the next section. The first result we state is an extension of part of 
[16. Theorem 3.1], which obtains uniform $L^{\infty}$ bounds for the $\eta$-component of flow lines $u=(x, \eta) \in$ $C^{\infty}\left(\mathbb{R} \times S^{1}, T^{*} M\right) \times C^{\infty}(\mathbb{R}, \mathbb{R})$ satisfying the Rabinowitz Floer equation and having bounded $A_{H-k^{-}}$ action. This result (for contractible loops only) was stated without proof in [20, Section 7].

4.11. TheOREM. Let $(g, \sigma, U, k) \in \mathcal{O}$ and put $H=H_{g}+\pi^{*} U$. Pick $J \in \mathcal{J}(\omega)$ and $\alpha \in\left[S^{1}, M\right]$, and fix $-\infty<a<b<\infty$. There exists a constant $C_{0}>0$ such that if $u=(x, \eta) \in C^{\infty}\left(\mathbb{R} \times S^{1}, T^{*} M\right) \times$ $C^{\infty}(\mathbb{R}, \mathbb{R})$ is any map that satisfies the Rabinowitz Floer equation (4.3) and has action bounds

$$
A_{H-k}(u(\mathbb{R})) \subseteq[a, b]
$$

and satisfies

$$
x(\mathbb{R}, \cdot) \in \Lambda_{\alpha} T^{*} M
$$

then

$$
\|\eta\|_{L^{\infty}(\mathbb{R})} \leq C_{0}
$$

4.12. REMARK. We emphasize that the following proof uses only that $\Sigma_{k}:=H^{-1}(k)$ is of virtual restricted contact type (see [20, p1767] for the definition) for $k>c(g, \sigma, U)$; it makes no assumptions on the behaviour of the Hamiltonian $H$ at infinity. In other words, the proof would go through if instead of $H$ we used any other Hamiltonian $K \in C^{\infty}\left(T^{*} M, \mathbb{R}\right)$ with the property that $X_{K}\left|\Sigma_{k}=f X_{H}\right|_{\Sigma_{k}}$ for some smooth function $f \in C^{\infty}\left(\Sigma_{k}, \mathbb{R}^{+}\right)$.

Proof. (of Theorem 4.11)

The proof is a slight modification of the arguments of [16, Section 3]. Let $\widetilde{H}: T^{*} \widetilde{M} \rightarrow \mathbb{R}$ denote the lift of $H$ to $\widetilde{\pi}: T^{*} \widetilde{M} \rightarrow \widetilde{M}$. Let $\widetilde{\omega}:=\widetilde{\omega}_{0}+\widetilde{\pi}^{*} \widetilde{\sigma}$, where $\widetilde{\omega}_{0}=d \widetilde{\lambda}_{0}$ is the canonical symplectic form on $T^{*} \widetilde{M}$. Since $k>c(g, \sigma, U)$, by [20, Lemma 5.1] there exists a primitive $\theta$ of $\widetilde{\sigma}$ and $\delta>0$ such that

$$
\widetilde{\lambda}\left(X_{\widetilde{H}}(x)\right) \geq 2 \delta \text { for all } x \in \widetilde{H}^{-1}([k-\delta, k+\delta]) .
$$

Here

$$
\widetilde{\lambda}:=\widetilde{\lambda}_{0}+\widetilde{\pi}^{*} \theta,
$$

and $X_{\widetilde{H}}$ is the symplectic gradient of the lifted function $\widetilde{H}$ with respect to the symplectic form $\widetilde{\omega}=d \widetilde{\lambda}$. Observe that it follows from (3.2) that for any $x \in \Lambda_{\alpha} T^{*} M$ and any lift $\widetilde{x}:[0,1] \rightarrow T^{*} \widetilde{M}$ we have

$$
\int_{C} \bar{x}^{*} \omega=\int_{0}^{1} \widetilde{x}^{*} \widetilde{\lambda}+I(\alpha, \theta) .
$$

The first part of the proof is the following statement: there exists a constant $\rho_{0}=\rho(\delta)>0$ such that:

$$
\left\|\nabla A_{H-k}(x, \eta)\right\|_{J} \leq \rho_{0} \quad \Rightarrow \quad x\left(S^{1}\right) \subseteq H^{-1}([k-\delta, k+\delta])
$$

(where $\delta>0$ is the constant from (4.6)). This part of the proof is identical to [16, Proposition 3.2, Step 2], and hence is omitted.

Next we show that there exists a constant $D<\infty$ such that if $(x, \eta) \in \Lambda_{\alpha} T^{*} M \times \mathbb{R}$ is any loop that satisfies

$$
x\left(S^{1}\right) \subseteq H^{-1}([k-\delta, k+\delta]),
$$

(where $\delta>0$ is the constant from (4.6)) then

$$
|\eta|<\frac{1}{\delta}\left|A_{H-k}(x, \eta)\right|+\frac{D}{\delta}\left\|\nabla A_{H-k}(x, \eta)\right\|_{J}+\frac{1}{\delta}|I(\alpha, \theta)| .
$$

Indeed, set

$$
D:=\left\|\left.\widetilde{\lambda}\right|_{\widetilde{H}^{-1}([k-\delta, k+\delta])}\right\|_{\infty},
$$


and compute using using (4.7):

$$
\begin{aligned}
\left|A_{H-k}(x, \eta)\right| & =\left|\int_{C} \bar{x}^{*} \omega-\eta \int_{S^{1}}(H(x(t))-k) d t\right| \\
& \geq\left|\int_{0}^{1} \widetilde{\lambda}(\dot{\widetilde{x}}) d t\right|-|I(\alpha, \theta)|-|\eta|\left|\int_{\mathbb{T}}(H(x(t))-k) d t\right| \\
& \geq\left|\int_{0}^{1} \widetilde{\lambda}\left(\eta X_{\widetilde{H}}(\widetilde{x})\right) d t\right|-\left|\int_{0}^{1} \widetilde{\lambda}\left(\dot{\widetilde{x}}-\eta X_{\widetilde{H}}(\widetilde{x})\right) d t\right|-|I(\alpha, \theta)|-|\eta| \delta . \\
& \geq|\eta|(2 \delta-\delta)-D \int_{S^{1}}\left|\dot{x}-\eta X_{H}(x)\right| d t-|I(\alpha, \theta)| \\
& \geq|\eta| \delta-D\left\|\nabla A_{H-k}(x, \eta)\right\|_{J}-|I(\alpha, \theta)| .
\end{aligned}
$$

This proves (4.9). Combining (4.8) and (4.9) we see that if

$$
\rho_{1}:=\frac{1}{\delta} \max \left\{1, D \rho_{0}+|I(\alpha, \theta)|\right\}
$$

then the following implication holds: for any $(x, \eta) \in \Lambda_{\alpha} T^{*} M \times \mathbb{R}$,

$$
\left\|\nabla A_{H-k}(x, \eta)\right\|_{J} \leq \rho_{0} \Rightarrow|\eta| \leq \rho_{1}\left(A_{H-k}(x, \eta)+1\right) .
$$

We can now prove the theorem. Let $u=(x, \eta) \in C^{\infty}\left(\mathbb{R} \times S^{1}, T^{*} M\right) \times C^{\infty}(\mathbb{R}, \mathbb{R})$ satisfy the hypotheses of the theorem. Given $s \in \mathbb{R}$ let

$$
\tau(s):=\inf \left\{r \geq 0:\left\|\nabla A_{H-k}(u(s+r, \cdot))\right\|_{J} \leq \rho_{0}\right\} .
$$

Then for any $s \in \mathbb{R}$ we have:

$$
\begin{aligned}
b-a & \geq \int_{-\infty}^{\infty}\left\|\nabla A_{H-k}(u(r, \cdot))\right\|_{J}^{2} d r \\
& \geq \int_{s}^{s+\tau(s)}\left\|\nabla A_{H-k}(u(r, \cdot))\right\|_{J}^{2} d r \\
& \geq \tau(s) \rho_{0}^{2},
\end{aligned}
$$

and hence

$$
\tau(s) \leq \frac{b-a}{\rho_{0}^{2}}
$$

Thus given any $s \in \mathbb{R}$ we have

$$
\begin{aligned}
|\eta(s)| & =\left|\eta(s+\tau(s))-\int_{s}^{s+\tau(s)} \eta^{\prime}(r) d t\right| \\
& \leq \rho_{1}\left(\left|A_{H-k}(u(s+\tau(s), \cdot))\right|+1\right)+\int_{s}^{s+\tau(s)}\left|\eta^{\prime}(r)\right| d r \\
& \leq \rho_{1}(\max \{|a|,|b|\}+1)+\left(\tau(s) \int_{s}^{s+\tau(s)}\left|\eta^{\prime}(r)\right|^{2} d s\right)^{1 / 2} \\
& \leq \rho_{1}(\max \{|a|,|b|\}+1)+\left(\frac{b-a}{\rho_{0}^{2}} \int_{s}^{s+\tau(s)}\left\|u^{\prime}(r, \cdot)\right\|_{J}^{2} d s\right)^{1 / 2} \\
& \leq \rho_{1}(\max \{|a|,|b|\}+1)+\frac{b-a}{\rho_{0}} .
\end{aligned}
$$


Thus the theorem follows with

$$
C_{0}:=\rho_{1}(\max \{|a|,|b|\}+1)+\frac{b-a}{\rho_{0}} .
$$

In the next result we are interested in obtaining bounds on the loop component $x$ of a flow line $u$. The proof uses the same idea as [3, Theorem 1.14, Theorem 1.22], and is based upon isometrically embedding $(M, g)$ into Euclidean space, and combining Calderon-Zygmund estimates for the CauchyRiemann operator with certain interpolation inequalities. In the course of the proof we will need the following statement, which is a consequence of the Calderon-Zygmund inequalities. Let

$$
W_{V}^{1, r}\left(\mathbb{R} \times S^{1}, \mathbb{R}^{2 d}\right)=W_{0}^{1, r}\left(\mathbb{R} \times S^{1}, \mathbb{R}^{d}\right) \times W^{1,3}\left(\mathbb{R} \times S^{1}, \mathbb{R}^{d}\right)
$$

denote the Sobolev space of $\mathbb{R}^{2 d}$-valued maps taking values in the vertical Lagrangian subspace $V:=$ (0) $\times \mathbb{R}^{d} \subseteq \mathbb{R}^{2 d}$ on the boundary.

4.13. THEOREM. Let $J_{0}$ denote the standard complex structure on $\mathbb{R}^{2 d}$ given by

$$
J_{0}=\left(\begin{array}{cc}
0 & -\mathbb{1} \\
\mathbb{1} & 0
\end{array}\right)
$$

\section{Consider the Cauchy-Riemann operator}

$$
\partial_{s}+J_{0} \partial_{t}: W_{V}^{1,3}\left(\mathbb{R} \times S^{1}, \mathbb{R}^{2 d}\right) \rightarrow L^{3}\left(\mathbb{R} \times S^{1}, \mathbb{R}^{2 d}\right) .
$$

Then there exists a constant $\varepsilon_{1}>0$ such that for any $v \in W_{V}^{1,3}\left(\mathbb{R} \times S^{1}, \mathbb{R}^{2 d}\right)$ it holds that

$$
\|\nabla v\|_{L^{3}\left(\mathbb{R} \times S^{1}\right)} \leq \frac{1}{2 \varepsilon_{1}}\left\|\left(\partial_{s}+J_{0} \partial_{t}\right) v\right\|_{L^{3}\left(\mathbb{R} \times S^{1}\right)} .
$$

We now prove:

4.14. TheOREM. Fix $(g, \sigma, U, k) \in \mathcal{O}$. Suppose $J \in \mathcal{J}(\omega) \cap B_{\varepsilon_{1}}\left(J_{g}\right)$ (where $\varepsilon_{1}>0$ is as in Theorem 4.13), $\alpha \in\left[S^{1}, M\right]$ and $-\infty<a<b<\infty$. Put $H=H_{g}+\pi^{*} U$. Assume there exists a constant $C_{0}>0$ such that if $u=(x, \eta) \in C^{\infty}\left(\mathbb{R} \times S^{1}, T^{*} M\right) \times C^{\infty}(\mathbb{R}, \mathbb{R})$ is any map that satisfies the Rabinowitz Floer equation (4.3) and has action bounds

$$
A_{H-k}(u(\mathbb{R})) \subseteq[a, b]
$$

and satisfies

$$
x(\mathbb{R}, \cdot) \in \Lambda_{\alpha} T^{*} M
$$

then

$$
\|\eta\|_{L^{\infty}(\mathbb{R})} \leq C_{0} .
$$

Then there exists another constant $C_{1}>0$ such that for any such map $u=(x, \eta)$ it also holds that

$$
\|x\|_{L^{\infty}(\mathbb{R})}<C_{1} .
$$

In the proof below we will repeatedly use the fact there exists a constant $b_{0}>0$ such that

$$
\left|X_{H}(q, p)\right| \leq b_{0}\left(1+|p|^{2}\right) \quad \text { for all }(q, p) \in T^{*} M .
$$

Proof. (of Theorem 4.14)

We begin by choosing an isometric embedding of $i:(M, g) \rightarrow\left(\mathbb{R}^{d}, g_{0}\right)$, where $g_{0}$ is the Euclidean inner product. Such an embedding exists by Nash's theorem. It induces an embedding (also denoted by) $i: T^{*} M \rightarrow \mathbb{R}^{2 d}$ which is actually a unitary embedding (with respect to the standard symplectic form), that is,

$$
i^{*} \omega_{0}=\omega_{0}, \quad i_{*} J_{0}=J_{g},
$$


where

$$
J_{0}=\left(\begin{array}{cc}
0 & -\mathbb{1} \\
\mathbb{1} & 0
\end{array}\right)
$$

is the standard almost complex structure on $\mathbb{R}^{2 d}$. Thus under this embedding the metric almost complex structure $J_{g}(2.2)$ is simply the restriction of the canonical almost complex structure $J_{0}$ to $T^{*} M$, and hence the assumption that $J \in \mathcal{J}(\omega) \cap B_{\varepsilon_{1}}\left(J_{g}\right)$ corresponds to $J \in \mathcal{J}(\omega) \cap B_{\varepsilon_{1}}\left(J_{0}\right)$. We will use this embedding to define the various $L^{r}$ and $W^{1, r}$ spaces that come up in the proof below.

The proof of the theorem is in two steps.

\section{Step 1.}

We show that there exists a constant $K>0$ such that for any map $u=(x, \eta)$ satisfying the hypotheses of the theorem, and any finite interval $I \subseteq \mathbb{R}$, writing $x=(q, p)$ it holds that

$$
\|p\|_{L^{2}\left(I \times S^{1}\right)} \leq K|I|^{1 / 2}, \quad\|\nabla p\|_{L^{2}\left(I \times S^{1}\right)} \leq K\left(1+|I|^{1 / 2}\right) .
$$

This part of the proof closely follows [3, Lemma 1.12], and heavily uses the fact that our Hamiltonian $H$ is quadratic. This step does not use the fact that $J \in B_{\varepsilon_{1}}\left(J_{0}\right)$.

We first note that there exists a constant $b_{1}>0$ such that for any map $u=(x, \eta)$ satisfying the hypotheses of the theorem,

Indeed, if $s_{0}<s_{1}$ then

$$
\left\|x^{\prime}\right\|_{L^{2}\left(\mathbb{R} \times S^{1}\right)} \leq b_{1}, \quad\left\|\eta^{\prime}\right\|_{L^{2}(\mathbb{R})} \leq b_{1}
$$

$$
\begin{aligned}
\left\|x^{\prime}\right\|_{L^{2}\left(\left(s_{0}, s_{1}\right) \times S^{1}\right)}^{2} & =\int_{s_{0}}^{s_{1}} \int_{S^{1}}\left|x^{\prime}\right|^{2} d t d s \\
& \leq\left\|J^{-1}\right\|_{\infty}^{2} \int_{s_{0}}^{s_{1}} \int_{S^{1}}\left\|u^{\prime}\right\|_{J}^{2} d t d s \\
& \leq\|J\|_{\infty}^{2}(b-a) .
\end{aligned}
$$

Exactly the same computation holds for $\left\|\eta^{\prime}\right\|_{L^{2}(\mathbb{R})}$, and hence we may take

$$
b_{1}:=\|J\|_{\infty} \sqrt{b-a} .
$$

We next claim that there exists a constant $b_{2}>0$ such that for any finite interval $I \subseteq \mathbb{R}$ and for any map $u=(x, \eta)$ satisfying the hypotheses of the theorem, if we write $x=(q, p)$, then

$$
\|p\|_{L^{2}\left(I \times S^{1}\right)}^{2} \leq b_{2} \max \left\{|I|,|I|^{1 / 2}\right\} .
$$

Indeed,

$$
\begin{aligned}
\eta^{\prime}(s) & =\int_{S^{1}}(H(x(s, t))-k) d t \\
& \geq \int_{S^{1}} \frac{1}{2}|p(s, t)|^{2} d t-\left(\|U\|_{\infty}+k\right) .
\end{aligned}
$$

Hence

$$
\begin{aligned}
\frac{1}{2}\|p\|_{L^{2}\left(\left(s_{0}, s_{1}\right) \times S^{1}\right)}^{2} & \leq\left\|\eta^{\prime}\right\|_{L^{1}\left(\left(s_{0}, s_{1}\right)\right)}+\left(\|U\|_{\infty}+k\right)\left(s_{1}-s_{0}\right) \\
& \leq \sqrt{s_{1}-s_{0}}\left\|\eta^{\prime}\right\|_{L^{2}\left(\left(s_{0}, s_{1}\right)\right)}+\left(\|U\|_{\infty}+k\right)\left(s_{1}-s_{0}\right) \\
& \leq \sqrt{s_{1}-s_{0}} b_{1}+\left(\|U\|_{\infty}+k\right)\left(s_{1}-s_{0}\right) .
\end{aligned}
$$


Then (4.15) follows with

$$
b_{2}=2 b_{1}+2\left(\|U\|_{\infty}+k\right) .
$$

Next we prove that for any map $u=(x, \eta)$ satisfying the hypotheses of the theorem, and every $0<$ $\varepsilon \leq 1$, the closed subsets

$$
\begin{aligned}
& S_{\varepsilon}(u):=\left\{s \in \mathbb{R}:\|p(s, \cdot)\|_{L^{2}\left(S^{1}\right)}^{2} \leq \frac{b_{2}}{\sqrt{\varepsilon}}\right\} \\
& S_{\varepsilon}^{\prime}(u):=\left\{s \in \mathbb{R}:\left\|x^{\prime}(s, \cdot)\right\|_{L^{2}\left(S^{1}\right)}^{2} \leq \frac{b_{1}}{\sqrt{\varepsilon}}\right\}
\end{aligned}
$$

are $\varepsilon$-dense, that is, they have non-empty intersection with any interval of length $\geq \varepsilon$. Indeed, for every $s_{0} \in \mathbb{R}$ we have that if $0<\varepsilon \leq 1$ then

$$
\begin{aligned}
\min _{s \in\left[s_{0}, s_{0}+\varepsilon\right]}\|p(s, \cdot)\|_{L^{2}\left(S^{1}\right)}^{2} & \leq \frac{1}{\varepsilon} \int_{s_{0}}^{s_{0}+\varepsilon}\|p(s, \cdot)\|_{L^{2}\left(S^{1}\right)}^{2} d s . \\
& =\frac{1}{\varepsilon}\|p\|_{L^{2}\left(\left(s_{0}, s_{0}+\varepsilon\right) \times S^{1}\right)}^{2} \\
& \leq \frac{b_{2}}{\sqrt{\varepsilon}}
\end{aligned}
$$

and hence

$$
S_{\varepsilon}(u) \cap\left[s_{0}, s_{0}+\varepsilon\right] \neq \emptyset .
$$

This proves (4.18). Exactly the same computation with $\left\|x^{\prime}(s, \cdot)\right\|_{L^{2}\left(S^{1}\right)}^{2}$ instead of $\|p(s, \cdot)\|_{L^{2}\left(S^{1}\right)}^{2}$ proves 4.19).

We can now improve (4.15) by finding a constant $b_{3}>0$ such that for all $s \in \mathbb{R}$ it holds that

$$
\|p(s, \cdot)\|_{L^{2}\left(S^{1}\right)} \leq b_{3} .
$$

Indeed, given $s \in \mathbb{R}$, choose $s_{0} \in S_{1}(u)$ such that $\left|s-s_{0}\right| \leq 1$ (i.e. take $\varepsilon=1$ ). Without loss of generality assume $s \geq s_{0}$. Then we have

$$
\begin{aligned}
\|p(s, \cdot)\|_{L^{2}\left(S^{1}\right)}^{2} & =\left\|p\left(s_{0}, \cdot\right)\right\|_{L^{2}\left(S^{1}\right)}^{2}+\int_{s_{0}}^{s} \frac{d}{d r}\|p(r, \cdot)\|_{L^{2}\left(S^{1}\right)}^{2} d r \\
& =\left\|p\left(s_{0}, \cdot\right)\right\|_{L^{2}\left(S^{1}\right)}^{2}+2 \int_{s_{0}}^{s} \int_{S^{1}}\left\langle p(r, t), p^{\prime}(r, t)\right\rangle d t d r \\
& \leq b_{2}+2\left|\int_{s_{0}}^{s}\|p(r, \cdot)\|_{L^{2}\left(S^{1}\right)}^{2} d r\right|^{1 / 2}\left\|p^{\prime}\right\|_{L^{2}\left(\left(s_{0}, s\right) \times S^{1}\right)} \\
& \leq b_{2}+2 \sqrt{b_{2}}\left\|x^{\prime}\right\|_{L^{2}\left(\left(s_{0}, s\right) \times S^{1}\right)} \\
& \leq b_{2}+2 \sqrt{b_{2}} b_{1} .
\end{aligned}
$$

Thus (4.21) follows with

$$
b_{3}:=\sqrt{b_{2}+2 \sqrt{b_{2}} b_{1}}
$$


Next, we show how to improve (4.18) to obtain a similar result with the $L^{2}\left(S^{1}\right)$ norm replaced by the $L^{\infty}\left(S^{1}\right)$ norm. Observe that

$$
\begin{aligned}
\|\dot{p}(s, \cdot)\|_{L^{1}\left(S^{1}\right)} & \leq\|\dot{x}(s, \cdot)\|_{L^{1}\left(S^{1}\right)} \\
& \leq\left\|J(\cdot, x) x^{\prime}(s, \cdot)\right\|_{L^{1}\left(S^{1}\right)}+|\eta(s)|\left\|X_{H}(x(s, \cdot))\right\|_{L^{1}\left(S^{1}\right)} \\
& \leq\|J\|_{\infty}\left\|x^{\prime}(s, \cdot)\right\|_{L^{2}\left(S^{1}\right)}+C_{0} b_{0}\left(1+\|p(s, \cdot)\|_{L^{2}\left(S^{1}\right)}^{2}\right) . \\
& \leq\|J\|_{\infty}\left\|x^{\prime}(s, \cdot)\right\|_{L^{2}\left(S^{1}\right)}+C_{0} b_{0}\left(1+b_{3}^{2}\right),
\end{aligned}
$$

and hence

$$
\begin{aligned}
\|p(s, \cdot)\|_{W^{1,1}\left(S^{1}\right)} & \leq\|p(s, \cdot)\|_{L^{2}\left(S^{1}\right)}+\|\dot{p}(s, \cdot)\|_{L^{1}\left(S^{1}\right)} \\
& \leq b_{3}+\|J\|_{\infty}\left\|x^{\prime}(s, \cdot)\right\|_{L^{2}\left(S^{1}\right)}+C_{0} b_{0}\left(1+b_{3}^{2}\right) .
\end{aligned}
$$

Thus if $N>0$ is the uniform constant such that for any map $f \in W^{1,1}\left(S^{1}, \mathbb{R}\right)$ it holds that

$$
\|f\|_{L^{\infty}\left(S^{1}\right)} \leq N\|f\|_{W^{1,1}\left(S^{1}\right)},
$$

then

$$
\|p(s, \cdot)\|_{L^{\infty}\left(S^{1}\right)} \leq N b_{3}+N\|J\|_{\infty}\left\|x^{\prime}(s, \cdot)\right\|_{L^{2}\left(S^{1}\right)}+N C_{0} b_{0}\left(1+b_{3}^{2}\right) .
$$

Set

$$
b_{4}:=N b_{3}+N C_{0} b_{0}\left(1+b_{3}^{2}\right), \quad b_{5}:=N\|J\|_{\infty} b_{1} .
$$

It now follows from (4.19) and (4.23) that for any $0<\varepsilon \leq 1$ the subset

$$
S_{\varepsilon}^{\prime \prime}(u):=\left\{s \in \mathbb{R}:\|p(s, \cdot)\|_{L^{\infty}\left(S^{1}\right)} \leq b_{4}+\frac{b_{5}}{\sqrt{\varepsilon}}\right\}
$$

has non-empty intersection with any interval of length $\geq \varepsilon$.

Next, we observe that for any $(s, t) \in \mathbb{R} \times S^{1}$, we have

$$
\begin{aligned}
|\nabla p(s, t)|^{2} & \leq|\nabla x(s, t)|^{2} \\
& =\left|x^{\prime}(s, t)\right|^{2}+|\dot{x}(s, t)|^{2} \\
& =\left|x^{\prime}(s, t)\right|^{2}+\left|J(t, x) x^{\prime}(s, t)-\eta(s) X_{H}(x(s, t))\right|^{2} \\
& \stackrel{(*)}{\leq}\left(1+2\|J\|_{\infty}^{2}\right)\left|x^{\prime}(s, t)\right|^{2}+2 b_{0}^{2} C_{0}^{2}\left(1+|p(s, t)|^{2}\right)^{2} \\
& \leq b_{6}\left(1+\left|x^{\prime}(s, t)\right|^{2}+|p(s, t)|^{4}\right)
\end{aligned}
$$

for some constant $b_{6}>0$, where $(*)$ used $|a-b|^{2} \leq 2 a^{2}+2 b^{2}$. Thus for all $s_{0}<s_{1}$ we have

$$
\|\nabla p\|_{L^{2}\left(\left(s_{0}, s_{1}\right) \times S^{1}\right)}^{2} \leq b_{6}\left(\left|s_{1}-s_{0}\right|+b_{1}^{2}\right)+b_{6}\|p\|_{L^{4}\left(\left(s_{0}, s_{1}\right) \times S^{1}\right)}^{4} .
$$

The final step of this part of the proof is to show that there exists $b_{7}>0$ such that for any map $u=(x, \eta)$ satisfying the hypotheses of the theorem, and any finite interval $I \subseteq \mathbb{R}$ we have, writing $x=(q, p)$ that

$$
\|\nabla p\|_{L^{2}\left(I \times S^{1}\right)} \leq b_{7}\left(1+|I|^{1 / 2}\right) .
$$

The proof of (4.26) from (4.25) is based on an interpolation inequality between the $L^{4}$ norm and the $L^{2}$ and $W^{1,2}$ norms, which is due to Abbondandolo and Schwarz. There is no difference between the proof in [3, p278-279] and the one in our situation, so we will omit this. It will be important however in the final section of this paper (see the proof of Proposition 6.2) to state it precisely. The following lemma is not explicitly stated in [3], but follows immediately from a careful inspection of [3, p278-279]. 
4.15. LemmA. Suppose $x=(q, p): \mathbb{R} \rightarrow \Lambda T^{*} M$ is a smooth map such that there exist constants $\gamma_{1}, \gamma_{2}, \gamma_{3}>0$ with the following properties:

(1) $\left\|x^{\prime}\right\|_{L^{2}\left(\mathbb{R} \times S^{1}\right)} \leq \gamma_{1}$;

(2) $\|p(s, \cdot)\|_{L^{2}\left(S^{1}\right)} \leq \gamma_{2}$ for all $s \in \mathbb{R}$;

(3) $\|\nabla p\|_{L^{2}\left(\left(s_{0}, s_{1}\right) \times S^{1}\right)}^{2} \leq \gamma_{3}\left(\left|s_{1}-s_{0}\right|+\gamma_{1}^{2}\right)+\gamma_{3}\|p\|_{L^{4}\left(\left(s_{0}, s_{1}\right) \times S^{1}\right)}^{4}$ for all $s_{0}, s_{1} \in \mathbb{R}$ with $s_{0}<$ $s_{1}$.

Then there exists a constan $70<\varepsilon_{*} \leq 1$ depending only on $\gamma_{2}$ and $\gamma_{3}$ such that if in addition there exists a constant $\gamma_{*}>0$ such that the set

$$
\left\{s \in \mathbb{R}:\|p(s, \cdot)\|_{L^{\infty}\left(S^{1}\right)} \leq \gamma_{*}\right\}
$$

is $\varepsilon_{*}$-dense then there exists a constant $\Gamma=\Gamma\left(\gamma_{1}, \gamma_{2}, \gamma_{3}, \gamma_{*}\right)>0$ such that

$$
\|\nabla p\|_{L^{2}\left(I \times S^{1}\right)} \leq \Gamma\left(1+|I|^{1 / 2}\right)
$$

for any finite interval $I \subseteq \mathbb{R}$.

The important point (as far as Proposition 6.2 is concerned) is that the constant $\Gamma$ depends only on $\gamma_{1}, \gamma_{2}, \gamma_{3}$ and $\gamma_{*}$. Anyway, applying the lemma, (4.26) follows. The proof of Step 1 now follows with $K:=\max \left\{b_{3}, b_{7}\right\}$.

\section{Step 2.}

The next part of the proof shows how the $L^{2}$ estimates (4.13) on $p$ and $\nabla p$ on intervals leads to uniform $L^{\infty}$ bounds. This part of the proof closely follows [3, Theorem 1.14.(i)], and uses the fact that $J \in B_{\varepsilon_{1}}\left(J_{0}\right)$, and the conclusion of Step 1 .

Let $\rho: \mathbb{R} \rightarrow[0,1]$ denote a smooth function such that $\operatorname{supp}(\rho) \subseteq(-1,2),\left.\rho\right|_{[0,1]}=\mathbb{1}$ and $\left|\rho^{\prime}\right| \leq 2$. Given a map $u=(x, \eta)$ satisfying the hypotheses of the theorem and $i \in \mathbb{Z}$, define $x_{i}: \mathbb{R} \rightarrow \Lambda T^{*} M$ by

$$
x_{i}(s, t):=\rho(s-i) x(s, t) .
$$

Note that

$\left(\partial_{s}+J_{0} \partial_{t}\right) x_{i}(s, t)=\rho^{\prime}(s-i) x(s, t)+\rho(s-i) \eta(s) J(t, x) X_{H}(x(s, t))+\rho(s-i)\left(J_{0}(x)-J(t, x)\right) \dot{x}(s, t)$.

Since $x_{i}$ is compactly supported, Theorem 4.13 applies, and we conclude:

$$
\begin{aligned}
\left\|\nabla x_{i}\right\|_{L^{3}\left(\mathbb{R} \times S^{1}\right)} & \leq \frac{1}{2 \varepsilon_{1}}\left\|\left(\partial_{s}+J_{0} \partial_{t}\right) x_{i}\right\|_{L^{3}\left(\mathbb{R} \times S^{1}\right)} \\
& \leq \frac{1}{\varepsilon_{1}}\left(R+\|p\|_{L^{3}\left((i-1, i+2) \times S^{1}\right)}\right)+\frac{C_{0}\|J\|_{\infty}}{2 \varepsilon_{1}}\left\|X_{H}(x)\right\|_{L^{3}\left((i-1, i+2) \times S^{1}\right)} \\
& +\frac{\left\|J_{0}-J\right\|_{\infty}}{2 \varepsilon_{1}}\|\dot{x}\|_{L^{3}\left(\mathbb{R} \times S^{1}\right)},
\end{aligned}
$$

where $R>0$ is a constant depending only on the diameter of the closed manifold $M$.

Given $r>2$, let $P_{r}>0$ denote the constant such that for any $f \in W^{1, r}\left((0,3) \times S^{1}, \mathbb{R}\right)$ it holds that

$$
\|f\|_{L^{r}\left((0,3) \times S^{1}\right)} \leq P_{r}\|f\|_{W^{1,2}\left((0,3) \times S^{1}\right)} .
$$

\footnotetext{
${ }^{7}$ The constant $\varepsilon_{*}$ corresponds to the constant $\delta=1 /\left(32 b_{1} C c_{4}^{2}\right)$ in [3] p279].
} 
Using (4.12) and Step 1 we see that

$$
\begin{aligned}
\left\|X_{H}(x)\right\|_{L^{3}\left((i-1, i+2) \times S^{1}\right)} & \leq b_{0}\left(3^{1 / 3}+\|p\|_{L^{6}\left((i-1, i+2) \times S^{1}\right)}^{2}\right) \\
& \leq b_{0}\left(3^{1 / 3}+P_{6}^{2}\|p\|_{W^{1,2}\left((i-1, i+2) \times S^{1}\right)}^{2}\right) \\
& \leq b_{0}\left(3^{1 / 3}+P_{6}^{2}(K+2 K \sqrt{3})^{2}\right) .
\end{aligned}
$$

Similarly

$$
\|p\|_{L^{3}\left((i-1, i+2) \times S^{1}\right)} \leq P_{3}\|p\|_{W^{1,2}\left((i-1, i+2) \times S^{1}\right)} \leq P_{3}(K+2 K \sqrt{3}) .
$$

Putting this altogether, and using the fact that $J \in B_{\varepsilon_{1}}(J)$ we have therefore proved that there exists a constant $C>0$ that is independent of $u$ and $i$ such that

$$
\left\|\nabla x_{i}\right\|_{L^{3}\left(\mathbb{R} \times S^{1}\right)} \leq C+\frac{1}{2}\|\dot{x}\|_{L^{3}\left(\mathbb{R} \times S^{1}\right)} .
$$

Thus

$$
\|\nabla x\|_{L^{3}\left((i, i+1) \times S^{1}\right)} \leq 2 C .
$$

This gives a uniform bound for $x_{i}$ in $W^{1,3}\left((i, i+1) \times S^{1}\right)$, and hence also in $L^{\infty}\left((i, i+1) \times S^{1}\right)$. Since this bound does not depend on $i$, we have proved the existence of a uniform bound for $x$ in $L^{\infty}\left(\mathbb{R} \times S^{1}\right)$. The theorem follows.

We now turn to the final $L^{\infty}$ estimate we will need. It is based on [3, Theorem 1.14.(iii)]. It will be needed to construct the short exact sequence between the Rabinowitz Floer complex and the Morse (co)complex in the next section. In the statement of the theorem one should substitute either ' + ' or '-' for ' \pm ' throughout.

4.16. THEOREM. There exist constants $\varepsilon_{2}^{ \pm}>0$ with the following property: Suppose $J \in \mathcal{J}(\omega) \cap$ $B_{\varepsilon_{2}^{ \pm}}\left(J_{g}\right)$. Fix $(g, \sigma, U, k) \in \mathcal{O}, R>0, \alpha \in\left[S^{1}, M\right]$ and $-\infty<a<b<\infty$. Let $H:=H_{g}+\pi^{*} U$. Then there exist constants $C_{4}^{ \pm}, C_{5}^{ \pm}>0$ such that for any map

$$
u=(x, \eta): \mathbb{R}^{ \pm} \times S^{1} \rightarrow T^{*} M \times \mathbb{R}
$$

with

$$
\begin{gathered}
x \in C^{\infty}\left(\mathbb{R}^{ \pm} \times S^{1}, T^{*} M\right) \cap W^{1,3}\left((0, \pm 1) \times S^{1}, T^{*} M\right) ; \\
\eta \in C^{\infty}\left(\mathbb{R}^{ \pm}, \mathbb{R}\right) \cap W^{1,3}((0, \pm 1), \mathbb{R}),
\end{gathered}
$$

that satisfies the Rabinowitz Floer equation on $\mathbb{R}^{ \pm} \times S^{1}$, has action bounds $A_{H-k}\left(u\left(\mathbb{R}^{ \pm}\right)\right) \subseteq[a, b]$ together with the extra assumptions:

$$
\begin{gathered}
\pm \eta(0) \geq 0 ; \\
\|q(0, \pm \cdot)\|_{W^{2 / 3,3}\left(S^{1}, \mathbb{R}^{d}\right)} \leq R
\end{gathered}
$$

(where here $d$ is such that $(M, g)$ embeds isometrically into $\left(\mathbb{R}^{d}, g_{0}\right)$, and we have written $x=(q, p)$ ), it holds that:

$$
\|\eta\|_{L^{\infty}\left(\mathbb{R}^{ \pm}\right)} \leq C_{4}^{ \pm}, \quad\|x\|_{L^{\infty}\left(\mathbb{R}^{ \pm} \times S^{1}\right)} \leq C_{5}^{ \pm}
$$

Proof. Firstly, the proof of Theorem 4.11 will still go through for flow lines defined on $\mathbb{R}^{+}$instead of $\overline{\mathbb{R}}$, provided we have an a priori lower bound on $\eta(0)$. If $u$ is defined on $\mathbb{R}^{-}$then the proof will go through provided we (a) have an a priori upper bound on $\eta(0)$, and (b), we we redefine the function $\tau(s)$ from (4.11) to be

$$
\tau(s):=\inf \left\{r \geq 0:\left\|\nabla A_{H-k}(u(s-r, \cdot))\right\|_{J} \leq \rho_{0}\right\} .
$$

Therefore we have proved the existence of constants $C_{4}^{ \pm}>0$ that uniformly bound the $\eta$-component of any map $u$ satisfying the hypotheses of the theorem. Now Step 1 from the proof of Theorem 4.14 goes through without any essential changes (save of course from the fact that now $u$ is defined on $\mathbb{R}^{ \pm}$). 
The proof of Step 2 also proceeds similarly, aside from the fact that instead of following [3, Theorem 1.14.(ii)] we must instead follow [3, Theorem 1.14.(iii)]. In particular, the constants $\varepsilon_{2}^{ \pm}>0$ in the statement of the theorem come from a version of Theorem 4.13 for maps defined on $\mathbb{R}^{ \pm} \times S^{1}$ instead of $\mathbb{R} \times S^{1}$.

\section{THE ABbONDANDOLO-SCHWARZ SHORT EXACT SEQUENCE}

In this section we state and prove the main result of the paper, which is the extension of [4, Theorem 2] to the weakly exact case. In the statement of the theorem below it is implicitly assumed that $\|\sigma\|_{\infty}$ is sufficiently small; this ensures that almost complex structures that fit the hypotheses of theorem exist, cf. Remark 4.9

5.1. Theorem. Fix $(g, \sigma, U, k) \in \mathcal{O}_{\text {reg }}$ and $\alpha \in\left[S^{1}, M\right]$. Put $H=H_{g}+\pi^{*} U$ and $L=L_{g}-$ $\pi^{*} U$. Let $f$ and $h$ be Morse functions on $\overline{\operatorname{Crit}}\left(S_{L+k}\right)$ and $\operatorname{Crit}\left(A_{H-k}\right)$ satisfying certain compatibility requirements (stated precisely in Subsection 5.1 below). Let $J \in \mathcal{J}(\omega)$ denote a generically chosen almost complex structure lying sufficiently close to the metric almost complex structure $J_{g}$. Let $G$ denote a generically chosen metric on $\Lambda M \times \mathbb{R}^{+}$that is uniformly equivalent to $\langle\langle\cdot, \cdot\rangle\rangle_{g}$, and let $g_{0}$ and $g_{1}$ denote generically chosen Riemannian metrics on $\overline{\operatorname{Crit}}\left(S_{L+k}\right)$ and $\operatorname{Crit}\left(A_{H-k}\right)$ respectively, such that the negative gradient flows of $f$ and $h$ with respect to these metrics are Morse-Smale. Then there exists:

(1) An injective chain map $\Phi_{\mathrm{SA}}: C M_{*}\left(S_{L+k}, f ; \alpha\right) \rightarrow R F_{*}\left(A_{H-k}, h ; \alpha\right)$ which admits a left inverse $\widehat{\Phi}_{\mathrm{SA}}: R F_{*}\left(A_{H-k}, h ; \alpha\right) \rightarrow C M_{*}\left(S_{L+k}, f ; \alpha\right)$.

(2) A surjective chain map $\Phi_{\mathrm{AS}}: R F_{*}\left(A_{H-k}, h ; \alpha\right) \rightarrow C M^{1-*}\left(S_{L+k},-f ;-\alpha\right)$ which admits a right inverse $\widehat{\Phi}_{\mathrm{AS}}: C M^{1-*}\left(S_{L+k},-f ;-\alpha\right) \rightarrow R F_{*}\left(A_{H-k}, h ; \alpha\right)$.

Moreover the composition $\Phi_{\mathrm{AS}} \circ \Phi_{\mathrm{SA}}: C M_{*}\left(S_{L+k}, f ; \alpha\right) \rightarrow C M^{1-*}\left(S_{L+k},-f ;-\alpha\right)$ is chain homotopic to zero, that is, there exists a homomorphism $P: C M_{*}\left(S_{L+k}, f ; \alpha\right) \rightarrow C M^{-*}\left(S_{L+k},-f ;-\alpha\right)$ such that

$$
\Phi_{\mathrm{AS}} \circ \Phi_{\mathrm{SA}}=P \partial^{\text {Morse }}+\delta^{\text {Morse }} P .
$$

Setting

$$
\Theta:=\Phi_{\mathrm{SA}}-\widehat{\Phi}_{\mathrm{AS}} P \partial^{\text {Morse }}-\partial^{\text {Morse }} \widehat{\Phi}_{\mathrm{AS}} P,
$$

the chain map $\Theta$ is chain homotopic to $\Phi_{\mathrm{SA}}$, and satisfies $\Phi_{\mathrm{AS}} \circ \Theta=0$, and thus we obtain a short exact sequence of chain complexes

$$
0 \rightarrow C M_{*}\left(S_{L+k}, f ; \alpha\right) \stackrel{\Theta}{\rightarrow} R F_{*}\left(A_{H-k}, h ; \alpha\right) \stackrel{\Phi_{\mathrm{AS}}}{\rightarrow} C M^{1-*}\left(S_{L+k},-f ;-\alpha\right) \rightarrow 0 .
$$

Identifying $H M_{*}\left(S_{L+k}, f ; \alpha\right) \cong H_{*}\left(\Lambda_{\alpha} M ; \mathbb{Z}_{2}\right)$ and $H M^{*}\left(S_{L+k},-f ;-\alpha\right) \cong H^{*}\left(\Lambda_{-\alpha} M ; \mathbb{Z}_{2}\right)$, and passing to the associated long exact sequence

$$
\cdots \longrightarrow H_{i}\left(\Lambda_{\alpha} M ; \mathbb{Z}_{2}\right) \stackrel{\Theta_{*}}{\longrightarrow} R F H_{i}\left(A_{H-k} ; \alpha\right) \stackrel{\left(\Psi_{\mathrm{AS}}\right)_{*}}{\longrightarrow} H^{1-i}\left(\Lambda_{-\alpha} M ; \mathbb{Z}_{2}\right) \stackrel{\Delta}{\longrightarrow} H_{i-1}\left(\Lambda_{\alpha} M ; \mathbb{Z}_{2}\right) \longrightarrow \cdots
$$

the connecting homomorphism $\Delta$ is identically zero unless $\alpha=0$ and $i=0$, in which case it is multiplication by the Euler class $e\left(T^{*} M\right)$. This therefore allows one to obtain a complete description of the Rabinowitz Floer homology of $A_{H-k}$.

As mentioned in the introduction, the proof of this theorem is now essentially identical to the corresponding proof in [4]. We therefore omit almost all of the technical details, referring the reader to the beautiful and lucid exposition in [4], and instead just give an outline of Abbondandolo and Schwarz' constructions. 


\subsection{Choosing the Morse functions $f$ and $h$.}

In order to construct the chain homotopy $P$ in the theorem above it is essential that the Morse functions $f: \overline{\operatorname{Crit}}\left(S_{L+k}\right) \rightarrow \mathbb{R}$ and $h: \operatorname{Crit}\left(A_{H-k}\right) \rightarrow \mathbb{R}$ are chosen in such a way that certain compatibility requirements are satisfied. More precisely, we require that the following four conditions are satisfied:

(1) For all $w \in \operatorname{Crit}(f)$, it holds that $f(w)=h\left(Z^{ \pm}(w)\right)$, and $i_{f}(w)=i_{h}\left(Z^{ \pm}(w)\right)$.

(2) The function $\left.f\right|_{M \times\{0\}}$ has a unique minimum and a unique maximum and is self-indexing, that is, $f(q, 0)=i_{f}((q, 0))$ for all $(q, 0) \in \overline{\operatorname{Crit}}(f) \backslash \operatorname{Crit}(f)$.

(3) For all $x \in \Sigma_{k}$, we have $f(\pi(x), 0) \leq h(x, 0) \leq f(\pi(x), 0)+1 / 2$.

(4) Every critical point of $\left.h\right|_{\Sigma_{k} \times\{0\}}$ lies above a critical point of $\left.f\right|_{M \times\{0\}}$, and moreover for each critical point $(q, 0)$ of $\left.f\right|_{M \times\{0\}}$ there are exactly two critical points of $\left.h\right|_{\Sigma_{k} \times\{0\}}$ in the fibre $\left(\Sigma_{k} \cap T_{q}^{*} M\right) \times\{0\}$. Denoting these two critical points by $\left(x_{q}^{ \pm}, 0\right)$, it holds that $f(q, 0)=$ $h\left(x_{q}^{-}, 0\right)=h\left(x_{q}^{+}, 0\right)-1 / 2$, and that $i_{f}(q, 0)=i_{h}\left(x_{q}^{-}, 0\right)=i_{h}\left(x_{q}^{+}, 0\right)-n+1$.

Such functions exist because $k>e_{0}(g, \sigma, U)$. This is explained in detail in [4, Appendix B]. An immediate consequence of these requirements and Proposition 4.6 is the following result.

5.2. Lemma. Assume that the Morse functions $f: \overline{\operatorname{Crit}}\left(S_{L+k}\right) \rightarrow \mathbb{R}$ and $h: \operatorname{Crit}\left(A_{H-k}\right) \rightarrow \mathbb{R}$ satisfy the requirements above.

Then

$$
\begin{gathered}
\widehat{i}_{f}(w)=\widehat{\mu}_{h}\left(Z^{+}(w)\right) \\
\widehat{i}_{-f}(w)=1-\widehat{\mu}_{h}\left(Z^{-}(w)\right)
\end{gathered}
$$

for $w \in \operatorname{Crit}(f)$ and

$$
\begin{gathered}
\widehat{i}_{f}((q, 0))=n-\widehat{\mu}_{h}\left(x_{q}^{+}, 0\right) \\
\widehat{i}_{-f}((q, 0))=1-\widehat{\mu}_{h}\left(x_{q}^{-}, 0\right)
\end{gathered}
$$

for $(q, 0) \in \overline{\operatorname{Crit}}(f) \backslash \operatorname{Crit}(f)$.

\subsection{The chain map $\Phi_{\mathrm{SA}}$.}

In order to define the chain map $\Phi_{\mathrm{SA}}$, one first needs to construct a suitable moduli space. Here are the details. Recall that $G$ denotes a metric on $\Lambda M \times \mathbb{R}^{+}$that is uniformly equivalent to $\langle\langle\cdot, \cdot\rangle\rangle_{g}$ and $g_{0}$ is a Riemannian metric on $\overline{\operatorname{Crit}}\left(S_{L+k}\right)$ such that the negative gradient flow $\phi_{t}^{-\nabla f}$ of $-\nabla f$ is Morse-Smale, and $g_{1}$ is a Riemannian metric on $\overline{\operatorname{Crit}}\left(A_{H-k}\right)$ such that the negative gradient flow $\phi_{t}^{-\nabla h}$ is Morse-Smale. Fix a generic almost complex structure $J \in \mathcal{J}(\omega) \cap B_{\varepsilon_{2}^{+}}\left(J_{g}\right)$ (where $\varepsilon_{2}^{+}>0$ is the constant from Theorem 4.16).

Fix $w \in \overline{\operatorname{Crit}}(f)$. If $m \in \mathbb{N}$, let $\widetilde{\mathcal{W}}_{m}^{-}(w)$ denote the set of tuples $\boldsymbol{w}=\left(w_{1}, \ldots, w_{m}\right)$ such that $w_{i} \in \Lambda M \times \mathbb{R}^{+}$for $i=1, \ldots, m-1$ and $w_{m} \in \Lambda M \times \mathbb{R}_{0}^{+}$, and such that

$$
\begin{gathered}
w_{1} \in W^{u}\left(W^{u}(w ;-\nabla f) ;-\nabla S_{L+k}\right) ; \\
\Psi_{-\infty}\left(w_{i+1}\right) \in \phi_{\mathbb{R}^{+}}^{-\nabla f}\left(\Psi_{\infty}\left(w_{i}\right)\right) .
\end{gathered}
$$

Let $\mathcal{W}_{m}^{-}(w)$ denote the quotient of $\widetilde{\mathcal{W}}_{m}^{-}(w)$ under the free $\mathbb{R}^{m-1}$ action given by

$$
\left(w_{1}, \ldots, w_{m-1}\right) \mapsto\left(\Psi_{s_{1}}\left(w_{1}\right), \ldots, \Psi_{s_{m-1}}\left(w_{m-1}\right)\right), \quad\left(s_{1}, \ldots, s_{m-1}\right) \in \mathbb{R}^{m-1} .
$$

Then put

$$
\mathcal{W}^{-}(w):=\bigcup_{m \in \mathbb{N} \cup\{0\}} \mathcal{W}_{m}^{-}(w)
$$


For generically chosen $G$ and $g_{0}, \mathcal{W}^{-}(w)$ has the structure of a smooth finite dimensional manifold of dimension $\widehat{i}_{f}(w)$.

Fix $z \in \operatorname{Crit}(h)$. Let $\widetilde{\mathcal{M}}_{m}^{+}(z)$ denote the denote the set of tuples of maps $\boldsymbol{u}=\left(u_{1}, \ldots, u_{m}\right)$ such that

$$
\begin{gathered}
u_{1}: \mathbb{R}_{0}^{+} \rightarrow C^{\infty}\left(S^{1}, T^{*} M\right) \times \mathbb{R} ; \\
u_{2}, \ldots, u_{m}: \mathbb{R} \rightarrow C^{\infty}\left(S^{1}, T^{*} M\right) \times \mathbb{R},
\end{gathered}
$$

all satisfy the Rabinowitz Floer equation (4.3) (which are possibly stationary solutions) and such that

$$
\begin{gathered}
u_{m}(\infty) \in W^{s}(z ;-\nabla h) ; \\
u_{i+1}(-\infty) \in \phi_{\mathbb{R}^{+}}^{-\nabla h}\left(u_{i}(\infty)\right) \text { for } i=1, \ldots, m-1 .
\end{gathered}
$$

Let $\mathcal{M}_{m}^{+}(z)$ denote the quotient of $\widetilde{\mathcal{M}}_{m}^{+}(z)$ under the free $\mathbb{R}^{m-1}$ action given by translation along the flow lines $u_{2}, \ldots, u_{m}$.

Then put

$$
\mathcal{M}^{+}(z):=\bigcup_{m \in \mathbb{N} \cup\{0\}} \mathcal{M}_{m}^{+}(z) .
$$

The space $\mathcal{M}^{+}(z)$ is not finite dimensional. However, by restricting where the tuple $\boldsymbol{u}$ can "begin", we can cut it down to something finite dimensional. This is precisely what the moduli space $\mathcal{M}_{\mathrm{SA}}(w, z)$ does. Namely, the moduli space $\mathcal{M}_{\mathrm{SA}}(w, z)$ is defined to be the following subset of $\mathcal{W}^{-}(w) \times \mathcal{M}^{+}(z)$. A pair $([\boldsymbol{w}],[\boldsymbol{u}]$ ) (where the square brackets denote the equivalence class after dividing through by the translation actions) belongs to $\mathcal{M}_{\mathrm{SA}}(w, z)$ if and only if we have, writing

$$
\begin{gathered}
\boldsymbol{w}=\left(w_{1}, \ldots, w_{m}\right) ; \\
\boldsymbol{u}=\left(u_{1}, \ldots, u_{j}\right) \quad \text { with } u_{j}=\left(x_{j}, \eta_{j}\right),
\end{gathered}
$$

that

$$
w_{m}=\left(\pi \circ x_{1}(0), \eta_{1}(0)\right) \text {. }
$$

In other words, the tuple $\boldsymbol{w}$ must "end" where the tuple $\boldsymbol{u}$ "begins".

For a fixed element $w^{*} \in \Lambda M \times \mathbb{R}_{0}^{+}$, requiring tuples $\boldsymbol{u}$ to "begin" at $w^{*}$ in the sense that $(\pi \circ$ $\left.x_{1}(0), \eta_{1}(0)\right)=w^{*}$ defines a Lagrangian boundary condition. This implies that we have a Fredholm problem, and since generically $\mathcal{W}^{-}(w)$ is a finite dimensional manifold, it follows that $\mathcal{M}_{\mathrm{SA}}(w, z)$ can be seen as the zero set of a Fredholm operator, whose index can be computed to be $\widehat{i}_{f}(w)-\widehat{\mu}_{h}(z)$. In fact, more is true. Namely, $\mathcal{M}_{\mathrm{SA}}(w, z)$ (for generic $G, g_{0}, J$ and $\left.g_{1}\right)$ is a precompact finite dimensional manifold of dimension $\widehat{i}_{f}(w)-\widehat{\mu}_{h}(z)$.

This requires us to check two more things. Firstly, one needs to have $C_{\mathrm{loc}}^{\infty}$-bounds for the curves $\boldsymbol{u}=\left(u_{1}, \ldots, u_{j}\right)$. Here the following key inequality comes into play. Given $([\boldsymbol{w}],[\boldsymbol{u}]) \in \mathcal{M}_{\mathrm{SA}}(w, z)$, equation (4.1) from Lemma4.1 tells us that for all $s \in \mathbb{R}^{+}$:

$$
\begin{aligned}
S_{L+k}(w) \geq S_{L+k}\left(w_{i}\right) \geq S_{L+k}\left(w_{m}\right)=S_{L+k}( & \left.\pi \circ x_{1}(0, \cdot), \eta_{1}(0)\right) \\
& \geq A_{H-k}\left(u_{1}(0, \cdot)\right) \geq A_{H-k}\left(u_{i}(s, \cdot)\right) \geq A_{H-k}(z) .
\end{aligned}
$$

Then uniform $L^{\infty}$ estimates for the solutions $u_{2}, \ldots, u_{j}$ come from Theorem 4.14, and the uniform $L^{\infty}$ estimate for $u_{1}$ comes from Theorem 4.16. As before, these $L^{\infty}$ bounds give us $C_{\text {loc }}^{\infty}$ bounds (since $\left.\omega\right|_{\pi_{2}(M)}=0$ and $\left.c_{1}\left(T^{*} M, \omega\right)=0\right)$. This shows that the moduli spaces $\mathcal{M}_{\mathrm{SA}}(w, z)$ are compact up to breaking.

The only complication with obtaining transversality is the presence of stationary solutions, which can appear if $z=Z^{+}(w)$ or $w=(q, 0) \in \overline{\operatorname{Crit}}(f) \backslash \operatorname{Crit}(f)$ is a critical point at infinity and $z=\left(x_{q}^{ \pm}, 0\right)$ is one of the corresponding two critical points of $h$. In the former case the first inequality of the third statement of Lemma 4.1 forces the linearized operator defining the moduli space $\mathcal{M}_{\mathrm{SA}}\left(w, Z^{+}(w)\right)$ to 
be an isomorphism (see [4, Lemma 6.2] or [3, Proposition 3.7]), and in the second two cases the four assumptions made earlier on the Morse functions $f$ and $a$ guarantee that the linearized operator defining the moduli spaces $\mathcal{M}_{\mathrm{SA}}\left((q, 0),\left(x_{q}^{ \pm}, 0\right)\right)$ is surjective (see [4, Lemma 6.3]).

Putting this together, we deduce that when $\widehat{i}_{f}(w)=\widehat{\mu}_{h}(z)$, the moduli space $\mathcal{M}_{\mathrm{SA}}(w, z)$ is a finite set, and hence we can define

$$
n_{\mathrm{SA}}(w, z):=\# \mathcal{M}_{\mathrm{SA}}(w, z) \text { taken modulo } 2 .
$$

Then one defines $\Phi_{\mathrm{SA}}: C M_{*}\left(S_{L+k}, f\right) \rightarrow R F_{*}\left(A_{H-k}, h\right)$ by

$$
\Phi_{\mathrm{SA}} w=\sum_{z \in \operatorname{Crit}_{i}(h)} n_{\mathrm{SA}}(w, z) z, \quad w \in{\overline{\operatorname{Crit}_{i}}}_{i}(f) .
$$

A standard gluing argument shows that $\Phi_{\mathrm{SA}}$ is a chain map. It is clear that $\Phi_{\mathrm{SA}}$ restricts to define a chain map $C M\left(S_{L+k}, f ; \alpha\right) \rightarrow R F_{*}\left(A_{H-k}, h ; \alpha\right)$ for each $\alpha \in\left[S^{1}, M\right]$.

\subsection{The chain map $\Phi_{\mathrm{AS}}$.}

The chain map $\Phi_{\mathrm{AS}}$ is defined in much the same way. One begins by defining spaces $\mathcal{M}^{-}(z)$ for $z \in \operatorname{Crit}(h)$. Let $\widetilde{\mathcal{M}}_{m}^{-}(z)$ denote the denote the set of tuples of maps $\boldsymbol{u}=\left(u_{1}, \ldots, u_{m}\right)$ such that

$$
\begin{gathered}
u_{1}, \ldots, u_{m-1}: \mathbb{R} \rightarrow C^{\infty}\left(S^{1}, T^{*} M\right) \times \mathbb{R} ; \\
u_{m}: \mathbb{R}_{0}^{-} \rightarrow C^{\infty}\left(S^{1}, T^{*} M\right) \times \mathbb{R},
\end{gathered}
$$

all satisfy the Rabinowitz Floer equation 4.3 (which are possibly stationary solutions) and such that

$$
u_{-}(\infty) \in W^{u}(z ;-\nabla h),
$$

and such that

$$
u_{i+1}(-\infty) \in \phi_{\mathbb{R}^{+}}^{-\nabla h}\left(u_{i}(\infty)\right) \text { for } i=1, \ldots, m-1 .
$$

Let $\mathcal{M}_{m}^{-}(z)$ denote the quotient of $\widetilde{\mathcal{M}}_{m}^{-}(z)$ under the free $\mathbb{R}^{m-1}$ action and put

$$
\mathcal{M}^{-}(z):=\bigcup_{m \in \mathbb{N}} \mathcal{M}_{m}^{-}(z)
$$

Given $z \in \operatorname{Crit}(h)$ and $w \in \overline{\operatorname{Crit}}(-f)$, the moduli space $\mathcal{M}_{\mathrm{AS}}(z, w)$ consists of the subset $\mathcal{M}^{-}(z) \times$ $\mathcal{W}^{-}(w)$ of elements $([\boldsymbol{u}],[\boldsymbol{w}])$ such that, writing

we have

$$
\begin{gathered}
\boldsymbol{u}=\left(u_{1}, \ldots, u_{j}\right) \quad \text { with } u_{i}=\left(x_{i}, \eta_{i}\right) ; \\
\boldsymbol{w}=\left(w_{1}, \ldots, w_{m}\right) \quad \text { with } w_{i}=\left(q_{i}, T_{i}\right),
\end{gathered}
$$

$$
\left(q_{m}(t), T_{m}\right)=\left(\pi \circ x_{j}(0,-t),-\eta_{j}(0)\right) .
$$

This time the moduli space $\mathcal{M}_{\mathrm{AS}}(z, w)$ admits the structure of a precompact smooth manifold of finite dimension $\widehat{\mu}_{h}(z)+\widehat{i}_{-f}(w)-1$. Here one uses equation (4.2) from Lemma4.1 to deduce the inequality

$$
\begin{aligned}
& A_{H-k}(z) \geq A_{H-k}\left(u_{i}(s, \cdot)\right) \geq A_{H-k}\left(u_{j}(0, \cdot)\right) \\
& \quad \geq-S_{L+k}\left(\pi \circ x_{j}(0,-\cdot),-\eta_{j}(0)\right) \geq-S_{L+k}\left(w_{m}\right) \geq-S_{L+k}\left(w_{i}\right) \geq-S_{L+k}(w),
\end{aligned}
$$

which gives the required $L^{\infty}$ estimates on the $u_{i}$, and the second inequality in the third statement of Lemma 4.1 to obtain the automatic transversality in the case $z=Z^{-}(w)$. Thus if $z \in \operatorname{Crit}(h)$ and $w \in \overline{\operatorname{Crit}}(-f)$ satisfy $\widehat{\mu}_{h}(z)+\widehat{i}_{-f}(w)=1, \mathcal{M}_{\mathrm{AS}}(z, w)$ is a finite set, and hence we may define $n_{\mathrm{AS}}(z, w)$ to be its parity. This defines the chain map $\Phi_{\mathrm{AS}}$. As before $\Phi_{\mathrm{AS}}$ restricts to define a chain map $R F_{*}\left(A_{H-k}, h ; \alpha\right) \rightarrow C M^{1-*}\left(S_{L+k}, f ;-\alpha\right)$ for each $\alpha \in\left[S^{1}, M\right]$. 


\subsection{The chain homotopy $P$.}

The final ingredient is the chain homotopy $P: C M_{*}\left(S_{L+k}, f\right) \rightarrow C M^{-*}\left(S_{L+k},-f\right)$. This involves counting a slightly different sort of object. Let $\mathcal{F}_{0}$ denote the set of pairs $(u, T)$ where $T \in \mathbb{R}^{+}$and $u:[-T, T] \rightarrow T^{*} M \times \mathbb{R}$ satisfies the Rabinowitz Floer equation (4.3). Given $m \geq 1$, let $\widetilde{\mathcal{F}}_{m}$ denote the set of tuples $\boldsymbol{u}=\left(u_{0}, \ldots, u_{m}\right)$ such that

$$
\begin{gathered}
u_{0}: \mathbb{R}^{+} \rightarrow C^{\infty}\left(S^{1}, T^{*} M\right) \times \mathbb{R} ; \\
u_{2}, \ldots, u_{m-1}: \mathbb{R} \rightarrow C^{\infty}\left(S^{1}, T^{*} M\right) \times \mathbb{R} ; \\
u_{m}: \mathbb{R}^{-} \rightarrow C^{\infty}\left(S^{1}, T^{*} M\right) \times \mathbb{R},
\end{gathered}
$$

all satisfy the Rabinowitz Floer equation (4.3), and such that

$$
u_{i}(-\infty) \in \phi_{\mathbb{R}^{+}}^{-\nabla h}\left(u_{i-1}(-\infty)\right) \text { for } i=1, \ldots, m .
$$

Let $\mathcal{F}_{m}$ denote the quotient of $\widetilde{\mathcal{F}}_{m}$ by dividing through by the $\mathbb{R}^{m-1}$ action on the middle curves $u_{1}, \ldots, u_{m-1}$. Put

$$
\mathcal{F}=\bigcup_{m \in \mathbb{N} \cup\{0\}} \mathcal{F}_{m}
$$

Given $w_{-}, w_{+} \in \overline{\operatorname{Crit}}(f)$, denote by $\mathcal{M}_{P}\left(w_{-}, w_{+}\right)$the subset of $\left.\mathcal{W}^{-}\left(w_{-}\right) \times \mathcal{F} \times \mathcal{W}^{-} w_{+}\right)$of elements that "begin" at $w^{-}$and "pass through" an element of $\mathcal{F}$ and then "end" at $w^{+}$(we refer to [4, p46-47] for the precise definition). Then $\mathcal{M}_{P}\left(w_{-}, w_{+}\right)$turns out to be a finite dimensional smooth manifold of dimension $\widehat{i}_{f}\left(w_{-}\right)+\widehat{i}_{f}\left(w_{+}\right)$. Here the key issue in the analysis is to check that if $(u, T) \in \mathcal{F}_{0}$ then $T$ is strictly bounded away from zero ([4, Lemma 8.2]).

Now we move onto the key proposition behind the proof of Theorem 5.1. The first statement belows shows that if $w_{ \pm} \in \overline{\operatorname{Crit}}(\mp f)$ satisfy $\widehat{i}_{f}\left(w_{-}\right)+\widehat{i}_{-f}\left(w_{+}\right)=1$, we can define $n_{P}\left(w_{-}, w_{+}\right)$as the parity of the finite set $\mathcal{M}_{P}\left(w_{-}, w_{+}\right)$. This defines the map $P$. The fact that $P$ is a chain homotopy between $\Phi_{\mathrm{SA}}$ and $\Phi_{\mathrm{AS}}$ involves studying the compactification of $\mathcal{M}_{P}\left(w_{-}, w_{+}\right)$by adding in the broken trajectories, and is the content of the second and third statements of the proposition below.

5.3. Proposition. ([4, Proposition 8.1])

Let $\alpha \in\left[S^{1}, M\right]$ and choose

$$
\begin{gathered}
w_{0} \in \overline{\operatorname{Crit}}_{0}(f ; \alpha), \quad w_{1} \in \overline{\operatorname{Crit}}_{1}(f ; \alpha) ; \\
w^{0} \in \overline{\operatorname{Crit}}_{0}(-f ;-\alpha), \quad w^{1} \in \overline{\operatorname{Crit}}_{1}(-f ;-\alpha) .
\end{gathered}
$$

Then:

(1) The moduli space $\mathcal{M}_{P}\left(w_{0}, w^{0}\right)$ is compact.

(2) The moduli space $\mathcal{M}_{P}\left(w_{0}, w^{1}\right)$ is precompact, and we can identify the boundary $\partial \widehat{\mathcal{M}}_{P}\left(w_{0}, w^{1}\right)$ of compactification $\widehat{\mathcal{M}}_{P}\left(w_{0}, w^{1}\right)$ as follows:

$$
\begin{aligned}
\partial \widehat{\mathcal{M}}_{P}\left(w_{0}, w^{1}\right)= & \left\{\bigcup_{z \in \operatorname{Crit}_{0}(h) \cap \operatorname{Crit}\left(A_{H-k} ; \alpha\right)} \mathcal{M}_{\mathrm{SA}}\left(w_{0}, z\right) \times \mathcal{M}_{\mathrm{AS}}\left(z, w^{1}\right)\right\} \\
& \bigcup\left\{\bigcup_{w \in \overline{\operatorname{Crit}}_{0}(-f) \cap \operatorname{Crit}\left(S_{L+k} ;-\alpha\right)} \mathcal{M}_{P}\left(w_{0}, w\right) \times \mathcal{W}\left(w, w^{1}\right)\right\} .
\end{aligned}
$$


(3) The moduli space $\mathcal{M}_{P}\left(w_{1}, w^{0}\right)$ is precompact, and we can identify the boundary $\partial \widehat{\mathcal{M}}_{P}\left(w_{1}, w^{0}\right)$ of compactification $\widehat{\mathcal{M}}_{P}\left(w_{1}, w^{0}\right)$ as follows:

$$
\begin{aligned}
\partial \widehat{\mathcal{M}}_{P}\left(w_{1}, w^{0}\right)= & \left\{\bigcup_{z \in \operatorname{Crit}_{1}(h) \cap \operatorname{Crit}\left(A_{H-k} ; \alpha\right)} \mathcal{M}_{\mathrm{SA}}\left(w_{1}, z\right) \times \mathcal{M}_{\mathrm{AS}}\left(z, w^{0}\right)\right\} \\
& \bigcup\left\{\bigcup_{w \in \overline{\operatorname{Crit}}_{1}(-f) \cap \operatorname{Crit}\left(S_{L+k} ;-\alpha\right)} \mathcal{W}\left(w_{1}, w\right) \times \mathcal{M}_{P}\left(w, w^{0}\right)\right\} .
\end{aligned}
$$

Theorem 5.1 essentially follows from this proposition; see [4, Section 9] for the details.

\section{NON-DISPLACEABILITY AND LEAF-WISE INTERSECTIONS ABOVE THE CRITICAL VALUE}

\subsection{Relating $R F H_{*}\left(A_{H-k}\right)$ with $R F H_{*}\left(\Sigma_{k}, T^{*} M\right)$.}

Rabinowitz Floer homology was defined originally in [16] for restricted contact type hypersurfaces and Hamiltonians which are constant at infinity. This was extended in [20] to cover (amongst other things) the hypersurfaces $\Sigma_{k}$ that we study here. A natural question therefore becomes whether the Rabinowitz Floer homology we work with in this paper is isomorphic to that of [20]. The aim of this section is to prove this in the affirmative.

Let $(g, \sigma, U, k) \in \mathcal{O}_{\text {reg }}$ and put $H=H_{g}+\pi^{*} U$ and $\Sigma_{k}:=H^{-1}(k)$. Let $\rho: \mathbb{R} \rightarrow(-\infty, 1]$ denote a smooth function

$$
\rho(t):=\left\{\begin{array}{ll}
t & t \in(-\infty, 1-\delta] \\
1 & t \in[1+\delta, \infty)
\end{array} \quad 0 \leq \rho^{\prime} \leq 1,\right.
$$

where $0<\delta<1 / 3$. Given $R>1$, let $\rho_{R}(t):=R \rho\left(\frac{t}{R}\right)$. Let $H_{R}: T^{*} M \rightarrow \mathbb{R}$ be defined by

$$
H_{R}:=\rho_{R} \circ H \text {. }
$$

Assuming $R \gg k$, the Hamiltonian $H_{R}$ satisfies $\Sigma_{k}=H_{R}^{-1}(k)$ and $\left.X_{H_{R}}\right|_{\Sigma_{k}}=\left.X_{H}\right|_{\Sigma_{k}}$. However the Hamiltonian $H_{R}$ is constant at infinity. This makes no difference to the proof of Theorem 4.11, or to that of Step 2 in the proof of Theorem 4.14, but the proof of Step 1 of Theorem 4.14 explicitly required the Hamiltonian to be quadratic. In the course of the proof below we will show that the proof of Theorem 4.14 will still go though for the Hamiltonian $H_{R}$, provided $R \gg 0$ is sufficiently large. However exactly what constitutes "sufficiently large" depends on the action interval $(a, b) \subseteq \mathbb{R}$. Thus this method is not good enough to define the full Rabinowitz Floer homology with $H_{R}$.

6.1. REMARK. In [20] this is overcome by using an entirely different method to obtain $L^{\infty}$ bounds on the $x$-component of gradient flow lines. Namely, they work with a compatible almost complex structure $J$ that is geometrically bounded outside of a compact set. We refer to [11, Chapter V] for the precise definition, and also for an explanation as to why working with Hamiltonians which are constant outside of a compact set and almost complex structures that are geometrically bounded outside of a compact set leads to such $L^{\infty}$ bounds. Proofs that twisted cotangent bundles are geometrically bounded can be found in [21, Proposition 2.2] or [34, Proposition 4.1]. The latter proof also shows that it is possible (if $\|\sigma\|_{\infty}$ is small enough) to find geometrically bounded almost complex structures $J \in \mathcal{J}(\omega) \cap B_{\varepsilon_{1}}\left(J_{g}\right)$.

We now prove the following result.

6.2. Proposition. Given a fixed finite interval $(a, b) \subseteq \mathbb{R}$, there exists a constant $R(a, b)>0$ such that for all $R>R(a, b)$ there is a chain complex isomorphism

$$
R F H_{*}^{(a, b)}\left(A_{H_{R}-k}\right) \cong R F H_{*}^{(a, b)}\left(A_{H-k}\right) .
$$


Proof. Assuming $R$ is sufficiently large compared to $k$, since all the critical points of $A_{H-k}$ are either points on $\Sigma_{k}$ or parametrizations of periodic orbits of $X_{H}$ lying on $\Sigma_{k}$, we conclude that all the critical points of $A_{H_{R}-k}$ are non-degenerate, and that $\operatorname{Crit}\left(A_{H_{R}-k}\right)=\operatorname{Crit}\left(A_{H-k}\right)$. This shows that the two chain complexes coincide (as groups):

$$
R F_{*}\left(A_{H_{R}-k}\right) \cong R F_{*}\left(A_{H-k}\right) .
$$

Fix an almost complex structure $J \in \mathcal{J}(\omega) \cap B_{\varepsilon_{1}}\left(J_{g}\right)$ and fix a finite interval $(a, b) \subseteq \mathbb{R}$. Let us denote by $\mathcal{M}_{R}$ the set of all maps $u=(x, \eta) \in C^{\infty}\left(\mathbb{R} \times S^{1}, T^{*} M\right) \times C^{\infty}(\mathbb{R}, \mathbb{R})$ that satisfy the Rabinowitz Floer equation $u^{\prime}(s)+\nabla A_{H_{R}-k}(u(s, \cdot))=0$ and have action bounds

$$
A_{H_{R}-k}(u(\mathbb{R})) \subseteq[a, b]
$$

and satisfy

$$
x(\mathbb{R}, \cdot) \in \Lambda_{\alpha} T^{*} M .
$$

We will show how for $R \gg 0$ large enough, one can follow through the proof of Theorem 4.14 and obtain a constant $C_{1}^{\prime}>0$ that serves as a uniform $L^{\infty}$ bound for the $x$-component of elements of $\mathcal{M}_{R}$. Here the key point is that the constant $C_{1}^{\prime}$ is independent of $R$. It will however depend on the interval $(a, b)$. Anyway, this will imply the proposition, as then it is immediate that if we choose $R$ large enough such that

$$
\left\{(q, p) \in T^{*} M:|p| \leq C_{1}^{\prime}\right\} \subseteq\left\{(q, p) \in T^{*} M: H(q, p) \leq R\right\}
$$

then the boundary homomorphisms of the two truncated Rabinowitz Floer complexes necessarily coincide, and hence the two truncated Rabinowitz Floer homologies coincide.

Firstly though let us discuss the $\eta$-component of elements of $\mathcal{M}_{R}$. Nothing in the proof of the bound on the Lagrange multiplier (Theorem 4.11) used anything about the behavior of $H$ at infinity, and thus there exists $R_{0}>0$ such that if $R>R_{0}$, the same constant $C_{0}>0$ obtained in Theorem 4.11 serves as uniform $L^{\infty}$ bound on the $\eta$-component of any element $u=(x, \eta) \in \mathcal{M}_{R}$.

Parts of the argument from the proof of Step 1 of Theorem 4.14 are unchanged for the new Hamiltonian $H_{R}$. Indeed, for any $u=(x, \eta) \in \mathcal{M}_{R}$ if $b_{1}:=\|J\|_{\infty} \sqrt{b-a}$ then as before

$$
\begin{gathered}
\left\|x^{\prime}\right\|_{L^{2}\left(\mathbb{R} \times S^{1}\right)} \leq b_{1} ; \\
\left\|\eta^{\prime}\right\|_{L^{2}(\mathbb{R})} \leq b_{1} .
\end{gathered}
$$

Fix $u=(x, \eta) \in \mathcal{M}_{R}$ and write $x=(q, p)$. Let us introduce the auxiliary smooth function

$$
\begin{gathered}
P_{R}: \mathbb{R} \times S^{1} \rightarrow \mathbb{R} \\
P_{R}(s, t):=\rho_{2 R}\left(|p(s, t)|^{2}\right)=2 \rho_{R}\left(\frac{1}{2}|p(s, t)|^{2}\right) .
\end{gathered}
$$

Note that

$$
X_{H_{R}}(q, p)=\rho_{R}^{\prime}(H(q, p)) X_{H}(q, p) .
$$

Since $\rho_{R}^{\prime} \leq 1$ we see that (4.12) still holds. Let us note that for any $(q, p) \in T^{*} M$ the following two implications hold:

$$
\begin{gathered}
|p|^{2} \geq 2 R+2 R \delta \quad \Rightarrow \quad \rho_{R}^{\prime}\left(\frac{1}{2}|p|^{2}\right)|p|^{2}=0 ; \\
|p|^{2} \leq 4 R-4 R \delta \quad \Rightarrow \quad \rho_{4 R}\left(|p|^{2}\right)=|p|^{2}
\end{gathered}
$$

(where $\delta>0$ is the constant from the definition (6.1) of $\rho$ ). Thus since $\delta<1 / 3$ we always have

$$
\rho_{R}^{\prime}\left(\frac{1}{2}|p|^{2}\right)|p|^{2} \leq \rho_{4 R}\left(\frac{1}{2}|p|^{2}\right) .
$$


In fact, we can improve on this by choosing $R$ sufficiently large. Indeed, suppose

$$
R>R_{1}:=\frac{\|U\|_{\infty}}{1-3 \delta}
$$

Then for any $(q, p) \in T^{*} M$ one has

$$
H(q, p)=R+R \delta \Rightarrow|p|^{2} \leq 4 R-4 R \delta
$$

and hence for $R>R_{1}$ we have

$$
\rho_{R}^{\prime}(H(q, p))|p|^{2} \leq \rho_{4 R}\left(|p|^{2}\right) \quad \text { for every }(q, p) \in T^{*} M .
$$

Thus for $R>R_{1}$,

$$
\left.\left|X_{H_{R}}(x(s, t))\right| \leq b_{0}\left(1+P_{2 R}(s, t)\right)\right) \text { for all }(s, t) \in \mathbb{R} \times S^{1},
$$

where $b_{0}>0$ is defined as before 8 .

In the truncated case, $\eta^{\prime}(s)$ no longer bounds the $L^{2}$ norm of $p(s, \cdot)$, as in (4.16), but instead it bounds the $L^{1}$ norm of $P_{R}(s, \cdot)$. Indeed, since

$$
H_{R}(q, p) \geq \rho_{R}\left(\frac{1}{2}|p|^{2}\right)-\|U\|_{\infty}
$$

for every $(q, p) \in T^{*} M$, we have

$$
\eta^{\prime}(s) \geq \int_{S^{1}} \frac{1}{2} P_{R}(s, t) d t-\left(\|U\|_{\infty}+k\right) .
$$

The same arguments as before successively prove:

- $\left\|P_{R}\right\|_{L^{1}\left(I \times S^{1}\right)} \leq b_{2} \max \left\{|I|,|I|^{1 / 2}\right\}$ for any finite interval $I \subseteq \mathbb{R}$.

- For any $0<\varepsilon \leq 1$ the sets

$$
\begin{aligned}
& \left\{s \in \mathbb{R}:\left\|P_{R}(s, \cdot)\right\|_{L^{1}\left(S^{1}\right)} \leq \frac{b_{2}}{\sqrt{\varepsilon}}\right\} \\
& \left\{s \in \mathbb{R}:\left\|x^{\prime}(s, \cdot)\right\|_{L^{2}\left(S^{1}\right)}^{2} \leq \frac{b_{1}}{\sqrt{\varepsilon}}\right\}
\end{aligned}
$$

are $\varepsilon$-dense in $\mathbb{R}$.

- For any $s \in \mathbb{R},\left\|P_{R}(s, \cdot)\right\|_{L^{1}\left(S^{1}\right)} \leq b_{3}$. We will go through this one in detail: given $s \in \mathbb{R}$, choose $s_{0} \in \mathbb{R}$ such that $\left|s-s_{0}\right| \leq 1$ and $\left\|P_{R}\left(s_{0}, \cdot\right)\right\|_{L^{1}\left(S^{1}\right)} \leq b_{2}$. Without loss of generality

\footnotetext{
${ }^{8}$ In order to aid the reader, throughout this proof the constants $b_{i}$ that appear are the same as the constants $b_{i}$ from the proof of Theorem 4.14 In some cases it is not possible to use exactly the same constant; in this case we denote it by $b_{i}^{\prime}$.
} 
assume $s \geq s_{0}$. Then we have

$$
\begin{aligned}
\left\|P_{R}(s, \cdot)\right\|_{L^{1}\left(S^{1}\right)} & =\left\|P_{R}\left(s_{0}, \cdot\right)\right\|_{L^{1}\left(S^{1}\right)}+\int_{s_{0}}^{s} \frac{d}{d r}\left\|P_{R}(r, \cdot)\right\|_{L^{1}\left(S^{1}\right)} d r \\
& =b_{2}+2 \int_{s_{0}}^{s} \int_{S^{1}} \rho_{R}^{\prime}\left(\frac{1}{2}|p|^{2}\right)\left\langle p(r, t), p^{\prime}(r, t)\right\rangle d t d r \\
& \leq b_{2}+\left.\left.\left.\left.2\left|\int_{s_{0}}^{s} \int_{S^{1}} \rho_{R}^{\prime}\left(\frac{1}{2}|p(r, t)|^{2}\right)\right| p(r, t)\right|^{2} d t d r\right|^{1 / 2}\left|\int_{s_{0}}^{s} \int_{S^{1}}\right| p^{\prime}(r, t)\right|^{2} d t d r\right|^{1 / 2} \\
& \stackrel{(*)}{\leq} b_{2}+2\left|\int_{s_{0}}^{s}\left\|P_{2 R}(r, \cdot)\right\|_{L^{1}\left(S^{1}\right)} d r\right|^{1 / 2}\left\|p^{\prime}\right\|_{L^{2}\left(\left(s_{0}, s\right) \times S^{1}\right)} \\
& \leq b_{2}+2 \sqrt{b_{2}}\left\|x^{\prime}\right\|_{L^{2}\left(\left(s_{0}, s\right) \times S^{1}\right)} \\
& \leq b_{2}+2 \sqrt{b_{2}} b_{1} .
\end{aligned}
$$

Here $(*)$ used (6.3) (note that the assertion from the first bullet point also holds for $P_{2 R}$ !).

Note however that this last assertion does not imply that $\|p(s, \cdot)\|_{L^{2}\left(S^{1}\right)} \leq b_{3}$ for all $s \in \mathbb{R}$, even if $R \gg b_{3}$. In order to prove this we we argue as follows. Let $\vartheta_{R}: \mathbb{R} \rightarrow[0,1]$ denote a smooth function such that agrees with $\rho_{R}$ for $t \geq \delta$, and is equal to $\delta / 2$ for $t \leq 0$, again with $0 \leq \vartheta_{R}^{\prime} \leq 1$. We now introduce another auxiliary smooth function

$$
\begin{gathered}
f_{R}: \mathbb{R} \times S^{1} \rightarrow \mathbb{R} ; \\
f_{R}(s, t):=\vartheta_{R}(|p(s, t)|) .
\end{gathered}
$$

Observe that for any $(s, t) \in \mathbb{R} \times S^{1}$, if $R>R_{2}:=\max \left\{R_{0}, R_{1}\right\}$ then

$$
\begin{aligned}
\dot{f}_{R}(s, t) & \leq|\dot{p}(s, t)| \\
& \leq|\dot{x}(s, t)| \\
& \leq\left|J(t, x(s, t)) \cdot x^{\prime}(s, t)\right|+|\eta(s)|\left|X_{H_{R}}(x(s, t))\right| \\
& \leq\left|J(t, x(s, t)) \cdot x^{\prime}(s, t)\right|+C_{0} b_{0}\left(1+P_{2 R}(s, t)\right) .
\end{aligned}
$$

Thus for $R>R_{2}$,

$$
\left\|\dot{f}_{R}(s, \cdot)\right\|_{L^{1}\left(S^{1}\right)} \leq\|J\|_{\infty}\left\|x^{\prime}(s, \cdot)\right\|_{L^{2}\left(S^{1}\right)}+C_{0} b_{0}\left(1+\left\|P_{2 R}(s, \cdot)\right\|_{L^{1}\left(S^{1}\right)}\right) .
$$

Now observe that

$$
\left\|f_{R}(s, \cdot)\right\|_{L^{1}\left(S^{1}\right)} \leq 1+\left\|P_{R}(s, \cdot)\right\|_{L^{1}\left(S^{1}\right)}
$$

(since at any point $t \in S^{1}$, either $f_{R}(s, t) \leq 1$ or $f_{R}(s, t) \leq P_{R}(s, t)$ ). It now follows from (4.19) and (4.23) that if

$$
b_{4}^{\prime}:=N\left(1+b_{3}\right)+N C_{0} b_{0}\left(1+b_{3}^{2}\right),
$$

where $N>0$ is defined as in (4.22) then for any $0<\varepsilon \leq 1$ the subset

is $\varepsilon$-dense. Let

$$
\left\{s \in \mathbb{R}:\left\|f_{R}(s, \cdot)\right\|_{L^{\infty}\left(S^{1}\right)} \leq b_{4}^{\prime}+\frac{b_{5}}{\sqrt{\varepsilon}}\right\}
$$

$$
R_{3}:=\max \left\{R_{2}, \frac{b_{4}^{\prime}+b_{5}}{1-\delta}\right\} \text {. }
$$

Then for $R>R_{3}$, we know that the set $\left\{s \in \mathbb{R}:\|p(s, \cdot)\|_{L^{\infty}\left(S^{1}\right)} \leq b_{4}^{\prime}+b_{5}\right\}$ is 1-dense in $\mathbb{R}$, and then arguing as before, we discover that there exists a constant $b_{3}^{\prime}>0$ such that

$$
\|p(s, \cdot)\|_{L^{2}\left(S^{1}\right)} \leq b_{3}^{\prime} \text { for all } s \in \mathbb{R} .
$$


Next, since $\rho_{R}^{\prime} \leq 1$, the same argument as before shows that for all $s_{0}<s_{1}$ we have

$$
\|\nabla p\|_{L^{2}\left(\left(s_{0}, s_{1}\right) \times S^{1}\right)}^{2} \leq b_{6}\left(\left|s_{1}-s_{0}\right|+b_{1}^{2}\right)+b_{6}\|p\|_{L^{4}\left(\left(s_{0}, s_{1}\right) \times S^{1}\right)}^{4} .
$$

Now let us choose $\varepsilon=\varepsilon_{*}$ where $\varepsilon_{*}=\varepsilon_{*}\left(b_{3}^{\prime}, b_{6}\right)$ is the constant from Lemma4.15, and choose

$$
R>R(a, b):=\max \left\{R_{3}, b_{4}^{\prime}+\frac{b_{5}}{\sqrt{\varepsilon_{*}}}\right\} .
$$

Then for $R>R(a, b)$ the set

$$
\left\{s \in \mathbb{R}:\|p(s, \cdot)\|_{L^{\infty}\left(S^{1}\right)} \leq b_{4}^{\prime}+\frac{b_{5}}{\sqrt{\varepsilon_{*}}}\right\}
$$

is $\varepsilon_{*}$-dense in $\mathbb{R}$. Thus by (6.5), (6.6) and (6.7), Lemma 4.15 implies that there exists a constant $b_{7}^{\prime}=\Gamma\left(b_{1}, b_{3}^{\prime}, b_{6}, b_{4}^{\prime}+b_{5} / \sqrt{\varepsilon_{*}}\right)$ such that for $R>R(a, b)$ the following holds: for any finite interval $I \subseteq \mathbb{R}$ we have

$$
\|\nabla p\|_{L^{2}\left(I \times S^{1}\right)} \leq b_{7}^{\prime}\left(1+|I|^{1 / 2}\right) .
$$

In other words, for $R>R(a, b)$, Step 1 of Theorem 4.14 goes through, and the constant $K^{\prime}>0$ that we obtain is independent of $R$. Moving onto Step 2, we note that the proof of Step 2 used nothing about the Hamiltonian other than the fact that Step 1 holds, and that (4.12) holds. Thus the proof goes through immediately for the Hamiltonian $H_{R}$ with $R>R(a, b)$. Moreover the constant $C_{1}^{\prime}>0$ that Step 2 produced depended only on the constants $K^{\prime}$ and $b_{0}$. Thus we have proved that there exists a constant $C_{1}^{\prime}>0$ such that if $R>R(a, b)$ and $u=(x, \eta) \in \mathcal{M}_{R}$ then

$$
\|x\|_{L^{\infty}\left(\mathbb{R} \times S^{1}\right)} \leq C_{1}^{\prime} .
$$

By the remarks at the beginning of the proof this implies the result.

Let us denote by $R F H_{*}\left(\Sigma_{k}, T^{*} M\right)$ the Rabinowitz Floer homology of the hypersurface $\Sigma_{k}$ as definedg] in [20]. We now prove that $R F H_{*}\left(\Sigma_{k}, T^{*} M\right) \cong R F H_{*}\left(A_{H-k}\right)$. It is sufficient to prove this when $(g, \sigma, U, k) \in \mathcal{O}_{\text {reg }}$ (cf. Remark 4.10). Since the Hamiltonian $H_{R}$ is constant outside of a compact set, using the invariance result [20, Theorem 1.1] we conclude that we can compute $R F H_{*}\left(\Sigma_{k}, T^{*} M\right)$ using $10 H_{R}$, and thus for $R>R(a, b)$ :

$$
R F H_{*}^{(a, b)}\left(A_{H_{R}-k}\right) \cong R F H_{*}^{(a, b)}\left(\Sigma_{k}, T^{*} M\right) .
$$

Then using [17, Theorem A], which tells ${ }^{11}$ us that we can determine both the Rabinowitz Floer homologies $R F H_{*}\left(A_{H-k}\right)$ and $R F H_{*}\left(\Sigma_{k}, T^{*} M\right)$ from the truncated Rabinowitz Floer homologies via:

We conclude that

$$
\begin{aligned}
& R F H_{*}\left(A_{H-k}\right) \cong \underset{a \downarrow-\infty b \uparrow \infty}{\lim } \lim _{\underset{b}{ }} R F H_{*}^{(a, b)}\left(A_{H-k}\right) ; \\
& R F H_{*}\left(\Sigma_{k}, T^{*} M\right) \cong \underset{a \downarrow-\infty b \uparrow \infty}{\lim _{a \downarrow}} \lim _{\leftarrow} R F H_{*}^{(a, b)}\left(\Sigma_{k}, T^{*} M\right) .
\end{aligned}
$$

$$
R F H_{*}\left(A_{H-k}\right) \cong R F H_{*}\left(\Sigma_{k}, T^{*} M\right) .
$$

We can now prove the main result of this paper. In the proof below for clarity we will continue to write $R F H_{*}\left(A_{H-k}\right)$ for the Rabinowitz Floer homology as defined in this paper, and $R F H_{*}\left(\Sigma_{k}, T^{*} M\right)$

\footnotetext{
${ }^{9}$ Technically the Rabinowitz Floer homology $\operatorname{RF} H_{*}\left(\Sigma_{k}, T^{*} M\right)$ as defined in [20] is only defined for contractible loops. If however one uses the observation that $\omega$ is symplectically atoroidal then the construction in [20] allows one to define Rabinowitz Floer homology $R F H_{*}\left(\Sigma_{k}, T^{*} M\right)$ for any free homotopy class; see Remark 1.4

${ }^{10}$ Here we are implicitly using the last sentence of Remark 6.1

${ }^{11}$ This is the only time in the entire paper where it is absolutely essential that we used field coefficients for the Rabinowitz Floer homology rather than, say, $\mathbb{Z}$-coefficients.
} 
for the Rabinowitz Floer homology defined in [20], despite the fact that we have just proved the two are isomorphic.

\section{Proof. (of Theorem 1.1)}

We are given a closed weakly exact 2-form $\sigma \in \Omega_{\text {we }}^{2}(M)$ and a potential $U \in C^{\infty}(M, \mathbb{R})$, together with a value $k \in \mathbb{R}$ such that $k>c(g, \sigma, U)$. Put $H:=H_{g}+\pi^{*} U$ and $\Sigma_{k}:=H^{-1}(k)$. We will compute $R F H_{*}\left(\Sigma_{k}, T^{*} M\right)$. By Remark 4.10 we may assume that $(g, \sigma, U, k) \in \mathcal{O}_{\text {reg. }}$. We begin by choosing $r>0$ such that $\|r \sigma\|_{\infty}$ is sufficiently small such that the conclusion of Theorem 5.1 holds. Let us temporarily write $R F H_{*}\left(\Sigma_{k}, T^{*} M ; \omega\right)$ to indicate which symplectic form we are working with. Then

$$
R F H_{*}\left(\Sigma_{k}, T^{*} M ; \omega\right) \cong R F H_{*}\left(\Sigma_{k}, T^{*} M ; r \omega\right) .
$$

To see we argue as follows. If $F \in C_{c}^{\infty}\left(T^{*} M, \mathbb{R}\right)$ is a defining Hamiltonian for $\left(H, \Sigma_{k}, \omega\right)$ in the sense of [20], that is, $F$ is a compactly supported Hamiltonian such that $\Sigma_{k}=F^{-1}(0)$, and $\left.X_{F}\right|_{\Sigma_{k}}=$ $\left.X_{H}\right|_{\Sigma_{k}}$, then since $X_{F}^{\omega}=X_{r F}^{r \omega}$ (here $X_{F}^{\omega}$ denotes the symplectic gradient of $F$ with respect to $\omega$, etc.), the Hamiltonian $r F$ is a defining Hamiltonian for $\left(H, \Sigma_{k}, r \omega\right)$. Next, there is a natural identification between flow lines of the two Rabinowitz action functionals $A_{F}$ and $A_{r F}:$ if $u(s, t)=(x(s, t), \eta(s))$ satisfies $u^{\prime}(s)+\nabla A_{F}(u(s))=0$ then $u_{r}(s, t):=(x(s, t), \eta(r s))$ satisfies $u_{r}^{\prime}(s)+\nabla A_{r F}\left(u_{r}(s)\right)=0$, and vice versa. This identification defines a chain isomorphism between the two chain complexes.

Set $\omega_{r}:=\omega_{0}+r \pi^{*} \sigma$ so that $\omega=\omega_{1}$. Next we claim

$$
R F H_{*}\left(\Sigma_{k}, T^{*} M ; r \omega\right) \cong R F H_{*}\left(H_{r}^{-1}\left(r^{2} k\right), T^{*} M ; \omega_{r}\right),
$$

where $H_{r}(q, p):=H_{g}+r^{2} \pi^{*} U$ (note that the latter is well defined, as by Lemma2.2 we have $k>$ $c(g, \sigma, U)$ if and only if $\left.r^{2} k>c\left(g, r \sigma, r^{2} U\right)\right)$. Indeed, the exact symplectomorphism $\varphi_{r}: T^{*} M \rightarrow$ $T^{*} M$ defined by

$$
\varphi_{r}(q, p):=(q, r p)
$$

satisfies

$$
\begin{gathered}
\varphi_{r}^{*} \omega_{r}=r \omega ; \\
\varphi_{r}^{*} H_{r}=r^{2} H,
\end{gathered}
$$

and hence $\varphi_{r}\left(\Sigma_{k}\right)=H_{r}^{-1}\left(r^{2} k\right)$. The Rabinowitz Floer homology of [20] is invariant under such symplectomorphisms, and hence the claim follows. Next, by (6.8) we have

$$
R F H_{*}\left(H_{r}^{-1}\left(r^{2} k\right), T^{*} M ; \omega_{r}\right) \cong R F H_{*}\left(A_{H_{r}-r^{2} k} ; \omega_{r}\right),
$$

and finally by our choice of $r$ we can compute $R F H_{*}\left(A_{H_{r}-r^{2} k} ; \omega_{r}\right)$ via Theorem 5.1

\subsection{Leaf-wise intersections.}

We conclude this paper by showing how the fact that $R F H_{*}\left(\Sigma_{k}, T^{*} M\right)$ is non-zero for $k>c(g, \sigma, U)$ implies the existence of leaf-wise intersections, following [6, 5]. Throughout this section assume that $(g, \sigma, U, k) \in \mathcal{O}$ (in general we do not need to assume that $(g, \sigma, U, k) \in \mathcal{O}_{\text {reg }}$, although this will be needed to get infinitely many leaf-wise intersections), and put $H:=H_{g}+\pi^{*} U$ and $\Sigma_{k}:=H^{-1}(k)$.

The hypersurface $\Sigma_{k}$ is foliated by the leaves $\left\{\mathcal{L}_{x}: x \in \Sigma_{k}\right\}$, where

$$
\mathcal{L}_{x}:=\left\{\phi_{t}^{H}(x): t \in \mathbb{R}\right\} .
$$

Let $\operatorname{Ham}_{c}\left(T^{*} M, \omega\right)$ denote the set of compactly supported 1-periodic Hamiltonian diffeomorphisms of the symplectic manifold $\left(T^{*} M, \omega\right)$, that is

$$
\operatorname{Ham}_{c}\left(T^{*} M, \omega\right):=\left\{\phi_{1}^{F}: F \in C_{c}^{\infty}\left(S^{1} \times T^{*} M, \mathbb{R}\right)\right\},
$$

where $\phi_{t}^{F}$ is the flow of $X_{F}$; the latter being the time-dependent symplectic gradient of $F$ with respect to $\omega$. Given $\psi \in \operatorname{Ham}_{c}\left(T^{*} M, \omega\right)$, a point $x \in \Sigma_{k}$ is called a leaf-wise intersection point for $\psi$ if 
$\psi(x) \in \mathcal{L}_{x}$.

In order to explain the beautiful idea of Albers and Frauenfelder that links Rabinowitz Floer homology to leaf-wise intersections, we will need some preliminary definitions. First let us define

$$
\mathcal{X}:=\left\{\chi \in C^{\infty}\left(S^{1}, \mathbb{R}\right): \int_{S^{1}} \chi(t) d t=1, \operatorname{supp}(\chi) \subseteq(0,1 / 2)\right\} .
$$

We will say that a time-dependent Hamiltonian $G: S^{1} \times T^{*} M \rightarrow \mathbb{R}$ is $H$-admissible if:

(1) $G(t, x)=\chi(t) G_{0}(x)$ for some $\chi \in \mathcal{X}$ and some compactly supported $G_{0} \in C_{c}^{\infty}\left(T^{*} M, \mathbb{R}\right)$.

(2) $G_{0}^{-1}(0)=\Sigma_{k}$.

(3) It holds that $X_{G_{0}}\left|\Sigma_{k}=X_{H}\right|_{\Sigma_{k}}$.

Let us write $\mathcal{H}(H)$ for the set of $H$-admissible Hamiltonians. Finally set

$$
\mathcal{F}:=\left\{F \in C_{c}^{\infty}\left(S^{1} \times T^{*} M, \mathbb{R}\right): F(t, \cdot) \equiv 0 \text { for } t \in[1 / 2,1]\right\} .
$$

It is easy to see that $\mathcal{F}$ generates $\operatorname{Ham}_{c}\left(T^{*} M, \omega\right)$ in the sense that given any $\psi \in \operatorname{Ham}_{c}\left(T^{*} M, \omega\right)$, there exists $F \in \mathcal{F}$ such that $\psi=\phi_{1}^{F}$ (see for example [6, Lemma 2.3]).

Let us call a pair $(G, F) \in \mathcal{H}(H) \times \mathcal{F}$ a Moser pair for $\Sigma_{k}$. Given a Moser pair $(G, F)$ for $\Sigma_{k}$, define the perturbed Rabinowitz action functional $A_{G-k}^{F}: \Lambda T^{*} M \times \mathbb{R} \rightarrow \mathbb{R}$ by

$$
A_{G-k}^{F}(x, \eta):=\int_{C} \bar{x}^{*} \omega-\eta \int_{S^{1}} G(t, x) d t-\int_{S^{1}} F(t, x) d t
$$

(where $\bar{x}$ and $C$ are defined as before). A short calculation shows that

$\operatorname{Crit}\left(A_{G-k}^{F}\right)=\left\{(x, \eta) \in C^{\infty}\left(S^{1}, T^{*} M\right) \times \mathbb{R}: \dot{x}=\eta \chi(t) X_{G_{0}}(x)+X_{F}(t, x), \int_{S^{1}} \chi(t) G_{0}(x) d t=0\right\}$.

The key observation of Albers and Frauenfelder that makes the whole approach work is the following lemma [6, Proposition 2.4].

6.3. LemmA. Suppose $(x, \eta) \in \operatorname{Crit}\left(A_{G-k}^{F}\right)$. Then if $\psi=\phi_{1}^{F}$ and $y:=x(1 / 2) \in \Sigma$ then $\psi(y)=\mathcal{L}_{y}$, that is, $y$ is a leaf-wise intersection point for $\psi$ in $\Sigma_{k}$.

Proof. For $t \in[0,1 / 2]$ we have $G_{0}(x(t))$ constant, since $X_{F}(t, \cdot)=0$, and hence $x(t) \in \Sigma_{k}$ for $t \in[0,1 / 2]$. For $t \in[1 / 2,1], x(t)$ satisfies $\dot{x}(t)=X_{F}(t, x(t))$ and hence $x(1)=\psi(x(1 / 2))$. Thus if $y:=x(1 / 2)$ then $y$ and $\psi(y)$ both lie in $\Sigma_{k}$. Moreover since on $[0,1 / 2]$ we have $\dot{x}(t)=\eta \chi(t) G_{0}(x(t))$ we have $\psi(y)=x(0) \in \mathcal{L}_{y}$. The proof is complete.

Let us say that a leaf-wise intersection point $y \in \Sigma_{k}$ for $\psi \in \operatorname{Ham}_{c}\left(T^{*} M, \omega\right)$ is a periodic leaf-wise intersection point for $\psi$ if the leaf $\mathcal{L}_{x}$ is a closed orbit of $\phi_{t}^{H}$. It is clear from the proof above that the map $\operatorname{Crit}\left(A_{G-k}^{F}\right) \rightarrow\left\{\right.$ leaf-wise intersection points for $\left.\phi_{1}^{F}\right\}$ is injective if there do not exist any periodic leaf-wise intersection points for $\psi$.

We will now state the two analytic results about the perturbed twisted Rabinowitz action functional $A_{G-k}^{F}$ that allow one to do Rabinowitz Floer homology with it. The proof of the first theorem is essentially identical to [6, Theorem 2.14] and [5, Theorem 3.3].

6.4. TheOREM. Fix $G \in \mathcal{H}(H)$. Let $\mathcal{F}_{\text {reg }}(G) \subseteq \mathcal{F}$ denote the set of functions $F$ such that $A_{G-k}^{F}$ is a Morse function. Then $\mathcal{F}_{\text {reg }}(G)$ is residual in $\mathcal{F}$. Moreover if $(g, \sigma, U, k) \in \mathcal{O}_{\text {reg }}$ then the set $\widetilde{\mathcal{F}}_{\text {reg }}(G) \subseteq \mathcal{F}_{\text {reg }}(G)$ consisting of those functions $F \in \mathcal{F}_{\text {reg }}(G)$ for which there do not exist any periodic leaf-wise intersection points for $\phi_{1}^{F}$ in $\Sigma_{k}$, is also residual in $\mathcal{F}$. 
The following result is proved exactly as in [6, Theorem 2.9], aside from the fact that one needs to use the modifications already present in the proof of Theorem 4.11 above to deal with the fact that $\Sigma_{k}$ is only of virtual restricted contact type.

6.5. TheOREM. Let $-\infty<a<b<\infty$ and $\alpha \in\left[S^{1}, M\right]$, and let $\mathcal{M}$ denote the set of gradient flow lines $u \in C^{\infty}\left(\mathbb{R} \times S^{1}, T^{*} M\right) \times C^{\infty}(\mathbb{R}, \mathbb{R})$ of $A_{G-k}^{F}$ (with respect to a suitable compatible almost complex structure) such that $A_{G-k}^{F}(u(\mathbb{R})) \subseteq[a, b]$ and $x(\mathbb{R}, \cdot) \subseteq \Lambda_{\alpha} T^{*} M$. Then $\mathcal{M}$ is precompact in $C^{\infty}\left(\mathbb{R} \times S^{1}, T^{*} M\right) \times C^{\infty}\left(\mathbb{R} \times S^{1}, \mathbb{R}\right)$, where this space is given the $C_{\mathrm{loc}}^{\infty}$ topology.

Using the previous two theorems (see [6, Section 2] for the full details), if $F \in \mathcal{F}_{\text {reg }}(G)$ one can define the Rabinowitz Floer homology $R F H_{*}\left(A_{G-k}^{F}\right)$ of the perturbed Rabinowitz action functional $A_{G-k}^{F}$, and show moreover that

$$
R F H_{*}\left(A_{G-k}^{F}\right) \cong R F H_{*}\left(A_{G-k}^{F=0}\right) \stackrel{\text { def }}{=} R F H_{*}\left(\Sigma_{k}, T^{*} M\right) .
$$

In particular, given $F \in \mathcal{F}_{\text {reg }}(G)$ we have the following corollary of Theorem 5.1.

6.6. Corollary. For degrees $* \neq 0,1$,

$$
R F H_{*}\left(A_{G-k}^{F}\right) \cong\left\{\begin{array}{l}
H_{*}\left(\Lambda M ; \mathbb{Z}_{2}\right) \\
H^{1-*}\left(\Lambda M ; \mathbb{Z}_{2}\right) .
\end{array}\right.
$$

Using the corollary it is easy to complete the proof of Theorem 1.6 from the introduction.

\section{Proof. (of Theorem 1.6)}

First we show that any $\psi \in \operatorname{Ham}_{c}\left(T^{*} M, \omega\right)$ has a leaf-wise intersection point. Indeed, if not then we can find $F \in \mathcal{F}$ and $G \in \mathcal{H}(H)$ such that $\psi=\phi_{1}^{F}$ and $\operatorname{Crit}\left(A_{G-k}^{F}\right)=\emptyset$ (see for instance [16, p279-280]). In this case $A_{G-k}^{F}$ is trivially Morse, and hence $F \in \mathcal{F}_{\text {reg }}(G)$. But if $\operatorname{Crit}\left(A_{G-k}^{F}\right)=\emptyset$ then $R F H_{*}\left(A_{G-k}^{F}\right)=0$, a contradiction.

Suppose now that $\operatorname{dim} H_{*}\left(\Lambda M ; \mathbb{Z}_{2}\right)=\infty$ and $(g, \sigma, U, k) \in \mathcal{O}_{\text {reg. }}$. Then for a generic $\psi \in$ $\operatorname{Ham}_{c}\left(T^{*} M, \omega\right)$, we can write $\psi=\phi_{1}^{F}$ for some $F \in \widetilde{\mathcal{F}}_{\text {reg }}(G)$. In this case the previous corollary combined with Lemma 6.3 implies the existence of infinitely many leaf-wise intersection points for $\psi$ in $\Sigma_{k}$.

\section{REFERENCES}

1. A. Abbondandolo and P. Majer, Lectures on the Morse complex for infinite dimensional manifolds, Morse Theoretic Methods in Nonlinear Analysis and Symplectic Topology (P Biran, O. Cornea, and F. Lalonde, eds.), Nato Science Series II: Mathematics, Physics and Chemistry, vol. 217, Springer-Verlag, 2006, pp. 1-74.

2. A. Abbondandolo, A. Portaluri, and M. Schwarz, The homology of path spaces and Floer homology with conormal boundary conditions, J. Fixed Point Theory Appl. 4 (2008), no. 2, 263-293.

3. A. Abbondandolo and M. Schwarz, On the Floer homology of cotangent bundles, Comm. Pure Appl. Math. 59 (2006), 254-316.

4. __ Estimates and computations in Rabinowitz-Floer homology, Jour. Topology Analysis 1 (2009), no. 4, $307-405$.

5. P. Albers and U. Frauenfelder, Infinitely many leaf-wise intersection points on cotangent bundles, arXiv:0812.4426 (2009).

6. __ Leaf-wise intersections and Rabinowitz Floer homology, Jour. Topology Analysis 2 (2010), no. 1, 77-98.

7. __ Rabinowitz Floer homology: A Survey, arXiv:1001.4272 (2010).

8. A A Remark on a Theorem by Ekeland-Hofer, to appear in Isr. J. Math. (2010).

9. Spectral Invariants in Rabinowitz Floer homology and Global Hamiltonian perturbations, J. Modern Dynamics 4 (2010), 329-357.

10. V. I. Arnold and A. B. Givental, Symplectic Geometry, Dynamical Systems, Encyclopedia of Mathematical Sciences, vol. IV, Springer-Verlag, 1990.

11. M. Audin and J. Lafontaine (eds.), Holomorphic curves in symplectic geometry, Progress Math., vol. 117, Birkhäuser Verlag, 1994. 
12. Y. Bae and U. Frauenfelder, Continuation homomorphism in Rabinowitz Floer homology for symplectic deformations, arXiv:101.1649 (2010).

13. A. Bahri, Critical points at infinity in some variational problems, Pitman Research Notes in Mathematics, vol. 182, Longman, 1989.

14. F. Bourgeois and A. Oancea, The Gysin exact sequence for $S^{1}$-equivariant symplectic homology, arxiv:0909.4526 (2009).

15. K. Burns and G. P. Paternain, Anosov magnetic flows, critical values and topological entropy, Nonlinearity 15 (2002), 281-314.

16. K. Cieliebak and U. Frauenfelder, A Floer homology for exact contact embeddings, Pacific J. Math. 239 (2009), no. 2, 216-251.

17. __ Morse homology on noncompact manifolds, Preprint (2009).

18. Corrigendum to "A Floer homology for exact contact embeddings", (2010).

19. K. Cieliebak, U. Frauenfelder, and A. Oancea, Rabinowitz Floer homology and symplectic homology, Ann. Sci. École Norm. Sup 43 (2010), no. 6.

20. K. Cieliebak, U. Frauenfelder, and G. P. Paternain, Symplectic topology of Mañé's critical value, Geometry and Topology 14 (2010), 1765-1870.

21. K. Cieliebak, V. Ginzburg, and E. Kerman, Symplectic homology and periodic orbits near symplectic submanifolds, Comment. Math. Helv. 74 (2004), 554-581.

22. G. Contreras, The Palais-Smale condition on contact type energy levels for convex Lagrangian systems, Calc. Var. Partial Differential Equations 27 (2006), no. 3, 321-395.

23. G. Contreras and R. Iturriaga, Global Minimizers of Autonomous Lagrangians, Colloqio Brasileiro de Matematica, vol. 22, IMPA, Rio de Janeiro, 1999.

24. G. Contreras, R. Iturriaga, G. P. Paternain, and M. Paternain, The Palais-Smale Condition and Mañé's Critical Values, Ann. Henri Poincaré 1 (2000), 655-684.

25. J. J. Duistermaat, On the Morse Index in Variational Calculus, Adv. Math. 21 (1976), 173-195.

26. A. Fathi and E. Maderna, Weak KAM theorem on non compact manifolds, Nonlinear Diff. Eq. Appl. 14 (2007), 1-27.

27. A. Floer, H. Hofer, and D. Salamon, Transversality in elliptic Morse theory for the symplectic action, Duke Math. J. 80 (1996), 252-292.

28. U. Frauenfelder, The Arnold-Givental conjecture and moment Floer homology, Int. Math. Res. Not. 42 (2004), 21792269.

29. V. Ginzburg, On closed trajectories of a charge in a magnetic field. An application of symplectic geometry, Contact and symplectic geometry (Cambridge, 1994) (C. B. Thomas, ed.), Publications of the Newton Institute, vol. 8, Cambridge University Press, 1996, pp. 131-148.

30. M. Gromov, Kähler hyperbolicity and $L^{2}$-Hodge theory, J. Differential Geom. 33 (1991), 263-292.

31. H. Hofer and E. Zehnder, Symplectic invariants and Hamiltonian dynamics, Birkhäuser Verlag, 1994.

32. J. Kang, Generalized Rabinowitz Floer homology and coisotropic intersections, arxiv:1003.1009 (2010).

33. W. Klingenberg and F. Takens, Generic properties of geodesic flows, Math. Ann. 197 (1972), 323-334.

34. G. Lu, The Weinstein conjecture on some symplectic manifolds containing the holomorphic spheres, Kyushu J. Math. 50 (1996), 331-351.

35. R. Mañé, Lagrangian flows: the dynamics of globally minimizing orbits, International Congress on Dynamical Systems in Montevideo (a tribute to Ricardo Mañé) (J. Lewowicz F. Ledrappier and S. Newhouse, eds.), Pitman Research Notes in Math., vol. 362, Longman, 1996, pp. 120-131.

36. L. Macarini and G. P. Paternain, On the stability of Mañé critical hypersurfaces, to appear in Calc. Var. Partial Differential Equations (2010).

37. J. Mawhim and M. Willem, Critical Point Theory and Hamiltonian Systems, Appl. Math. Sci., vol. 74, Springer-Verlag, 1989.

38. W. J. Merry, Closed orbits of a charge in a weakly exact magnetic field, Pacific J. Math. 247 (2010), 189-212.

39. L_ Lagrangian Rabinowitz. Floer homology of twisted cotangent bundles, arXiv:1010.4190 (2010).

40. Ph.D. thesis, University of Cambridge, 2011.

41. W. J. Merry and G. P. Paternain, Index computations in Rabinowitz Floer homology, arXiv:1009.3870, to appear in J. Fixed Point Theory Appl. (2010).

42. C. J. Niche, Non-contractible periodic orbits of Hamiltonian flows on twisted cotangent bundles, Discrete Contin. Dyn. Syst. 14 (2006), no. 4, 617-630.

43. G. P. Paternain, Magnetic Rigidity of Horocycle Flows, Pacific J. Math. 225 (2006), 301-323.

44. G. P. Paternain and M. Paternain, Critical values of autonomous Lagrangians, Comment. Math. Helv. 72 (1997), 481499.

45. P. H. Rabinowitz, Periodic solutions of Hamiltonian systems, Comm. Pure. Appl. Math. 31 (1978), 157-184.

46. J. Robbin and D. Salamon, The Maslov index for paths, Topology 32 (1993), 827-844. 
47. P. Seidel, Graded Lagrangian Submanifolds, Bull. Soc. Math. France 128 (2000), 103-149.

48. J. Weber, Perturbed closed geodesics are periodic orbits: Index and transversality, Math. Z. 241 (2002), 45-81.

Department of Pure Mathematics and Mathematical Statistics, University of Cambridge, CamBRIDGE CB3 0WB, ENGLAND

E-mail address: w. merry@dpmms.cam.ac.uk 